\title{
Domains of Attraction of Generic $\omega$-Limit Sets for Strongly Monotone Semiflows
}

\author{
P. TAKÁČ
}

\begin{abstract}
Asymptotic behavior of a strongly increasing semiflow $\Phi$ in a strongly ordered metrizable topological space $X$ is investigated in terms of the $\omega$-limit set $\omega(x)$ of a generic point $\boldsymbol{I} \in$ whose positive semiorbit $\mathcal{O}^{+}(x)$ is assumed to be relatively compact. The domain of attraction of the $\omega$-limit set of a generic order $\omega$-stable point is determined. If $X$ is an open and order-convex subset of a separable strongly ordered Banach space $V$, it is proved that "almost all" points $I \in X$ are order $\omega$-stable, whereas the remaining $\omega$-unstable points are contained in the union of at most countably many Lipschitz manifolds of codimension one in $V$. If $\Phi$ admits a strongly pasitive, compact linearization about its equilibria, then $\omega(x)$ is a single equilibrium for every order $\omega$-stable point $x \in X$.
\end{abstract}

Key words: convergence to equilibrium, invariant order decomposition and resolution, lower and upper $\omega$-limit set6, cooperative system of ordinary differential equations, parabolic partial differential equation

AMS subject classifications: primary 88F12, secondary $70 \mathrm{K20}$

\section{Introduction}

The primary goal of this paper is to investigate the asymptotic behavior and the domain of attraction of a generic $\omega$-limit set for a continuous-time semiflow $\Phi \equiv\left\{\Phi_{t}: t \in R_{+}^{1}\right\}$ where $\Phi_{\imath}: X \longrightarrow X$, for every $t>0$, is a strongly increasing continuous mapping in a strongly ordered, metrizable topological space $X$. For instance, $X$ can be an open subset of a strongly ordered Banach space $V$, i.e., $V$ is a real Banach space with an order relation " $\leq$ " whose positive cone $V_{+}=\{x \in V: x \geq 0\}$ has nonempty interior Int $\left(V_{+}\right)$. We write $x \ll y$ in $V$ iff $y-x \in \operatorname{Lnt}\left(V_{+}\right)$, whereas $x<y$ in $V$ means $x \leq y$ and $x \neq y$. Then $T: X \longrightarrow X$ is called strongly increasing iff $x<y$ in $X \subset V$ implies $T x \ll T y$.

To describe the asymptotic behavior of our semillow $\Phi$ we study the $\omega$-limit set $\omega(x)$ of a generic point $x \in X$ whose positive semiorbit (orbit, for short) $\mathcal{O}^{+}(x)=\left\{\Phi_{1} x: t \in R_{+}^{1}\right\}$ is assumed to be relatively compact in $X$. Here $\omega(x)$ is the set of all limit points in $X$ of $\Phi_{\imath} x$ as $t \longrightarrow \infty$. Asymptotic behavior and domains of attraction of $\boldsymbol{\omega}$-limit sets are closely connected through stability properties of $x \in X$ expressed in terms of the $\omega$-limit sets $\omega(y)$ for $y \in X$ near $x, y \lesseqgtr x$, which we call $w$-stability. To state this stability classification we assume that $\Phi$ is $\omega$-compact in every simply ordered, compact $\operatorname{arc} J \subset X$, i.e., $\mathcal{O}^{+}(x)$ is

\footnotetext{
- This research was supported in part by the National Science Foundation under the grant DMS-8802646.
} 
relatively compact for every $x \in J$, and also $\underset{x \in J}{\cup} \omega(x)$ is relatively compact in $X$. Given any $x \in X$, we first define the lower and upper $\omega$-limit sets of $x$, respectively, by

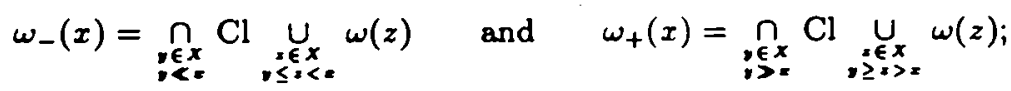

their properties are described in Proposition 3.4 through Corollary 3.7. Here "Cl" denotes the closure in $X$.

A point $x \in X$ is called lower $\omega$-stable (symbolically $x \in S_{-}$) if $\omega_{-}(x)=\omega(x)$; otherwise $x$ is lower $\omega$-unstable $\left(x \in \mathcal{U}_{-}\right)$. A point $x \in \mathcal{S}_{-}$is called lower asymptotically $\omega$-stable $\left(x \in \mathcal{A}_{-}\right)$if $\omega(y)=\omega(x)$ for some $y \in X, y<x$. The upper $\omega$-stability classification of $x \in X$ is defined analogously with $\omega_{+}(x)$ in place of $\omega_{-}(x)$. We set $\mathcal{S}=\mathcal{S}_{-} \cap \mathcal{S}_{+}$ $\mathcal{S}_{1 / 2}=\mathcal{S}_{-} \cup \mathcal{S}_{+}, \mathcal{U}=\mathcal{U}_{-} \cup \mathcal{U}_{+}, \mathcal{U}_{2}=\mathcal{U}_{-} \cap \mathcal{U}_{+}, \mathcal{A}=\mathcal{A}_{-} \cap \mathcal{A}_{+}$and $\mathcal{A}_{1 / 2}=\mathcal{A}_{-} \cup \mathcal{A}_{+}$.

Our most important result describing the asymptotic behavior of $\Phi$, Theorem 5.1 sup plemented by Proposition 4.4 and Corollary 4.5, shows that "almost all" points $x \in X$ are order $\omega$-stable, i.e., $x \in \mathcal{S}$, and either $\omega(x) \subset \mathcal{S}_{1 / 2}$ is a single equilibrium, or else $\omega(x) \subset \mathcal{U}_{2}$ is a set of equilibria with $x \in \mathcal{A}_{1 / 2}$. We denote by $\mathcal{E}=\left\{x \in X: \Phi_{t} x=x\right.$ for all $\left.t \in \mathbf{R}_{+}^{1}\right\}$ the set of all equilibria. The main part of this result, namely $\omega(x) \subset \mathcal{E}$ for "almost all" $x \in X$ was proved already by Hirsch [20, Thm. 7.5 and 7.8] and later in a more general space setting by Smith \& Thieme [41, Thm. 3.3]. For a very common class of semiflows associated with autonomous evolution equations, e.g., irreducible cooperative systems of ordinary differential equations or semilinear parabolic partial differential equations, it was shown by Polácik [32, Thm. 1] and Smith \& Thieme [42, Prop. 2.5] that $\omega(x)$ is a single equilibrium for every $x \in S$, cf. also our Theorem 5.2. Our approach has an interesting addition to their results, namely, description of $\mathcal{U}_{-}$and $\mathcal{U}_{+}$as the unions of at most countably many Lipschitz manifolds, thus showing that Smale's example [37] is; in fact, rather general. Our study of $\omega$-unstable points was inspired by Hirsch [21, Thm. 1.1 and Prop. 2.6]. Furthermore, $\mathcal{U}_{-} \subset \partial_{+} \mathcal{A}$ and $\mathcal{U}_{+} \subset \partial_{-} \mathcal{A}$, where $\partial_{-}$and $\partial_{+}$denote the lower and upper boundaries. resp., cf. Section 4. In particular, a strongly increasing continuous-time dynamical system. $(X, \Phi)$ cannot be "very chaotic"; all "chaos" must occur inside $\mathcal{U}_{2}$, the set of all $\omega$-biurstable points. To state this and other results precisely we will assume, for simplicity, that $V$ is a separable strongly ordered Banach space and $X$ is a nonempty, open and order-convex subset of $V$. ( $X$ is called order-convex in $V$ iff $a, b \in X, x \in V$ and $a \leq x \leq b$ imply $x \in X$.) We also assume that the semiflow $\Phi: R_{+}^{1} \times X \rightarrow X$ is strongly increasing and $\omega$-compact in every simply ordered, compact arc $J \subset X$. Finally, we denote by $\hat{V}$ the set $V$ with the order-topology generated by all open order intervals $[[a, b]]=\{x \in V: a \ll x \ll b\}$ in $V$. The order-topology is obtained from any ordered norm on $V:$ Fix any $e \in \operatorname{Int}\left(V_{+}\right)$and define

$$
|x|_{e}=\inf \left\{\lambda \in \mathbf{R}_{+}^{1}:-\lambda e \leq x \leq \lambda e\right\} \text { for } x \in V \text {. }
$$

Theorem 0.1. Given any $x \in X$, precisely one of the following three alternatives occurs: 
(1) $x \in \mathcal{S}_{1 / 2}$ and $\omega(x)=\{p\} \subset \mathcal{E} \cap \mathcal{S}_{1 / 2}$ in which case $\Phi_{t} x \longrightarrow p$ as $t \longrightarrow \infty$.

(2) $x \in \mathcal{A}_{1 / 2}$ and $\omega(x) \subset \mathcal{E} \cap \mathcal{U}_{2}$ in which case there exist $p, q \in \mathcal{E}$ such that $\omega(x) \subset[[p, q]]$ and $\omega(y)=p$ and $\omega(z)=q$ whenever $p<y<w<z<q$ for some $w \in \omega(x)$. If, in addition, $V=\hat{V}$ and $\Phi$ admits a "strongly positive, compact linearization" about its equilibria (cf. Theorem 5.2), then $\omega(x)$ is a single $\omega$-biunstable equilibrium.

(3) $x \in \mathcal{U}_{2}$ and $\omega(x) \subset \mathcal{U}_{2}$ in which case there exist $p \ll q$ as in Alt. (2) above.

Finally, the sets $\mathcal{U}_{2} \subset \mathcal{U}=\mathcal{U}_{-} \cup \mathcal{U}_{+}$are "very small": Each $\mathcal{U}_{-}$and $\mathcal{U}_{+}$is the union of at most countably many Lipschitz manifolds of codimension one in $\hat{V}=\left(V,|\cdot|_{e}\right)$. In particular, $\mu(\mathcal{U})=0$ for every Gaussian measure $\mu$ on $V$.

This theorem is a combination of Theorems 5.1 and 5.2 with Propositions 4.4 and 5.3 and Corollary 4.5. A number of closely related results showing convergence to an equilibrium for the trajectory of every point in $X$ can be found in Alikakos \& Hess [2], Alikakos, Hess \& Matano [3], Aronsson \& Mellander [8], Henry [13], Hess [14], Hirsch [18-20, 23], Lajmanovich \& Yorke [27], Selgrade [36], Smith [39, 40], Smith \& Thieme [41, 42] and Takác [43-45]. Most of them assume at least one of the following three additional hypotheses: (i) $\mathcal{E} \subset \mathcal{S}$, cf. [43, 45], or even $X=\mathcal{S}$, cf. $[2,3,13,14,18]$; (ii) $\mathcal{E}$ contains no triplet $p<q<r$, cf. [41, 45], or even $\mathcal{E}$ is a singleton, cf. $[13,20,23]$; and (iii) $X \subset V$ is star-shaped from the origin and $\Phi_{t}$ is sublinear for each $t \in R_{+}^{1}$, cf. $[8,19,27,36,39,44]$. An interesting replacement of (i) (or $X=S$ ) was found by Hirsch [23, Sec. 2] who requires $V=R^{3}$ and $\Phi_{t}$ does not increase volume for each $t \in \mathbf{R}_{+}^{1}$ (rather than distance to achieve $X=\mathcal{S}$, cf. $[2,18]$ ). It is often not too difficult to verify an $\omega$-stability hypothesis imposed on an equilibrium or even a generic point $x \in X$. For instance, when dealing with an autonomous evolution equation which admits a linearization, one can apply the Principle of linearized stability, cf. Henry [13, Chap. 5].

A novelty in this article is an interesting description of the domain of attraction of the $\omega$-limit set $\omega(x)$ of a generic point $x \in \mathcal{S}_{1 / 2}$. Given $\emptyset \neq W \subset X$, the set $\mathcal{D}(W)=\{x \in X$ : $\omega(x) \subset W\}$ is called the domain of attraction of $W$. It follows from our Theorems 5.1 and 5.2 that, for any fixed $x \in S_{1 / 2}, \omega(x)$ can have only one of the following three forms, cf. Theorem 4.8, provided also $x \notin \partial_{-} \mathcal{U} \cap \partial_{+} \mathcal{U}$ :

Theorem 0.2. We have $\omega(x) \subset K$ where the set $K \subset X$ is defined by one of the following three alternatives:

(i) $K=\{p\}$, for some $p \in \mathcal{E} \cap \mathcal{A}_{1 / 2}$;

(ii) $K=P^{*}$, where $P^{*}$ is a maximal subset of $\mathcal{E}$ with the following property: $P^{*}$ is the image of a strictly increasing continuous path $P: I \longrightarrow X$ defined in a nondegenerate interval $I \subset \mathbf{R}^{1}$;

(iii) $K=\omega\left(x_{0}\right)$, for some $x_{0} \in \mathcal{A}_{1 / 2}$ such that $\omega\left(x_{0}\right) \subset \mathcal{E} \cap\left(\mathcal{U}_{2} \cup \partial_{-} \mathcal{U} \cup \partial_{+} \mathcal{U}\right)$.

The domain of attraction of $K$ from Theorem 0.2 has the following "shape": 
relatively compact for every $x \in J$, and also $\bigcup_{x \in J} \omega(x)$ is relatively compact in $X$. Given any $x \in X$, we first define the lower and upper $\omega$-limit sets of $x$, respectively, by

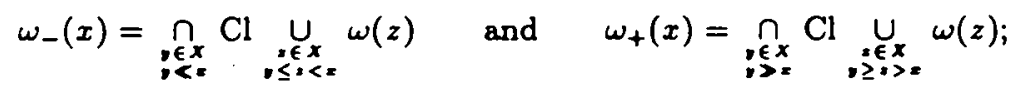

their properties are described in Proposition 3.4 through Corollary 3.7. Here "Cl" denotes the closure in $X$.

A point $x \in X$ is called lower $\omega$-stable (symbolically $x \in S_{-}$) if $\omega_{-}(x)=\omega(x)$; otherwise $x$ is lower $\omega$-unstable $\left(x \in \mathcal{U}_{-}\right)$. A point $x \in \mathcal{S}_{-}$is called lower asymptotically $\omega$-stable $\left(x \in \mathcal{A}_{-}\right)$if $\omega(y)=\omega(x)$ for some $y \in X, y<x$. The upper $\omega$-stability classification of $x \in X$ is defined analogously with $\omega_{+}(x)$ in place of $\omega_{-}(x)$. We set $\mathcal{S}=\mathcal{S}_{-} \cap \mathcal{S}_{+}$, $\mathcal{S}_{1 / 2}=\mathcal{S}_{-} \cup \mathcal{S}_{+}, \mathcal{U}=\mathcal{U}_{-} \cup \mathcal{U}_{+}, \mathcal{U}_{2}=\mathcal{U}_{-} \cap \mathcal{U}_{+}, \mathcal{A}=\mathcal{A}_{-} \cap \mathcal{A}_{+}$and $\mathcal{A}_{1 / 2}=\mathcal{A}_{-} \cup \mathcal{A}_{+}$.

Our most important result describing the asymptotic behavior of $\Phi$, Theorem 5.1 supplemented by Proposition 4.4 and Corollary 4.5, shows that "almost all" points $x \in X$ are order $\omega$-stable, i.e., $x \in \mathcal{S}$, and either $\omega(x) \subset \mathcal{S}_{1 / 2}$ is a single equilibrium, or else $\omega(x) \subset \mathcal{U}_{2}$ is a set of equilibria with $x \in \mathcal{A}_{1 / 2}$. We denote by $\mathcal{E}=\left\{x \in X: \Phi_{t} x=x\right.$ for all $\left.t \in \mathbf{R}_{+}^{1}\right\}$ the set of all equilibria. The main part of this result, namely $\omega(x) \subset \mathcal{E}$ for "almost all" $x \in X$, was proved already by Hirsch [20, Thm. 7.5 and 7.8] and later in a more general space setting by Smith \& Thieme [41, Thm. 3.3]. For a very common class of semiflows associated with autonomous evolution equations, e.g., irreducible cooperative systems of ordinary differential equations or semilinear parabolic partial differential equations, it was shown by Polácik [32, Thm. 1] and Smith \& Thieme [42, Prop. 2.5] that $\omega(x)$ is a single equilibrium for every $x \in \mathcal{S}$, cf. also our Theorem 5.2. Our approach has an interesting addition to their results, namely, description of $\mathcal{U}_{-}$and $\mathcal{U}_{+}$as the unions of at most countably many Lipschitz manifolds, thus showing that Smale's example [37] is, in fact, rather general. Our study of $\omega$-unstable points was inspired by Hirsch [21, Thm. 1.1 and Prop. 2.6]. Furthermore, $\mathcal{U}_{-} \subset \partial_{+} \mathcal{A}$ and $\mathcal{U}_{+} \subset \partial_{-} \mathcal{A}$, where $\partial_{-}$and $\partial_{+}$denote the lower and upper boundaries, resp., cf. Section 4. In particular, a strongly increasing continuous-time dynamical system $(X, \Phi)$ cannot be "very chaotic"; all "chaos" must occur inside $\mathcal{U}_{2}$, the set of all $\omega$-biunstable points. To state this and other results precisely we will assume, for simplicity, that $V$ is a separable strongly ordered Banach space and $X$ is a nonempty, open and order-convex subset of $V$. ( $X$ is called order-convex in $V$ iff $a, b \in X, x \in V$ and $a \leq x \leq b$ imply $x \in X$.) We also assume that the semiflow $\Phi: R_{+}^{1} \times X \longrightarrow X$ is strongly increasing and $\omega$-compact in every simply ordered, compact arc $J \subset X$. Finally, we denote by $\hat{V}$ the set $V$ with the order-topology generated by all open order intervals $[[a, b]]=\{x \in V: a \ll x \ll b\}$ in $V$. The order-topology is obtained from any ordered norm on $V$ : Fix any $e \in \operatorname{Int}\left(V_{+}\right)$and define

$$
|x|_{e}=\inf \left\{\lambda \in \mathbf{R}_{+}^{1}:-\lambda e \leq x \leq \lambda e\right\} \text { for } x \in V .
$$

Theorem 0.1. Given any $x \in X$, precisely one of the following three alternatives occurs: 
This is the Continuity principle in Proposition 3.10. Our proofs of Propositions 0.5 and 0.6 and many other results employ Hirsch's Limit set dichotomy [20, Thm. 0.8]:

Proposition 0.7. Let $a, b \in X, a<b$. Then either (a) $\omega(a) \ll \omega(b)$, or else (b) $\omega(a)=\omega(b)$.

This article is organized as follows. In Section 1 we prove the existence and smoothness of invariant $d$-hypersurfaces. In Section 2 we prove the existence of an invariant order resolution of $X$. Most of the results from Sec. 1 and 2 were already published in Takác [43, 45]. In Section 3 we study the continuity properties of the set-valued mapping $\omega: X \longrightarrow X$. In Section 4 we investigate the domain of attraction of a generic $\omega$-limit set, cf. Theorem 4.8. Finally, in Section 5 we prove several deeper results about the asymptotic behavior of continuous-time semiflows. Our main contribution here is a new description of $\mathcal{U}_{2}$, the set of all $\omega$-biunstable points. Analogous results for discrete-time semigroups $\left\{T^{n}: n \in Z_{+}\right\}$ are proved in Takáć [45].

Numerous applications of our results to autonomous evolution equations are well-known; e.g., irreducible cooperative systems of ordinary differential equations, semilinear parabolic partial differential equations, and some functional differential equations with delay. The reader is referred to Henry [13], Hirsch [19, Chap. III, Sec. 6] and [20, §3-5], Smith [40] and Smith \& Thieme [42]. Therefore we present only two examples, 2.4 and 2.5. Many examples from population and cell biology, ecology and epidemiology can be found in Fife [11], Friedman [12], Hethcote \& Yorke [15], Othmer [29] and Selgrade [36].

\section{Invariant $d$-hypersurfaces}

In Takác $[43,45]$ the author studied the existence and some smoothness properties of the so-called $d$-hypersurfaces (cf. Definition 1.1 below) which are invariant under a given discretetime semigroup $\left\{T^{n}: n \in Z_{+}\right\}$of nonlinear mappings acting on a strongly ordered space $X$. This study was inspired by a result of Hirsch [21, Prop. 2.6] for $X \subset \mathbf{R}^{N}$, the $N$-dimensional Euclidean space. In this section we will obtain the existence of $d$-hypersurfaces which are invariant under a given continuous-time semifiow $\Phi \equiv\left\{\Phi_{t}: t \in R_{+}^{1}\right\}$ acting on $X$. We start with some notation and a few definitions. We set $\mathbf{Z}_{+}=\{0,1,2, \cdots\}$ and $\boldsymbol{R}_{+}^{1}=[0, \infty)$. Throughout the entire paper we assume the following four hypotheses $(X),(V),(T)$ and $(\Phi)$ :

$(X): X$ is an ordered, metrizable topological space, i.e., $X$ is a metrizable topological space with a closed (partial) order relation " $\leq$ " in $X \times X$ (shortly, $X$ is an ordered space). We write $x \ll y$ if $(x, y)$ belongs to the interior of the order relation in $X \times X$, while $x<y$ means $x \leq y, x \neq y$. 
Theorem 0.3. (i) Both $\mathcal{D}(K)$ and $\ln t(\mathcal{D}(K))$ are order-convex and nonempty.

(ii) There exist two invariant (under $\Phi$ ) Lipschitz hypersurfaces $H_{1}$ and $H_{2}$ in $\dot{V}$ with $H_{1}$ below $H_{2}$ (i.e., $x \in H_{1}$ and $y \in H_{2}$ never satisfy $x>y$ ), both unordered and such that $\partial_{-} \mathcal{D}(K) \subset H_{1}, \partial_{+} \mathcal{D}(K) \subset H_{2}$ and $\operatorname{Fr}(\mathcal{D}(K)) \subset H_{1} \cap H_{2}$ (where "Fr" denotes the frame in Section 4).

(iii) The hypersurfaces $H_{1}$ and $H_{2}$ from (ii) can be chosen such that also $\operatorname{Int}(\mathcal{D}(K))$ is the union of some components of the set $W \subset X \backslash\left(H_{1} \cup H_{2}\right)$ squeezed between $H_{1}$ and $H_{2}$ (i.e., for all $x, y \in X$, we have $y \notin W$ whenever either $y \leq x \in H_{1}$ or $y \geq x \in H_{2}$, whereas $\|[x, y]] \subset W$ whenever $x \in H_{1}$ and $\left.y \in H_{2}\right)$. If $K \cap S_{1 / 2} \neq 0$ then $\operatorname{Int}(\mathcal{D}(K))$ is also connected.

This theorem is a consequence of our Theorem 4.8. The Lipschitz hypersurfaces occurring here are the so-called d-hypersurfaces studied in Prop. 1.3. In general, we define them to be the boundary $H=A \cap B$ of an order decomposition $(A, B)$ of $X$, cf. Definition 1.1, which is called invariant if $\Phi_{t}(A) \subset A$ and $\Phi_{t}(B) \subset B$, for all $t \in R_{+}^{1}$. The existence of invariant $d$-hypersurfaces is rather abundant, cf. Prop. 1.2: Every nonempty, unordered, invariant set $G \subset X$ is contained in some invariant d-hypersurface $H \subset X$.

Even a far more powerful result is valid:

Theorem 0.4. Let $\Lambda$ be any (possibly empty) simply ordered system of invariant order decompositions of $X$, i.e., $\left(A_{1}, B_{1}\right),\left(A_{2}, B_{2}\right) \in \Lambda \Longrightarrow A_{1} \subset A_{2}$ or $A_{2} \subset A_{1}$. Then $\Lambda$ is contained in another such system $\Gamma$ satisfying also the following maximality condition: If $x \in X$ has unordered orbit, then $\mathcal{O}^{+}(x) \subset H=A \cap B$ for some $(A, B) \subset \Gamma$.

This is our Theorem 2.2; the system $\Gamma$ is called an invariant order resolution of $X$. We refer to Hirsch [21, Thm. 1.1] for a related result stated for a competitive system of autonomous ordinary differential equations; a time reversal yields a cooperative system. This theorem and the following two propositions are, in fact, our key tools:

Proposition 0.5. Let $x \in U_{-}$, i.e., $\omega_{-}(x) \neq \omega(x)$. Then $\omega_{-}(x)$ is a single equilibrium in $\mathcal{A}_{+}$, and there exists $a \in X, a \ll x$, such that $\omega(y)=\omega_{-}(x)$ for all $y \in X, a \leq y<x$. Analogously for $x \in \mathcal{U}_{+}$.

This is the Discontinuity principle stated as Proposition 3.6.

Proposition 0.6. Let $J \subset X$ be a simply ordered, compact arc with endpoints $a<b$ satisfying $\omega(a) \neq \omega(b)$. Assume $a \in \mathcal{S}_{+}, b \in \mathcal{S}_{-}$and $J^{0}=J \backslash\{a, b\} \subset \mathcal{S}$. Then $\omega(x)$ is a single equilibrium in $\mathcal{S}_{1 / 2}$ for every $x \in J$, and $\bigcup_{x \in J} \omega(x)=F$ for some simply ordered, compact arc $F \subset \mathcal{E}$. 
This is the Continuity principle in Proposition 3.10. Our proofs of Propositions 0.5 and 0.6 and many other results employ Hirsch's Limit set dichotomy [20, Thm. 0.8]:

Proposition 0.7. Let $a, b \in X, a<b$. Then either (a) $\omega(a) \ll \omega(b)$, or else (b) $\omega(a)=\omega(b)$.

This article is organized as follows. In Section 1 we prove the existence and smoothness of invariant $d$-hypersurfaces. In Section 2 we prove the existence of an invariant order resolution of $X$. Most of the results from Sec. 1 and 2 were already published in Takáć [43, 45]. In Section 3 we study the continuity properties of the set-valued mapping $\omega: X \longrightarrow X$. In Section 4 we investigate the domain of attraction of a generic $\omega$-limit set, cf. Theorem 4.8. Finally, in Section 5 we prove several deeper results about the asymptotic behavior of continuous-time semiflows. Our main contribution here is a new description of $\mathcal{U}_{2}$, the set of all $\omega$-biunstable points. Analogous results for discrete-time semigroups $\left\{T^{n}: n \in Z_{+}\right\}$ are proved in Takáć [45].

Numerous applications of our results to autonomous evolution equations are well-known; e.g., irreducible cooperative systems of ordinary differential equations, semilinear parabolic partial differential equations, and some functional differential equations with delay. The reader is referred to Henry [13], Hirsch [19, Chap. III, Sec. 6] and [20, §3-5], Smith [40] and Smith \& Thieme [42]. Therefore we present only two examples, 2.4 and 2.5. Many examples from population and cell biology, ecology and epidemiology can be found in Fife [11], Friedman [12], Hethcote \& Yorke [15], Othmer [29] and Selgrade [36].

\section{Invariant $d$-hypersurfaces}

In Takác $[43,45]$ the author studied the existence and some smoothness properties of the so-called $d$-hypersurfaces (cf. Definition 1.1 below) which are invariant under a given discretetime semigroup $\left\{T^{n}: n \in Z_{+}\right\}$of nonlinear mappings acting on a strongly ordered space $X$. This study was inspired by a result of Hirsch [21, Prop. 2.6] for $X \subset \mathbf{R}^{N}$, the $N$-dimensional Euclidean space. In this section we will obtain the existence of $d$-hypersurfaces which are invariant under a given continuous-time semiflow $\Phi \equiv\left\{\Phi_{\ell}: t \in R_{+}^{1}\right\}$ acting on $X$. We start with some notation and a few definitions. We set $Z_{+}=\{0,1,2, \cdots\}$ and $\mathbf{R}_{+}^{1}=[0, \infty)$. Throughout the entire paper we assume the following four hypotheses $(X),(V),(T)$ and $(\Phi)$ :

$(X): X$ is an ordered, metrizable topological space, i.e., $X$ is a metrizable topological space with a closed (partial) order relation " $\leq$ " in $X \times X$ (shortly, $X$ is an ordered space). We write $x \ll y$ if $(x, y)$ belongs to the interior of the order relation in $X \times X$, while $x<y$ means $x \leq y, x \neq y$. 
$(V): \quad V$ is a strongly ordered, metrizable topological vector space (shortly, strongly ordered vector space), which is equivalent to saying that the positive cone $V_{+}=\{x \in V$ : $x \geq 0$ ) of $V$ has nonempty interior denoted by $\operatorname{lnt}\left(V_{+}\right)$. (In some of our results we will assume that $X$ is a nonempty subset of $V$ with closure $\mathrm{Cl}(X)$.)

$(T): T$ is a continuous, strongly increasing mapping of $X$ into itself, i.e., $x, y \in X$ and $x<y$ implies $T x \ll T y$.

$(\Phi): \Phi$ is a strongly increasing semiflow in $X$, i.e., a mapping $\Phi: \mathbf{R}_{+}^{1} \times X \longrightarrow X$ which satisfies:

Continuity: $\Phi$ is continuous in $R_{+}^{1} \times X$.

Determinism: $\Phi(0, x)=x$ and $\Phi(s+t, x)=\Phi(t, \Phi(s, x))$ for all $x \in X$ and $s, t \in R_{+}^{1}$.

Strong monotonicity: $\Phi(t, \cdot): X \longrightarrow X$ is strongly increasing for every $t>0$.

(In most of our results we will assume that $T=\Phi_{r} \equiv \Phi(\tau, \cdot)$ for some fixed $\tau>0$.) We write $\Phi_{t} \equiv \Phi(t, \cdot): X \longrightarrow X$ for $t \in R_{+}^{1}$, and identify $\Phi \equiv\left\{\Phi_{t}: t \in R_{+}^{1}\right\}$.

An ordered space $X$ is called strongly ordered if every open subset $U$ of $X$ satisfies:

(SO1) If $x \in U$ then $a \ll x \ll b$ for some $a, b \in U$.

It is easy to see that, for every open subset $U$ of $X,(S O 1)$ implies:

(SO2) If $a, b \in U$ and $a \ll b$ then $a \ll x \ll b$ for some $x \in U$.

E.g., every nonempty, open subset of $V$ is a strongly ordered space.

The positive semiorbit (shortly, orbit) of any $x \in X$ is defined by $\mathcal{O}^{+}(x)=\left\{\Phi_{\imath} x: t \in\right.$ $\left.\mathbf{R}_{+}^{1}\right\}$, and the $\omega$-limit set of $x$ is defined by $\omega(x)=\left\{y \in X: \Phi_{l_{n}} x \longrightarrow y(n \longrightarrow \infty)\right.$ for some sequence $t_{n} \longrightarrow \infty$ in $R_{+}^{1}$ \}. A subset $Y$ of $X$ is called positively invariant (shortly, invariant) if $\Phi_{t}(Y) \subset Y$ for each $t \in \mathbf{R}_{+}^{1}$, and totally invariant if $\Phi_{t}(Y)=Y$ for each $t \in \mathbf{R}_{+}^{1}$. For instance, every $\mathcal{O}^{+}(x)$ is invariant, and every $\omega(x)$ with $\mathcal{O}^{+}(x)$ relatively compact is totally invariant. Moreover, $\mathcal{O}^{+}(x)$ is connected, and if it is also relatively compact in $X$ then also $\omega(x)$ is connected, compact and nonempty.

Analogous concepts are defined for the discrete-time semigroup $\left\{T^{n}: n \in Z_{+}\right\}$: The orbit of $x \in X$ is defined by $\mathcal{O}_{T}^{+}(x)=\left\{T^{n} x: n \in Z_{+}\right\}$, and the $\omega$-limit set of $x$ is defined by $\omega_{T}(x)=\left\{y \in X: T^{n} x \longrightarrow y(k \longrightarrow \infty)\right.$ for some sequence $n_{k} \longrightarrow \infty$ in $\left.Z_{+}\right\}$. Notice that if $\mathcal{O}_{T}^{+}(x)$ is relatively compact in $X$, then $\omega_{T}(x) \neq 0$. A subset $Y$ of $X$ is called $T$-invariant if $T(Y) \subset Y$, and totally $T$-invariant if $T(Y)=Y$. For instance, every $\mathcal{O}_{T}^{+}(x)$ is $T$-invariant, and every $\omega_{T}(x)$ with $\mathcal{O}_{T}^{+}(x)$ relatively compact is totally $T$-invariant.

If $T=\Phi_{r}$ for a fixed $\tau>0$, the continuous-time and discrete-time orbits of $x \in X$ are obviously related by

$$
\mathcal{O}^{+}(x)=\cup_{0 \leq \imath<T} \Phi_{\imath}\left(\mathcal{O}_{T}^{+}(x)\right)
$$

If, in addition, $K_{T}=\mathrm{Cl}\left(\mathcal{O}_{T}^{+}(x)\right)$ is compact in $X$, then so is $K=\mathrm{Cl}\left(\mathcal{O}^{+}(x)\right)$, by $K \subset$ $\Phi\left([0, \tau] \times K_{T}\right)$, and we have also

$$
\omega(x)=\cup_{0 \leq t<r} \Phi_{1}\left(\omega_{T}(x)\right)
$$


Given $a, b \in X$, the set $[a, b]=\{x \in X: a \leq x \leq b\}$ is called a closed order interval, and $[[a, b]\}=\{x \in X: a \ll x \ll b\}$ is called an open order interval in $X$. We write $[a, \infty]]=\{x \in X: x \geq a\}$, and similarly for $[[-\infty, b]$ etc. A subset $Y$ of $X$ is called: order-convex in $X$ if $[a, b] \subset Y$ whenever $a, b \in Y$ and $a<b$; lower closed if $[[-\infty, b] \subset Y$ whenever $b \in Y$; and upper closed if $[a, \infty]] \subset Y$ whenever $a \in Y$.

We denote closed order intervals in $V$ by $[a, b]_{V}=\{x \in V: a \leq x \leq b\}$, and similarly, all other concepts in $V$ will be marked by the subscript $V$ in case confusion might arise.

Now we are ready to introduce our crucial concept:

Definition 1.1. A pair $(A, B)$ of subsets $A, B$ of $X$ is called an order decomposition of $X$ if it has the following five properties:

(i) $A \neq \emptyset$ and $B \neq \emptyset$;

(ii) $A$ and $B$ are closed;

(iii) $A$ is lower closed and $B$ is upper closed,

(iv) $A \cup B=X$; and

(v) $\operatorname{Int}(A \cap B)=0$.

An order decomposition $(A, B)$ of $X$ is called invariant if $\Phi_{1}(A) \subset A$ and $\Phi_{1}(B) \subset B$, for each $t \in \mathbf{R}_{+}^{1}$. Analogously, $(A, B)$ is called $T$-invariant if $T(A) \subset A$ and $T(B) \subset B$.

The set $H=A \cap B$ (possibly empty) is called the boundary of the order decomposition $(A, B)$ of $X$. A d-hypersurface is any nonempty subset $H$ of $X$ such that $H=A \cap B$ for some order decomposition $(A, B)$ of $X$.

Notice that the boundary $H$ of an order decomposition $(A, B)$ of $X$ satisfies $H=\partial A=$ $\partial B$, where " $\partial$ " is the boundary symbol in $X$, and $H$ is invariant whenever $(A, B)$ is invariant. It is also easy to see that a $d$-hypersurface $H$ never contains two strongly ordered points $x$, $y$ (with $x \ll y$ ). Consequently, if $H$ is invariant then it must be unordered, i.e., no pair of points $x, y \in H$ satisfies $x<y$.

If $X$ is a strongly ordered space it turns out to be very useful to work with the order topology on $X$ whose neighborhood base is generated by all open order intervals $[[a, b]]$ with $a \ll b$. If $Y \subset X$, we denote by $\hat{Y}$ the set $Y$ endowed with the induced order topology. A subset $Y$ of $X$ is called order-open (order-closed, resp.) if it is open (closed, resp.) in $\dot{X}$. Notice that the identity mapping $\hat{i}: X \longrightarrow \hat{X}$ is continuous, but in general not homeomorphic. It is proved in Hirsch [20] that if $f: X_{1} \longrightarrow X_{2}$ is a continuous, increasing mapping between two strongly ordered spaces (i.e., $x \leq x_{1} y$ implies $f(x) \leq x_{2} f(y)$ ), then $f$ is continuous also in the order topologies, that is, the induced map $\hat{f}: \bar{X}_{1} \longrightarrow \hat{X}_{2}$ is continuous. It is easy to see that the order topology on $V$ is induced by any ordered norm $1 \cdot l_{e}$ on $V$ defined by

$$
|x|_{e}=\inf \left\{\lambda \in \mathbf{R}_{+}^{1}:-\lambda e \leq x \leq \lambda e\right\}
$$

for some $e \in \operatorname{Int}\left(V_{+}\right)$. 
Our first result guarantees the existence of invariant $d$-hypersurfaces, and our second result describes them as Lipschitz hypersurfaces:

Proposition 1.2. Let $X$ and $\Phi$ satisfy $(X)$ and $(\Phi)$, and let $X$ be strongly ordered. Assume that $G$ is a nonempty, unordered, invariant subset of $X$. Then there exists an invariant order decomposition $(A, B)$ of $X$ such that $G \subset H=A \cap B$. In particular, we can define $(A, B)$ in either of the following two ways:

(a) $A=\operatorname{Cl}\left(A^{\circ}\right)$ and $B=X \backslash A^{\circ}$ where $A^{\circ} \stackrel{\text { def }}{=}\left\{x \in X: \Phi_{1} x \ll y\right.$ for some $t \in \mathbf{R}_{+}^{1}$ and $y \in G\}$;

(b) $A=X \backslash B^{\circ}$ and $B=\mathrm{Cl}\left(B^{\circ}\right)$ where $B^{\circ} \stackrel{\text { def }}{=}\left\{x \in X: \Phi_{t} x \gg y\right.$ for some $t \in \mathbf{R}_{+}^{1}$ and $y \in G\}$.

For instance, we may take $G=\omega(x)$ for any relatively compact $\mathcal{O}^{+}(x)$, cf. Proposition 3.2 below.

We recall that an everywhere defined linear mapping $L: V_{1} \rightarrow V_{2}$ between two ordered vector spaces is called positive (strongly positive, resp.) if $x<y$ in $V_{1}$ implies $L x \leq L y(L x \ll L y$, resp. $)$ in $V_{2}$. We set $I=$ identity mapping on $V$, and $\mathbf{R}^{1}=(-\infty, \infty)$.

Proposition 1.3. Let $X$ be a nonempty open subset of $V$, and let $(A, B)$ be an order decomposition of $X$ with the boundary $H=A \cap B$. Fix any vector $v \in \ln \left(V_{+}\right)$, and denote by $R=\operatorname{lin}\{v\}$ the linear subspace of $V$ spanned by $v$. Let $Q$ be a positive continuous projection of $V$ onto $R$, which always exists, and set $P=I-Q$ with $W=P(V)$, the range of $P$, so that $V=W \oplus R$ is the direct algebraic and topological sum of $W$ and $R$. Then we have the following statements:

(i) The restriction $\left.P\right|_{H}$ of $P$ to $H$ is one-to-one, and both $\left.P\right|_{H}$ and its inverse $\pi=$ $\left(\left.P\right|_{H}\right)^{-1}: P(H) \longrightarrow H$ are Lipschitz continuous in the ordered norm $|\cdot|_{0}$ with a common Lipschitz constant 2.

(ii) $\left.P\right|_{H}$ is a homeomorphism of $H$ onto $P(H)$ in the topologies induced by that on $V$.

(iii) Furthermore, set

$$
H \oplus R=\left\{x \in V: x=x_{0}+\tau v \quad \text { for some } x_{0} \in H \text { and } \tau \in R^{1}\right\}
$$

where $x_{0}$ and $\tau$ are uniquely determined by $P_{x}=P_{x_{0}}$, and define a mapping $h: H \oplus R \longrightarrow$ $\checkmark$ by

$$
h(x)=P x_{0}+\tau v, \quad x=x_{0}+\tau v \in H \oplus R,
$$

and similarly for $P(H) \oplus R$. Then also $h$ and its inverse $h^{-1}: P(H) \oplus R \longrightarrow H \oplus R$ are Lipschitz continuous in the ordered norm $|\cdot|_{v}$ with a common Lipschitz constant 7 , and $h$ is a homeomorphism of $H \oplus R$ onto $P(H) \oplus R$ in the topologies induced by that on $V$. 
(iv) If, in addition, $X$ is order-open in $V$ (i.e., open in $\hat{V}$ ), then $P(H)$ is order-open in $W$, and $P(H) \oplus R$ is order-open in $V$.

Only Part (i) of Proposition 1.3 will be needed later on. Notice that Proposition 1.2 supplemented by Proposition 1.3 bears resemblance to a version of the Hahn-Banach theorem, cf. Schaefer [35, Sec. V.5.4, Cor. 2], which guarantees the existence of the projection $Q$ in Proposition 1.3. The first version of Part (i) was proved by Hirsch [21, Prop. 2.6] for the case $\operatorname{dim}(V)<\infty$, the dimension of $V$. We refer to Takáć [43, Prop. 1.2] or [45, Prop. 1.3] for a proof of Proposition 1.3. Our proof of Proposition 1.2 is based on the following two lemmas from Takác $[43,45]$ :

Lemma 1.4. Let $X$ be a strongly ordered space. If $F \subset X$ is lower closed (upper closed, resp.), then so are its interior $\operatorname{Int}(F)$ and closure $\mathrm{Cl}(F)$, while its complement $X \backslash F$ is upper closed (lower closed, resp.). The union and intersection of any family of lower (upper) closed sets are lower (upper) closed.

ProOF. Let $F \subset X$. It is clear that $F$ is lower closed if and only if $X \backslash F$ is upper closed. Now assume that $F$ is lower closed. Consider any $x \in \operatorname{Int}(F)$. Then also $y \in \operatorname{Int}(F)$ for some $y \gg x$ because $X$ is strongly ordered. Consequently, we have $[[-\infty, y] \subset F$ since $F$ is lower closed. Hence, $[[-\infty, x] \subset \operatorname{Int}[[-\infty, y] \subset \operatorname{Int}(F)$ shows that $\operatorname{Int}(F)$ is lower closed. Analogously, $\operatorname{Int}(X \backslash F)$ is upper closed which in turn implies that $\operatorname{Cl}(F)=X \backslash \operatorname{Int}(X \backslash F)$ is lower closed. The remaining claims are trivial.

Lemma 1.5. Let $X$ be a strangly ardered space. Assume that $\left(A^{\prime}, B^{\prime}\right)$ is a pair of subsets of $X$ satisfying properties (i), (iii), (iv) and (v) of Definition 1.1. Set $A=\mathrm{Cl}\left(A^{\prime}\right)$ and $B=\mathrm{Cl}\left(B^{\prime}\right)$. Then $(A, B)$ is an order decomposition of $X$. Furthermore, if both $A^{\prime}$ and $B^{\prime}$ are $T$-invariant, then so is $(A, B)$, for any mapping $T$ satisfying $(T)$.

Proof. Properties (i), (ii) and (iv) are obvious while (iii) follows from Lemma 1.4. It remains to show property $(\mathrm{v})$, i.e., $\operatorname{Int}(A \cap B)=0$. Suppose $b \in \operatorname{Int}(A \cap B) \neq 0$. Then also $z \ll b \ll w$ for some $z$, $w \in \operatorname{Int}(A \cap B)$, since $X$ is strongly ordered, and consequently $z^{\prime} \ll b \ll w^{\prime}$ for some $z^{\prime} \in B^{\prime}$ and $w^{\prime} \in A^{\prime}$. Hence $b \in\left[\left[z^{\prime}, w^{\prime}\right]\right] \subset A^{\prime} \cap B^{\prime}$ since $A^{\prime}$ is lower closed and $B^{\prime}$ is upper closed. But then $b \in \operatorname{Int}\left(A^{\prime} \cap B^{\prime}\right)$ contradicts $(v)$ for $\left(A^{\prime}, B^{\prime}\right)$.

PrOOF of Proposition 1.2. We prove only (a), the proof of (b) being analogous. So let $(A, B)$ be defined by (a). Clearly, $A^{\circ}$ is open since each $\Phi_{l}\left(t \in \mathbf{R}_{+}^{1}\right)$ is continuous, and $A^{\circ}$ is lower closed since each $\Phi_{\mathrm{l}}$ is increasing. Lemma 1.4 shows that $A=\operatorname{Cl}\left(A^{\circ}\right)$ is lower closed, whereas $B=X \backslash A^{\circ}$ is upper closed. Hence, the pair $\left(A^{\circ}, B\right)$ satisfies properties (i), 
(iii), (iv) and (v) in Definition 1.1. We apply Lemma 1.5 to conclude that $(A, B)$ is an order. decomposition of $X$.

Furthermore, making use of the fact that each $\Phi_{t}\left(t \in \mathbf{R}_{+}^{1}\right)$ preserves the strong ordering "《", we arrive at $\Phi_{\imath}\left(A^{\circ}\right) \subset A^{\circ}$ and $\Phi_{\imath}(B) \subset B$. Hence, $(A, B)$ is invariant by Lemma 1.5 .

Finally, since $G$ is invariant and contains no pair of strongly ordered points $x \ll y$, we have $A^{\circ} \cap G=0$. On the other hand, $X$ is strongly ordered implies $G \subset \mathrm{Cl}(\underset{y \in G}{\cup}[[-\infty, y]\})$, and in particular $G \subset \mathrm{Cl}\left(A^{\circ}\right)$. Consequently $G \subset \mathrm{Cl}\left(A^{\circ}\right) \backslash A^{\circ}=\partial A^{\circ}=A \cap B$ as desired. This finishes our proof of Prop. 1.2.

\section{Invariant order resolutions}

In this section we will obtain a considerably more powerful result than Prop. 1.2, namely, the existence of an invariant order resolution of $X$, cf. Theorem 2.2. This result will turn out to be very useful for our investigation of the domain of attraction of a generic $\omega$-limit set (in Section 4).

Definition 2.1. A system $\Gamma$ of invariant order decompositions of $X$ (cf. Definition 1.1) is called an invariant order resolution of $X$ if it satisfies the following two conditions:

Simple ordering: If $\left(A_{1}, B_{1}\right),\left(A_{2}, B_{2}\right) \in \Gamma$ then either $A_{1} \subset A_{2}$ or $A_{2} \subset A_{1}$.

Maximality: If $x \in X$ and $\mathcal{O}^{+}(x)$ is unordered, then $x \in H=A \cap B$ for some $(A, B) \in \Gamma$.

Notice that $x \in X$ and $\mathcal{O}^{+}(x)$ is relatively compact implies $\omega(x) \subset H=A \cap B$ for some $(A, B) \in \Gamma$, by Proposition 3.2. In particular, if $x \notin H$ for every $(A, B) \in \Gamma$, then $\omega(x)$ is a single equilibrium $p \in X$, i.e., $\Phi_{t} p=p, t \in \mathbf{R}_{+}^{1}$.

The main result in this section is the following analogue of a theorem due to Hirsch [2], Thm. 1.1] for irreducible competitive systems of autonomous ordinary differential equations:

Theorem 2.2. (Invariant order resolution.) Let $X$ and $\Phi$ satisfy (X) and ( $\Phi)$, and let $X$ be strongly ordered. Assume that $\Lambda$ is a (possibly empty) system of invariant order decompositions of $X$ satisfying the simple ordering condition from Definition 2.1. Then there exists an invariant order resolution $\Gamma$ of $X$ such that $\Lambda \subset \Gamma$.

We introduce the following ordering " $\subseteq$ " of invariant order decompositions of $X$ : We write $\left(A_{1}, B_{1}\right) \subseteq\left(A_{2}, B_{2}\right)$ if and only if $A_{1} \subset A_{2}\left(\Longleftrightarrow B_{2} \subset B_{1}\right)$. Observe that, if $X$ is an open order interval in a strongly ordered topological vector space $V$, then we have: $\left(A_{1}, B_{1}\right) \subseteq\left(A_{2}, B_{2}\right)$ if and only if $H_{1} \preceq H_{2}$, where $H_{i}=A_{i} \cap B_{i}$ for $i=1,2$. Here $H_{1} \preceq H_{2}$ 
means the following: If $x \in H_{1}$ then $x \leq y$ for some $y \in H_{2}$, and if $y \in H_{2}$ then $x \leq y$ for some $x \in H_{1}$.

Our proof of Theorem 2.2 hinges on the following technical lemma from Takác [45].

Lemma 2.3. Let $X$ and $\Phi$ satisfy $(X)$ and $(\Phi)$, and let $X$ be strongly ordered. Let $\left(A_{1}, B_{1}\right)$ and $\left(A_{2}, B_{2}\right)$ be invariant order decompositions of $X$ with $\left(A_{1}, B_{1}\right) \subseteq\left(A_{2}, B_{2}\right)$. Assume that $G$ is a nonempty, unordered, invariant subset of $Y=\operatorname{Int}\left(A_{2}\right) \cap \operatorname{Int}\left(B_{1}\right)$. Then there exists a third invariant order decomposition $(A, B)$ of $X$ such that $G \subset H=A \cap B$ and

$$
\left(A_{1}, B_{1}\right) \subseteq(A, B) \subseteq\left(A_{2}, B_{2}\right) .
$$

A similar statement holds if $A_{1}=0$ or $B_{2}=0$.

Proof. Since $X$ is strongly ordered, $A_{2}$ lower closed and $B_{1}$ upper closed, we conclude that $Y$ is an open, order convex subset of $X$. In particular, $Y$ is a strongly ordered space. Observe that $Y$ is invariant, because both $\operatorname{Int}\left(A_{2}\right)$ and $\operatorname{Int}\left(B_{1}\right)$ are invariant by the strong monotonicity of each $\Phi_{t}, t \in R_{+}^{1} \backslash\{0\}$. By Proposition 1.2 there exists an invariant order decomposition $\left(A_{3}, B_{3}\right)$ of $Y$ such that $G \subset H_{3}=A_{3} \cap B_{3}$. We define $A^{\prime}=A_{1} \cup A_{3}$ and $B^{\prime}=B_{2} \cup B_{3}$ and also $A=\mathrm{Cl}\left(A^{\prime}\right)$ and $B=\mathrm{Cl}\left(B^{\prime}\right)$ in $X$. Clearly $A_{1} \subset A \subset A_{2}$ and $B_{2} \subset B \subset B_{1}$. We claim that $(A, B)$ is an invariant order decomposition of $X$.

First we have:

(i) $A^{\prime} \neq 0$ and $B^{\prime} \neq 0$. (Trivial.)

(ii) $A$ and $B$ are closed. (Trivial.)

(iii) $A^{\prime}$ is lower closed and $B^{\prime}$ is upper closed. Proof: Take any $x \in A^{\prime}$. If $x \in A_{1}$ then [ $[-\infty, x] \subset A_{1} \subset A^{\prime}$. If $x \in A_{3} \backslash A_{1}$ then $\left[[-\infty, x] \subset A_{1} \cup\left([[-\infty, x] \cap Y) \subset A_{1} \cup A_{3}\right.\right.$ since $Y$ is order-convex in $X$ and $A_{3}$ is lower closed in $Y$. Thus, $A^{\prime}$ is lower closed, and analogously $B^{\prime}$ is upper closed.

(iv) $A^{\prime} \cup B^{\prime}=X$. Proof: We have $A^{\prime} \cup B^{\prime}=\left(A_{1} \cup B_{2}\right) \cup\left(A_{3} \cup B_{3}\right)=(X \backslash Y) \cup Y=X$.

(v) $\operatorname{Int}\left(A^{\prime} \cap B^{\prime}\right)=0$. Proof: Set $H_{i}=A_{i} \cap B_{i}$ for $i=1,2,3$. Then

$$
A^{\prime} \cap B^{\prime}=\left(A_{1} \cup A_{3}\right) \cap\left(B_{2} \cup B_{3}\right)=\left(A_{1} \cap B_{2}\right) \cup\left(A_{3} \cap B_{3}\right) \subset\left(H_{1} \cap H_{2}\right) \cup H_{3}
$$

since $A_{1} \cup B_{2}=X \backslash Y$ and $A_{3} \cup B_{3}=Y$. Now suppose $\operatorname{Int}\left(A^{\prime} \cap B^{\prime}\right) \neq 0$. Since $H_{1} \cap H_{2}$ is closed with $\operatorname{Int}\left(H_{1} \cap H_{2}\right)=\emptyset$, we conclude that $Z=\operatorname{Int}\left(A^{\prime} \cap B^{\prime}\right) \backslash\left(H_{1} \cap H_{2}\right)$ is open and nonempty. But also $Z \subset H_{3}$ which contradicts $\operatorname{Int}\left(H_{3}\right)=\emptyset$. So (v) is valid.

It follows from Lemma 1.5 that $(A, B)$ is an order decomposition of $X$ which is invariant because both $A^{\prime}$ and $B^{\prime}$ are invariant as unions of invariant sets. This concludes the proof.

P ROOF of Theorem 2.2. Let $\mathcal{G}$ denote the collection of all systems $\Gamma$ of invariant order decompositions of $X$ satisfying the simple ordering condition form Definition 2.1 and 
$\Lambda \subset \Gamma$. We order $\mathcal{G}$ by inclusion " $\subset$ ". Clearly $\mathcal{G} \neq 0$ by $\Lambda \in \mathcal{G}$, even if $\Lambda=\emptyset$. Furthermore, every simply ordered nonempty subset $\mathcal{G}^{\prime}$ of $\mathcal{G}$ has an upper bound in $\mathcal{G}$, namely, the system $\Gamma^{\prime}=U\left\{\Gamma: \Gamma \in \mathcal{G}^{\prime}\right\}$. Hence, we may apply Zorn's lemma to conclude that $\mathcal{G}$ possesses a maximal element, say, $\Gamma_{0}$. We claim that $\Gamma_{0}$ satisfies also the maximality condition from Definition 2.1.

On the contrary, suppose there exists $x \in X$ such that $\mathcal{O}^{+}(x)$ is unordered and $x \notin$ $H=A \cap B$ for every $(A, B) \in \Gamma_{0}$. Hence, either $x \in \operatorname{Int}(A)$ or else $x \in \operatorname{Int}(B)$. We define the subsystems.

$$
\Gamma_{0}^{1}=\left\{(A, B) \in \Gamma_{0}: x \in \operatorname{Int}(B)\right\} \quad \text { and } \quad \Gamma_{0}^{2}=\left\{(A, B) \in \Gamma_{0}: x \in \operatorname{Int}(A)\right\} .
$$

Observe that $F_{0}^{1} \cup \Gamma_{0}^{2}=\Gamma_{0}$ and $\Gamma_{0}^{1} \cap \Gamma_{0}^{2}=0$. Next we set

$$
A_{1}^{\prime}=\cup\left\{A:(A, B) \in \Gamma_{0}^{1}\right\} \quad \text { and } \quad B_{1}^{\prime}=\cap\left\{\operatorname{lnt}(B):(A, B) \in \Gamma_{0}^{1}\right\}
$$

and analogously

$$
A_{2}^{\prime}=\cap\left\{\operatorname{lnt}(A):(A, B) \in \Gamma_{0}^{2}\right\} \quad \text { and } \quad B_{2}^{\prime}=\cup\left\{B:(A, B) \in \Gamma_{0}^{2}\right\} .
$$

It is easy to see that both $A_{1}^{\prime}$ and $A_{2}^{\prime}$ are lower closed, while both $B_{1}^{\prime}$ and $B_{2}^{\prime}$ are upper closed, by Lemma 1.4. Furthermore, $A_{i}^{\prime} \cup B_{i}^{\prime}=X$ and $A_{i}^{\prime} \cap B_{i}^{\prime}=\emptyset$ for $i=1,2$, and $A \subset A_{1}^{\prime} \subset A_{2}^{\prime}$ for each $(A, B) \in \Gamma_{0}^{1}$, while $A_{1}^{\prime} \subset A_{2}^{\prime} \subset A$ for each $(A, B) \in \Gamma_{0}^{2}$. Finally, all $A_{1}^{\prime}, A_{2}^{\prime}, B_{1}^{\prime}$ and $B_{2}^{\prime}$ are invariant, and $x \in A_{2}^{\prime} \cap B_{1}^{\prime}$.

From now on we assume that $A_{1}^{\prime} \neq 0$ and $B_{2}^{\prime} \neq \emptyset$; the other cases are similar. We set $A_{i}=\mathrm{Cl}\left(A_{i}^{\prime}\right)$ and $B_{i}=\mathrm{Cl}\left(B_{i}^{\prime}\right)$ for $i=1,2$. Applying Lemma 1.5 we obtain that $\left(A_{i}, B_{i}\right)$ is an invariant order decomposition of $X$. Moreover, we have $A \subset A_{1} \subset A_{2}$ for each $(A, B) \in \Gamma_{0}^{1}, A_{1} \subset A_{2} \subset A$ for each $(A, B) \in \Gamma_{0}^{2}$, and $x \in A_{2} \cap B_{1}$. The maximality of $\Gamma_{0}$ in $\mathcal{G}$ implies $\left(A_{i}, B_{i}\right) \in \Gamma_{0}$, and in particular $\left(A_{i}, B_{i}\right) \in \Gamma_{0}^{i}$ for $i=1,2$. Hence $x \in Y=\operatorname{Int}\left(A_{2}\right) \cap \operatorname{Int}\left(B_{1}\right)$, where $Y$ is invariant by the strong monotonicity of each $\Phi_{\ell}, t \in \mathbf{R}_{+}^{1} \backslash\{0\}$. We employ Lemma 2.3 to obtain an invariant order decomposition $(\tilde{A}, \tilde{B})$ of $X$ such that $G=\mathcal{O}^{+}(x) \subset \tilde{H}=\tilde{A} \cap \bar{B}$ and $\left(A_{1}, B_{1}\right) \subseteq(\tilde{A}, \tilde{B}) \subseteq\left(A_{2}, B_{2}\right)$. Thus $(\tilde{A}, \tilde{B}) \notin \Gamma_{0}$, but the system $\Gamma_{1}=\Gamma_{0} \cup\{(\tilde{A}, \tilde{B})\}$ is still simply ordered; a contradiction with the maximality of $\dot{\Gamma}_{0}$ in $\mathcal{G}$. We conclude that $\Gamma_{0}$ is an invariant order resolution of $X$, and $\Lambda \subset \Gamma_{0}$.

The following is a simple example of an invariant order resolution for a semilinear time-independent parabolic partial differential equation in divergence form:

Example 2.4. Consider the initial-boundary value problem

$$
\begin{array}{lll}
(I V P): & \frac{\partial u}{\partial t}=\sum_{i=1}^{N} \frac{\partial}{\partial x_{i}}\left[\sum_{j=1}^{N} a_{i j}(x) \frac{\partial u}{\partial x_{j}}+a_{i}(x, u)\right] & \text { in } \Omega \times(0, \infty) ; \\
& \sum_{i=1}^{N} n_{i}(x)\left[\sum_{j=1}^{N} a_{i j}(x) \frac{\partial u}{\partial x}+a_{i}(x, u)\right]=0 & \text { on } \partial \Omega \times(0, \infty) ; \\
u(x, 0)=u_{0}(x) \quad \text { in } \Omega .
\end{array}
$$


Here $\Omega \subset \mathbf{R}^{N}$ is an open bounded domain of class $C^{\infty},\left[a_{i j}(x)\right]_{i, j=1}^{N}$ is a uniformly po definite matrix for $x \in \Omega$ and satisfies $a_{i j} \in C^{\infty}(\bar{\Omega})$, and $a_{i} \in C^{\infty}\left(\bar{\Omega} \times \mathbf{R}^{1}\right)$ with

$$
\frac{\partial a_{i}}{\partial u}, \frac{\partial^{2} a_{i}}{\partial x_{i} \partial u} \text { and } \frac{\partial^{2} a_{i}}{\partial u^{2}}
$$

uniformly bounded in $\Omega \times \mathbf{R}^{\mathbf{1}}$. Finally $\left[n_{i}(x)\right]_{i=1}^{N}$ denotes the outer unit normal to $i$ $x \in \partial \Omega$. We assume that all $a_{i j}$ and $a_{i}$ are real-valued. To guarantee existence, uniqu and continuous dependence on the initial data of a mild solution $u$ to (IVP) by simple tr cf. Amann [5, Thm. 15.1, Cor. 15.3], Ladyzhenskaya, Solonnikov \& Uraltseva [26, ( V., Thm. 7.4] or Pazy $[30$, Sec. 8.4], we assume that our boundary conditions are line

$$
a_{i}(x, u)=\hat{a}_{i}(x) u \quad \text { on } \partial \Omega \times R^{1} .
$$

The reader is referred to Amann [7, Thm. 6.1, Cor. 6.2] for more general boundary c tions.

We denote by $C^{\alpha}(\bar{\Omega})(0 \leq \alpha<3)$ the Hölder space and by $W_{p}^{\sigma}(\Omega)(N<p$. $\left.1+\frac{N}{p}<\sigma<2\right)$ the Sobolev-Slobodeckii space, see e.g., Adams [1] or Triebel [46]. the imbedding $W_{p}^{\sigma}(\Omega) \hookrightarrow C^{1}(\bar{\Omega})$ is continuous, all these spaces are strongly ordered B: spaces. We set either $V=C^{\alpha}(\bar{\Omega})$ or $V=W_{p}^{o}(\Omega)$. Using Pazy's results [30] we obt unique mild (semigroup) solution $u: \mathbf{R}_{+}^{1} \longrightarrow L^{2}(\Omega)$ to (IVP) which depends continuou: the initial data $u_{0} \in L^{2}(\Omega)$. We define a mapping $\tilde{\Phi}: \mathbf{R}_{+}^{1} \times L^{2}(\Omega) \longrightarrow L^{2}(\Omega)$ by $\tilde{\Phi}_{t} u_{0}=$ $t \in \mathbf{R}_{+}^{1}$, which is continuous. Applying the regularity theory of Ladyzhenskaya et al. I $V=C^{\alpha}(\bar{\Omega})$, and Amann [5] if $V=W_{p}^{\sigma}(\Omega)$, we conclude that $\tilde{\Phi}_{t}(V) \subset V$ and the restri $\Phi_{t}=\left.\tilde{\Phi}_{t}\right|_{V}: V \longrightarrow V$ of $\tilde{\Phi}_{t}$ to $V$ is continuous and order-compact for every $t>0$. Mort if $u_{0} \in V$ then $u \in C^{2,1}(\bar{\Omega} \times(0, \infty))$ and $u$ is a classical solution of (IVP). Hence $\Phi_{t}$, i is strongly increasing by the strong maximum and boundary point principles, cf. Prot Weinberger [34, Chap. 3, Sec. 3], and the integral

$$
\int_{\Omega} u(x, t) d x, \quad t \in \mathbf{R}_{+}^{1}
$$

is independent from $t \geq 0$, by the divergence theorem. In other words, the set

$$
H_{\rho}=\left\{f \in V: \int_{\Omega} f(x) d x=\rho\right\}, \quad \text { for every } \rho \in \mathbf{R}^{1},
$$

is an invariant $d$-hypersurface in $V$; we have $H_{\rho}=A_{\rho} \cap B_{\rho}$ where the sets

$$
A_{\rho}=\left\{f \in V: \int_{\Omega} f(x) d x \leq \rho\right\} \quad \text { and } \quad B_{\rho}=\left\{f \in V: \int_{\Omega} f(x) d x \geq \rho\right\}
$$

form an invariant order decomposition of $V$. We conclude that the system $\Gamma=\left\{\left(A_{\rho}\right.\right.$ $\rho \in \mathbf{R}^{\mathbf{1}\}}$ is an invariant order resolution of $V$. This system reflects the conservation c 
integral in (1) and satisfies $H_{\rho} \nless H_{\sigma}$ whenever $-\infty<\rho<\sigma<\infty$. Here $H_{\rho} \nless H_{0}$ means the following: If $x \in H_{\rho}$ then $x \ll y$ for some $y \in H_{\sigma}$, and if $y \in H_{\sigma}$ then $x \ll y$ for some $x \in H_{\rho}$.

Another example of an invariant order resolution is the following one:

Example 2.5. Consider the irreducible cooperative system of autonomous ordinary differential equations with reaction-type nonlinearities:

$$
\frac{d}{d t}\left[\begin{array}{l}
x_{1} \\
x_{2}
\end{array}\right]=\left[\begin{array}{rr}
-1 & 1 \\
1 & -1
\end{array}\right]\left[\begin{array}{l}
x_{1} \\
x_{2}
\end{array}\right]+\left[\begin{array}{l}
f\left(x_{1}\right) \\
f\left(x_{2}\right)
\end{array}\right]
$$

where $f(x)=-\alpha x(x-1)^{2}, x \in R^{1}$, with a constant $\alpha>0$. Given any initial values $\left(x_{1}(0), x_{2}(0)\right)=\left(x_{10}, x_{20}\right) \in \mathbf{R}^{2}$, this system has a unique solution $\left(x_{1}(t), x_{2}(t)\right) \equiv$ $\phi_{t}\left(x_{10}, x_{20}\right)$ which exists locally in time for $0 \leq t<t_{0}$ where $t_{0}=t_{0}\left(x_{10}, x_{20}\right), 0<t_{0} \leq \infty$, denotes the escape time of $\left(x_{10}, x_{20}\right)$. The mappings $\phi_{t}: \mathbf{R}^{2} \longrightarrow \mathbf{R}^{2}, t \in \mathbf{R}_{+}^{1}$, form a local semi-flow $\phi$ in $\mathbf{R}^{2}$, and each $\phi_{t}, t>0$, is strongly increasing in its domain in $\mathbf{R}^{2}$, by Kamke's theorem, see e.g., Hirsch [19, Chap. III., Sec. 1]. That $\phi$ is complete, i.e., $t_{0}=\infty$ for all $\left(x_{10}, x_{20}\right) \in \mathbf{R}^{2}$, follows from the strong monotonicity of each $\phi_{t}, t>0$, and the following identity, for $t \in \mathbf{R}_{+}^{\mathbf{1}}$ :

$$
\frac{d}{d t}\left(x_{1}+x_{2}\right)=-\alpha\left[x_{1}\left(x_{1}-1\right)^{2}+x_{2}\left(x_{2}-1\right)^{2}\right]
$$

where $\left(x_{1}(t), x_{2}(t)\right)=\phi_{t}\left(x_{10}, x_{20}\right)$. implies:

Namely, observe that $p=(0,0)$ and $q=(1,1)$ are equilibria of $\phi$, and our identity

(i) If $\left(x_{10}, x_{20}\right) \leq p$ or $p \leq\left(x_{10}, x_{20}\right)<q$, then $\phi_{t}\left(x_{10}, x_{20}\right) \longrightarrow p$ as $t \rightarrow \infty$, since both $\left[[-\infty, p]\right.$ and $[p, q] \backslash\{q\}$ are invariant under each $\phi_{l}$, and $\frac{d}{d i}\left(x_{1}+x_{2}\right)>0$ (< 0 , resp.) for $\left(x_{1}, x_{2}\right)<p\left(p<\left(x_{1}, x_{2}\right)<q\right)$.

(ii) If $\left(x_{10}, x_{20}\right) \geq q$ then $\phi_{8}\left(x_{10}, x_{20}\right) \longrightarrow q$ as $t \rightarrow \infty$, since $\left.[q, \infty]\right]$ is invariant under each $\phi_{t}$, and $\frac{d}{d t}\left(x_{1}+x_{2}\right)<0$ for $\left(x_{1}, x_{2}\right)>q$.

Our final goal in this example is to show that every point $\left(x_{10}, x_{20}\right) \in \mathbf{R}^{2}$ is attracted by either $p$ or else $q$, and to determine the domain of attraction of each $p$ and $q$, i.e., the set

$$
\mathcal{D}(p)=\left\{\left(x_{10}, x_{20}\right) \in \mathbf{R}^{2}: \phi_{t}\left(x_{10}, x_{20}\right) \longrightarrow p \text { as } t \longrightarrow \infty\right\}
$$

and similarly for $q$. With regard to Theorem 2.2 (and Prop. 3.1 below), it suffices to construct an invariant order resolution $\Gamma$ for the dynamical system $(V, \Phi)$, where $V=\mathbf{R}^{2}$ and $\Phi=\phi$, such that $\Gamma$ consists of precisely two invariant order decompositions $\left(A_{p}, B_{p}\right)$ and $\left(A_{q}, B_{q}\right)$ with $p \in H_{p}=A_{p} \cap B_{p}$ and $q \in H_{q}=A_{q} \cap B_{q}$. Obviously, it follows from (i) 
and (ii) combined with the strong monotonicity of $\phi$, that every $\left(x_{10}, x_{20}\right) \in \mathbf{R}^{2}$ is attracted by the union

$$
\bar{Y}=[1-\infty, p] \cup[p, q] \cup[q, \infty]],
$$

and consequently by either $p$ or else $q$. Furthermore, we have $\mathcal{E}=\{p, q\}$ with $p$ attracting $[[-\infty, q] \backslash\{q\}$ and $q$ attracting $\{q, \infty]]$. Hence, it remains to determine the invariant $d$ hypersurfaces $H_{p}$ and $H_{q}$ together with their uniqueness. Since our system of ordinary differential equations is symmetric with respect to $x_{1}$ and $x_{2}$, the uniqueness of $H_{p}$ and $H_{q}$ will imply that both $H_{p}$ and $H_{q}$ are symmetric with respect to the axis $x_{1}=x_{2}$. Thus, we set $V^{\prime}=\left\{\left(x_{1}, x_{2}\right) \in V: x_{1} \leq x_{2}\right\}$ and $V^{\prime \prime}=\left\{\left(x_{1}, x_{2}\right) \in V: x_{1} \geq x_{2}\right\}$, and compute only the unique curves $H_{p} \cap V^{\prime}$ and $H_{q} \cap V^{\prime}$ starting at $p$ and $q$, resp. To compute these curves we introduce the following new coordinate system in $V$ :

$$
\xi=\frac{1}{2}\left(x_{2}-x_{1}\right), \quad \eta=\frac{1}{2}\left(x_{1}+x_{2}\right) .
$$

It is easy to see that both these curves are graphs of a function $\eta=\eta(\xi)$ of $\xi \in \mathbf{R}_{+}^{1}$ which satisfies the following ordinary differential equation:

$$
\begin{aligned}
\frac{d \eta}{d \xi} & =\frac{(\xi+\eta)(\xi+\eta-1)^{2}-(\xi-\eta)(\xi-\eta+1)^{2}}{\frac{\frac{1}{\alpha} \xi+(\xi+\eta)(\xi+\eta-1)^{2}+(\xi-\eta)(\xi-\eta+1)^{2}}{}} \\
& =\frac{\frac{1}{\xi} \eta(\eta-1)^{2}+\xi(3 \eta-2)}{\frac{2}{\alpha}+\xi^{2}+(\eta-1)(3 \eta-1)}
\end{aligned}
$$

for $\xi>0$. This is the equation for the integral curves of our original system of ordinary differential equations. These curves are uniformly Lipschitz continuous if and only if at $\xi=0$ we have either $\eta=0$ or $\eta=1$. It is obvious that, for these values of $\xi$ and $\eta$, they are unique and have the Maclaurin series

$$
\eta(\xi)=a_{0}+a_{2} \xi^{2}+a_{4} \xi^{4}+\cdots, \quad 0 \leq \xi<\xi_{0}
$$

for some $\xi_{0}>0$, where $a_{0}=\eta(0)(=0$ or 1$), a_{2} \neq 0$, etc. In particular, both $H_{p}$ and $H_{q}$ are analytic curves diffeomorphic to $\mathbf{R}^{\mathbf{1}}$. Finally, we have $\mathcal{D}(p)=\operatorname{Int}\left(A_{q}\right)$ and $\mathcal{D}(q)=B_{q}$.

\section{Lower and upper $\omega$-limit sets}

Let $X$ and $\Phi: R_{+}^{1} \times X \longrightarrow X$ satisfy $(X)$ and $(\Phi)$. We denote by $\mathcal{E}=\left\{x \in X: \Phi_{t} x=x\right.$ for all $\left.t \in \mathbf{R}_{+}^{1}\right\}$ the set of all equilibria (i.e., stationary points) of $\Phi$. We start with the following elementary results which are due to Hirsch [20, Thm. 6.4 and 6.2]:

Proposition 3.1. (Convergence criterion for strongly monotone semiflows.) Let $X$ and $\Phi$ satisfy $(X)$ and $(\Phi)$. Assume that $x \in X, \mathcal{O}^{+}(x)$ is relatively compact, and either 
$\Phi_{r} x>x$ or $\Phi_{r} x<x$ for some $\tau>0$. Then $\Phi_{l} x \longrightarrow p$ as $t \rightarrow \infty$, for some $p \in \mathcal{E}$, and either $p \ll x$ or $p \gg x$, respectively.

Proposition 3.2. (Nonordering of limit sets.) Let $X$ and $\Phi$ satisfy $(X)$ and $(\Phi)$ Assume that $x \in X$ and $\mathcal{O}^{+}(x)$ is relatively compact. Then $\omega(x)$ is nonempty and unordered. If $\mathrm{Cl}\left(\mathcal{O}^{+}(x)\right)$ is not unordered, then $\omega(x)$ is a single equilibrium.

ProOf. This result is a direct consequence of Proposition 3.1, cf. Takáč [43, Lemma 2.2].

These two propositions are essential for the proof of Hirsch's Limit set dichotomy [20, Thm. 6.8] for strongly increasing semiflows.

Proposition 3.3. (Limit set dichotomy.) Let $X$ and $\Phi$ satisfy $(X)$ and $(\Phi)$. Let $x$, $y \in X, x<y$, and assume that $\mathcal{O}^{+}(x) \cup \mathcal{O}^{+}(y)$ is relatively compact in $X$. Then either

(a) $\omega(x) \ll \omega(y)$ (in which case $\omega(x) \cap \omega(y)=0$ ) or else

(b) $\omega(x)=\omega(y) \subset \mathcal{E}$ (in which case, for any sequence $t_{k} \in \mathbf{R}_{+}^{1}, t_{k} \longrightarrow \infty$, and for any $w \in \omega(x)=\omega(y)$ we have: $\left.\Phi_{t_{k}} x \longrightarrow w \Longleftrightarrow \Phi_{t_{k}} y \longrightarrow w\right)$.

Consequently, the $\omega$-limit sets of points with relatively compact orbits are partially ordered by " $\ll$ ". We write $A \leq B(A<B, A \ll B$, resp.) for $A, B \subset X$ whenever $x \leq y$ $(x<y, x \ll y)$ holds for all $x \in A$ and $y \in B$.

Throughout the remaining part of this section we assume that $X$ and $\Phi$ satisfy $(X)$ and $(\Phi)$, and $X$ is strongly ordered. We say that the semiflow $\Phi$ is $\omega$-compact in a subset $Y$ of $X$ if $\mathcal{O}^{+}(x)$ is relatively compact for each $x \in Y$, and also $\bigcup_{x \in Y} \omega(x)$ is relatively compact in $X$. For such $Y$ and $\Phi$ we define the lower and upper $\omega$-limit sets of $x \in Y$ relative to $Y$ by

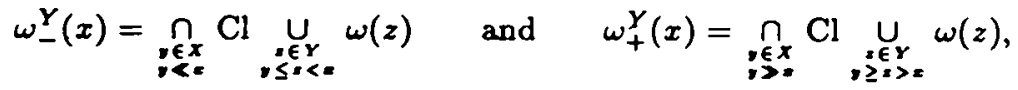

respectively. Observe that if there exists a sequence $z_{n} \in Y, z_{n}<x\left(z_{n}>x\right.$, resp.) and $z_{n} \longrightarrow x$, then $\omega_{-}^{Y}(x) \neq \emptyset\left(\omega_{+}^{Y}(x) \neq \emptyset\right)$, by the $\omega$-compactness of $\Phi$.

Under the $\omega$-compactness hypothesis for $\Phi$ in a suitable subset $Y$ of $X$ we will be able to describe some important properties of the set-valued mapping $\omega: X \longrightarrow X$. Wie start with the following analogue of Proposition 3.2:

Proposition 3.4. (Nonordering of lower and upper limit sets.) Let $X$ and $\Phi$ sativfy $(X)$ and $(\Phi)$, and let $X$ be strongly ordered. Assume that $x \in Y \subset X$ and $\Phi$ is $\omega$-corripart in $Y$. Then both $\omega_{-}^{Y}(x)$ and $\omega_{+}^{Y}(x)$ are compact, totally invariant and unordered. 
P ROOF. We consider only $\omega_{-}^{Y}(x)$. It is compact because $\Phi$ is $\omega$-compact. Since $\Phi_{\mathbf{t}}(\mathrm{Cl}(K))=\mathrm{Cl}\left(\Phi_{\mathbf{t}}(K)\right), t \in \mathbf{R}_{+}^{1}$, for any relatively compact set $K \subset X$, we have also $\Phi_{t} \omega_{-}^{Y}(x)=\omega_{-}^{Y}(x)$. To prove that $\omega_{-}^{Y}(x)$ is unordered, we suppose there exist $a, b \in \omega_{-}^{Y}(x)$ with $a<b$. But $\Phi_{1} a \ll \Phi_{1} b$ and $\omega_{-}^{Y}(x)$ is totally invariant imply that we may assume $a \ll b$. Hence, there exists $y \in Y, y<x$, such that $a \ll b^{*}$ for some $b^{*} \in \omega(y)$. Now choose $z \in Y, z<x$, such that $\Phi_{1} y \leq \Phi_{1} z \ll \Phi_{1} x$ and $a^{*} \ll b^{*}$ for some $a^{*} \in \omega(z)$. Then there exists a sequence $t_{k} \in \mathbf{R}_{+}^{1}, t_{k} \longrightarrow \infty$ as $k \longrightarrow \infty$, such that $\Phi_{t_{k}} y \longrightarrow b^{*}$ and $\Phi_{t_{k}} z \longrightarrow c$ for some $c \in \omega(z)$. It follows that $a^{*} \ll b^{*} \leq c$ which shows that $\omega(z)$ is not unordered, a contradiction to Proposition 3.2. Therefore $\omega_{-}^{Y}(x)$ must be unordered.

Corollary 3.5. Let all hypotheses of Proposition 3.4 be satisfied. If $z_{n} \longrightarrow x$ for some sequence $z_{n} \in Y, z_{n}<x$, then

$$
\omega_{-}^{Y}(x)=\bigcap_{k=1}^{\infty} \mathrm{Cl} \bigcup_{n=k}^{\infty} \omega\left(z_{n}\right)
$$

and $\omega(y) \leq \omega_{-}^{Y}(x) \leq \omega(x)$ for each $y \in X, y<x$ and $\mathcal{O}^{+}(y)$ relatively compact. $A$ corresponding result holds for $\omega_{+}^{Y}(x)$.

ProOF. Since $\Phi_{1}$ is strongly increasing and continuous, hence also order-continuous, the sequence $\left\{z_{n}\right\}$ contains a subsequence denoted again by $\left\{z_{n}\right\}$ such that $\Phi_{1} z_{1} \ll \Phi_{1} z_{2} \ll$ $\cdots \ll \Phi_{1} x$. Therefore we may assume $z_{1} \ll z_{2} \ll \cdots \ll x$. Set $Z=\left\{x, z_{1}, z_{2}, \cdots\right\}$. Then $Z \subset Y$ shows that $\neq \omega_{-}^{Z}(x) \subset \omega_{-}^{Y}(x)$. Now take any $a \in \omega_{-}^{Y}(x)$ and choose a sequence $a_{k} \longrightarrow a$ in $X$ as $k \longrightarrow \infty$ such that $a_{1} \ll a_{2} \ll \cdots \ll a$. Then there exists a sequence $y_{k} \in Y, y_{k}<x$, such that $a_{k} \leq v_{k}$ for some $v_{k} \in \omega\left(y_{k}\right)$. Next choose $n_{k} \in N, n_{k} \nearrow \infty$ as $k \longrightarrow \infty$, such that $\Phi_{1} y_{k} \leq \Phi_{1} z_{n_{k}} \ll \Phi_{1} x$. Hence, there is $w_{k} \in \omega\left(z_{n_{k}}\right)$ satisfying $w_{k} \geq v_{k} \geq a_{k}$ for each $k \in N$. Since $\Phi$ is $\omega$-compact in $Y$, we can pass to a subsequence $w_{k_{m}} \longrightarrow w$ in $X$ as $m \longrightarrow \infty$. It follows that $w \in \omega_{-}^{Z}(x)$ and $w \geq a$. But $a, w \in \omega_{-}^{Y}(x)$ and $\omega_{-}^{Y}(x)$ is unordered force $a=w$. So we have proved our first claim

$$
\omega_{-}^{Y}(x)=\omega_{-}^{Z}(x)=\bigcap_{k=1}^{\infty} \mathrm{Cl} \bigcup_{n=k}^{\infty} \omega\left(z_{n}\right)
$$

Next we observe that $\omega\left(z_{n}\right) \leq \omega(x)$ for all $n \in N$, by Prop. 3.3. Since $\Phi$ is $\omega$-compact in $Y$, we conclude that $\omega_{-}^{Y}(x) \leq \omega(x)$.

Finally, fix any $y \in X, y<x$ with $\mathcal{O}^{+}(y)$ relatively compact. Then there exists $n_{0} \in N$ such that $\Phi_{1} y \leq \Phi_{1} z_{n} \ll \Phi_{1} x$ for all $n \geq n_{0}$. Hence, to prove $\omega(y) \leq \omega_{-}^{Y}(x)$ we may assume $y \leq z_{n}$ for all $n \geq 1$. It follows that $\omega(y) \leq \omega\left(z_{n}\right)$ for all $n \in N$, and so $\omega(y) \leq \omega_{-}^{Y}(x)$.

Remark. It is clear from Corollary 3.5 that the set $\omega_{-}^{Y}(x)\left(\omega_{+}^{Y}(x)\right.$, resp.) is independent from the choice of $Y \subset X$ such that $x \in Y, z_{n} \longrightarrow x$ for some sequence $z_{n} \in Y, z_{n}\left\langle x\left(z_{n}\right\rangle\right.$ 
$x$ ), and $\Phi$ is $\omega$-compact in $Y$. Therefore, we say that a point $x \in X$ is lower (upper, resp.) approximable if there exists a sequence $x_{n} \in X, x_{n}<x\left(x_{n}>x\right)$, satisfying $x_{n} \longrightarrow x$ and $\Phi$ is $\omega$-compact in $Z=\left\{x, x_{1}, x_{2}, \cdots\right\}$. We define the lower (upper) $\omega$-limit set of such $x$ by $\omega_{-}(x)=\omega_{-}^{Z}(x)\left(\omega_{+}(x)=\omega_{+}^{Z}(x)\right)$.

Now we can introduce the following stability classification of a lower (upper, resp.) approximable point $x \in X$ :

We say that $x$ is lower (upper) $\omega$-stable if $\omega_{-}(x)=\omega(x)\left(\omega_{+}(x)=\omega(x)\right)$; otherwise $x$ is lower (upper) w-urstable. We say that $x$ is lower (upper) asymptotically $\omega$-stable if there exists $y \in X, y<x(y>x)$, with $\mathcal{O}^{+}(y)$ relatively compact and $\omega(y)=\omega(x)$. The set of all lower (upper) $\omega$-stable points $x \in X$ is denoted by $\mathcal{S}_{-}\left(\mathcal{S}_{+}\right)$, the set of all lower (upper) $\omega$ unstable points by $U_{-}\left(U_{+}\right)$, and the set of all lower (upper) asymptotically $\omega$-stable points by $\mathcal{A}_{-}\left(\mathcal{A}_{+}\right)$.

Observe that our atability notions are equivalent to the continuity properties of the set-valued mapping $\omega: X \longrightarrow X$. The structure of the $\omega$-limit sets near an $\omega$-unstable point $x \in X$ is very simple:

Proposition 3.6. (Discontinuity principle.) Let $X$ and $\Phi$ satisfy $(X)$ and $(\Phi)$, and let $X$ be strongly ondered. Assume that $x \in U_{-}$. Then there exists $a \in X, a \& x$, such that $\omega(y)=\omega_{-}(x)$ for every $y \in X$ with $a \leq y<x$ and $O^{+}(y)$ relatively compact. Moreover, if the set

$$
Y_{0}=\left\{y \in X: \begin{array}{ll}
\mathcal{O}^{+}(y) \text { is relatively compact and } p<y<q \\
\text { for some } p \in \omega_{-}(x) \text { and } q \in \omega(x)
\end{array}\right\}
$$

is nonempty, then $\omega_{-}(x)$ is a single equilibrium, and $\omega(y)=\omega_{-}(x)$ for every $y \in Y_{0}$. In particular $\omega_{-}(x) \ll \omega(x)$.

A corresponding result holds for $x \in \mathcal{U}_{+}$.

ProOF. The set $\omega_{-}(x)$ is nonempty, totally invariant and unordered by Proposition 3.4 . Thus, we may apply Proposition 1.2 to obtain an invariant order decomposition $(A, B)$ of $X$ such that $\omega_{-}(x) \subset B=A \cap B$. Suppose $x \in A$. Then also $\omega(x) \subset A$. On the other hand, the inequality $\omega_{-}(x) \leq \omega(x)$ implies $\omega(x) \subset B$ whence $\omega(x) \subset H$. Then $\omega_{-}(x) \leq \omega(x)$ in $H$ and $H$ unordered imply $\omega_{-}(x)=\omega(x)$, a contradiction to our hypotheses. So we must have $x \in \operatorname{Int}(B)$ which entails $x \gg a$ for some $a \in B$. Now fix any $y \in X, a \leq y<x$ and $\mathcal{O}^{+}(y)$ relatively compact. Then $y \in B, \omega(y) \subset B$ and $\omega(y) \leq \omega_{-}(x)$ by Corollary 3.5 , which shows that $\omega(y) \subset A$. Again $\omega(y) \leq \omega_{-}(x)$ in $H$ and $H$ unordered force $\omega(y)=\omega_{-}(x)$ as desired.

Now fix any $y \in Y_{0} \neq 0$. Then also $\omega_{-}(x) \leq \omega(y) \leq \omega(x)$ by Prop. 3.3 combined with the Absorption principle of Hirsch [20, Prop. 6.9]. As above there exists an invariant r,der decomposition $(A, B)$ of $X$ such that $\omega(y) \subset H=A \cap B$. Consequently $\omega_{-}(x) \subset A$ and $\omega(x) \subset B$. We claim that $x \in \operatorname{Int}(B)=X \backslash A$. 
Suppose $x \in A$. Hence $\omega(x) \subset A \cap B=H$, and therefore $\omega(y) \leq \omega(x)$ in $H$ which, in turn, forces $\omega(y)=\omega(x)$. Next choose $p \in \omega_{-}(x)$ and $q \in \omega(x)$ such that $p<y<q$, by $y \in Y_{0}$. Since $\Phi_{1} p \ll \Phi_{1} y \ll \Phi_{1} q$ and $\Phi_{1} y \in Y_{0}$ by the invariance of $\omega_{-}(x)$ and $\omega(x)$, we may assume $p \ll y \ll q$. So we can find $\tau \in R_{+}^{1} \backslash\{0\}$ satisfying $y \ll \Phi_{r} x$. Since $\Phi_{r}$ is continuous, we can find also $b \in[[-\infty, x]]$ with $y \leq \Phi_{r} b$. In particular, for every $z \in X$ with $b \leq z \leq x$ and $\mathcal{O}^{+}(z)$ relatively compact, we have $\omega(z)=\omega(y)=\omega(x)$, thus obtaining $\omega_{-}(x)=\omega(x)$ and contradicting our hypotheses. We have verified $x \notin A$ as claimed.

Since $x \in \operatorname{Int}(B)$, we have $a^{*} \ll x$ for some $a^{*} \in B$. It follows that $\omega_{-}(x)=\omega(z) \subset B$ for every $z \in X$ with $\mathcal{O}^{+}(z)$ relatively compact and $a \leq z<x, a^{*} \leq z$; fix such a point $z$. We obtain $\omega_{-}(x) \subset H$, and so $\omega_{-}(x) \leq \omega(y)$ in $H$ forces $\omega(y)=\omega_{-}(x)$. As above we may assume $p \ll y \ll q$ for some $p \in \omega_{-}(x)$ and $q \in \omega(x)$. Again we can find $\tau \in \mathbf{R}_{+}^{1} \backslash\{0\}$ satisfying $\Phi_{r} z \ll y$ which implies $\omega_{-}(x)=\omega(z) \leq \omega(y)$. Consequently $\omega(y)=\omega_{-}(x)$ and $y \gg p \in \omega(y)$. Hence $\mathrm{Cl}\left(\mathcal{O}^{+}(y)\right)$ is not unordered, and we conclude that $\omega(y)$ must be a single equilibrium, by Proposition 3.2. This proves that $\omega_{-}(x)$ is a single equilibrium and $\omega_{-}(x) \ll \omega(x)$.

Remark. In Proposition 3.6 above let us consider the set

$$
Y=\left\{y \in X: p<y<q \text { for some } p \in \omega_{-}(x) \text { and } q \in \omega(x)\right\}
$$

Then $X$ is strongly ordered and $\omega_{-}(x) \ll \omega(x)$ imply $\operatorname{Int}(Y) \neq 0$. Thus, if every $y \in Y$ has relatively compact orbit $\mathcal{O}^{+}(y)$, we have $Y=Y_{0}$ and $\omega_{-}(x)$ is a single equilibrium attracting a nonempty open set.

A set $J \subset X$ is called a closed (or compact) are (open arc, resp.) in $X$ if it is homeomorphic to the closed (open) interval $[0,1] \subset R^{1}\left((0,1) \subset R^{1}\right)$. A set $Y \subset X$ is called simply (or totally) ordered if $x, y \in Y \Longrightarrow x \leq y$ or $x \geq y$. The following result is an easy consequence of Proposition 3.6 and the Remark thereafter.

Corollary 3.7. Let $X$ and $\Phi$ satisfy $(X)$ and $(\Phi)$, and let $X$ be strangly ordered. Assume that the set

$$
X_{0}=\left\{x \in X: \mathcal{O}^{+}(x) \text { is relatively compact }\right\}
$$

is dense in $X$. Let $J \subset X_{0}$ be a simply ordered, open arc. Then the set $J_{-}=J \cap U_{-}$is at most countable (i.e., either finite or countable), and for each $x \in J_{-}$there exists $a \in[[-\infty, x]]$ satisfying $[a, x] \cap J_{-}=\{x\}$ and $\omega(y)=\omega_{-}(x)=\{p\} \subset \mathcal{E}$ for all $y \in X_{0}, a \leq y<x$.

A corresponding result holds for $\mathcal{U}_{+}$. 
Remark. If in Corollary 3.7 the semiflow $\Phi$ is $\omega$-compact in $J$, then all but at most countably many points $x \in J$ are order $\omega$-stable, i.e., $x \in \mathcal{S}=\mathcal{S}_{-} \cap \mathcal{S}_{+}$. More precisely, we have

$$
J=(J \cap \mathcal{S}) \cup(J \cap \mathcal{U}), \quad \mathcal{S} \cap \mathcal{U}=0, \quad \text { and } J \cap \mathcal{U} \subset \partial_{J}(\operatorname{Int} J(J \cap \mathcal{A})),
$$

where $\mathcal{U}=\mathcal{U}_{-} \cup \mathcal{U}_{+}$is the set of all order $\omega$-unstable points and $\mathcal{A}=\mathcal{A}_{-} \cap \mathcal{A}_{+}$is the set of all asymptotically order $\omega$-stable points. The symbols $\partial_{J}$ and Int $_{J}$ refer to the relative topology on $J$.

The structure of $\omega$-limit sets near a simply ordered, closed arc $J \subset X$ with endpoints $a, b(a<b)$, whose points are $\omega$-stable and $\omega(a) \cap \omega(b)=0$, is not much more complicated than near an $\omega$-unstable point. We say that the restriction $\left.\omega\right|_{J}$ of $\omega: X \longrightarrow X$ to $J$ is continuous if $\Phi$ is $\omega$-compact in $J$ and $\omega_{-}(x)=\omega(x)$ for every $x \in J \backslash\{a\}$ and $\omega_{+}(x)=\omega(x)$ for every $x \in J \backslash\{b\}$. We start with the following technical lemma.

Lemma 3.8. Let $X$ and $\Phi$ satisfy $(X)$ and $(\Phi)$, and let $X$ be strongly ordered. Let $J$ be a simply ordered, closed arc in $X$. Assume that $\Phi$ is w-compact in $J$ and $\left.\omega\right|_{J}$ is continuous. Then every strictly increasing unbounded sequence in $\mathbf{R}_{+}^{1}$ contains a subsequence $t_{1}<t_{2}<\cdots$ such that $t_{k} \longrightarrow \infty$ and for every $x \in J$ we have

$$
\Phi_{1} x \longrightarrow \varphi \text { in } X \text { as } k \longrightarrow \infty, \text { for some } \varphi=\varphi(x) \in X
$$

Furthermore, $\varphi: J \longrightarrow X$ is increasing (i.e., $x_{1} \leq x_{2}$ implies $\varphi\left(x_{1}\right) \leq \varphi\left(x_{2}\right)$ ) and continuous. In particular, the image of $\varphi$ is either a simply ordered, closed arc in $X$ or else a singleton.

P ROOF. Denote by $a, b \in J$ the end-points of the arc $J$ satisfying $a<b$. Let $M$ be any countable dense subset of $J$. Applying a diagonal process we conclude that every strictly increasing unbounded sequence in $R_{+}^{1}$ contains a subsequence $t_{1}<t_{2}<\cdots$ such that $t_{k} \longrightarrow \infty$ and for every $x \in M$ we have

$$
\Phi_{t_{\Delta}} x \longrightarrow \varphi \text { in } X \text { as } k \longrightarrow \infty, \text { for some } \varphi=\varphi(x) \in \omega(x) .
$$

Since $\Phi_{t}, t \in \mathbf{R}_{+}^{1}$, is increasing, so is $\varphi: M \longrightarrow X$. Furthermore, the $\omega$-compactness of $\Phi$ shows that $\varphi(M)$ is relatively compact in $X$. Consequently, we can define

$$
\begin{gathered}
\varphi_{-}(x)=\sup \{\varphi(y): y \in M, y<x\} \text { for every } x \in J \backslash\{a\}, \quad \text { and } \\
\varphi_{+}(x)=\inf \{\varphi(y): y \in M, y>x\} \text { for every } x \in J \backslash\{b\} .
\end{gathered}
$$

It is easy to see that $\varphi_{-}(x) \in \omega_{-}(x)$ and $\varphi_{+}(x) \in \omega_{+}(x)$, respectively. 
Now consider any $x \in J \backslash\{a\}$; the case $x \in J \backslash\{b\}$ is analogous. Let $w \in \omega(x)$ be any limit point of the sequence $\Phi_{\imath}, x, k \in N$. Since $\Phi_{\imath}, t \in \mathbf{R}_{+}^{1}$, is increasing, we obtain $\varphi(y) \leq w$ for every $y \in M$ with $y<x$, and therefore $\varphi_{-}(x) \leq w$. By the continuity of $\omega \mid$, we have $\omega_{-}(x)=\omega(x)$, an unordered set. Thus $w=\varphi_{-}(x)$ and $\Phi_{t_{k}} x \longrightarrow \varphi_{-}(x)$ as $k \longrightarrow \infty$. We conclude that (1) bolds also for every $x \in J \backslash\{a\}$ where $\varphi=\varphi_{-}(x) \in \omega(x)$, and for every $x \in J \backslash\{b\}$ where $\varphi=\varphi_{+}(x) \in \omega(x)$. Hence, $\varphi$ can be extended to a continuous mapping from $J$ into $X$ by setting

$$
\varphi(a)=\varphi_{+}(a), \quad \varphi(b)=\varphi_{-}(b) \text { and } \varphi(x)=\varphi_{-}(x)=\varphi_{+}(x) \text { for } x \in J \backslash\{a, b\} .
$$

Then (1) holds for every $x \in J$, and $\varphi: J \longrightarrow X$ is increasing.

Finally, assume that $\varphi(J)$ is not a singleton. Then $\varphi(J)$ is a simply ordered continuum which is not a point. It follows that $\varphi(J)$ must be a simply ordered, closed arc in $X$, cf. Wilder [47, Chap. I, Thm. 11.12].

Following the conclusion of Lemma 3.8 we introduce the following concept:

Definition 3.8. Let $J$ be a simply ordered, closed arc in $X$ such that $\mathcal{O}^{+}(x)$ is relatively compact for every $x \in J$. A simply ordered, closed arc $F$ in $X$ is called an $\omega$-limit fibre of $J$ if there exist a sequence $t_{1}<t_{2}<\cdots$ in $\mathbf{R}_{+}^{1}$ and an increasing continuous mapping $\varphi: J \longrightarrow F$ of $J$ onto $F$ such that $t_{k} \longrightarrow \infty$ and $\Phi_{t_{k}} x \longrightarrow \varphi(x)$ in $X$ as $k \longrightarrow \infty$, for every $x \in J$.

The set of all $\omega$-limit fibres of a simply ordered, closed arc is either empty or a singleton:

Proposition 3.10. (Continuity principle.) Let $X$ and $\Phi$ satisfy $(X)$ and $(\Phi)$, and let $X$ be strongly ordered. Let $J \subset X$ be a simply ordered, closed arc with endpoints $a, b$ ( $a<b$ ) satisfying $\omega(a) \neq \omega(b)$. Assume that $\Phi$ is $\omega$-compact in $J$ and $\left.\omega\right|_{J}$ is continuous. Then $\omega(x)$ is a single equilibrium for every $x \in J$ and $\underset{x \in J}{\bigcup} \omega(x)=F$ is the only w-limit fibre of $J$.

ProOF. Since $\omega(a) \neq \omega(b)$, Prop. 3.3 gives us also $\omega(a) \ll \omega(b)$. Fix any $p \in \omega(a)$. By Lemma 3.8 , there exists an $\omega$-limit fibre $F$ of $J$ such that $p \in F$. So $p=\min (F)$. Since both $J$ and $F$ are simply ordered, compact arcs, we may apply Prop. 3.3 to conclude that $\omega(a) \ll z$ for all $z \in F \backslash\{p\}$. But this means $\omega(a) \leq p \in \omega(a)$ in an unordered set $\omega(a)$ which, in turn, forces $\omega(a)=\{p\} \subset \mathcal{E}$. Analogously $\omega(b)=\{q\} \subset \mathcal{E}$. Applying these two results to any $x \in J$ we arrive at $\omega(x)=\{r\} \subset \mathcal{E}$ and $\underset{x \in J}{\bigcup} \omega(x)=F \subset \mathcal{E}$ as desired.

Remark. In Proposition 3.10 we have $J^{0}=J \backslash\{a, b\} \subset \mathcal{S}$ by the continuity of $\left.\omega\right|_{J}$. Moreover, the set $J^{0} \backslash\left(\mathcal{A}_{-} \cup \mathcal{A}_{+}\right)$is infinite and uncountable. To see this we examine the 
napping $\varphi: J \longrightarrow F$ from Lemma 3.8. Take any $x \in J^{0}$ and observe that $x \in \mathcal{A}_{-} \cup \mathcal{A}_{+}$if und only if $J_{x}=\varphi^{-1}(\varphi(x))$ is a closed arc in $J$, because $\varphi$ is increasing and continuous. The eparability of $J$ implies that there are at most countably many $w \in F$ such that $\varphi^{-1}(w)$ is closed arc; otherwise $\varphi^{-1}(w)$ is a singleton contained in $J \backslash \mathcal{A}^{\prime}$ where $\mathcal{A}^{\prime}=J^{0} \cap\left(\mathcal{A}_{-} \cup \mathcal{A}_{+}\right)$. 'his proves our claim.

\section{. Domains of attraction}

this section we want to determine the domain of attraction of a generic $\omega$-limit set making $e$ of the existence of an invariant order resolution established in Section 2. More precisely, $X$ and $\Phi$ satisfy $(X)$ and $(\Phi)$, and let $X_{0}$ denote the set of all $x \in X$ with relatively mpact orbit. Given a totally invariant, compact, nonempty subset $W$ of $X$, the set

$$
\mathcal{D}(W)=\left\{x \in X_{0}: \omega(x) \subset W\right\}
$$

:alled the domain of attraction of $W$. Observe that if $W$ is connected, then so is $\mathcal{D}(W)$, ause every orbit is connected.

To complete our stability classification of a lower and/or upper approximable point $X$ from Section 3 we denote by $\mathcal{S}_{1 / 2}=\mathcal{S}_{-} \cup \mathcal{S}_{+}$the set of all $\omega$-semistable points, by $=U_{-} \cap U_{+}$the set of all $\omega$-biunstable points, and by $\mathcal{A}_{1 / 2}=\mathcal{A}_{-} \cup \mathcal{A}_{+}$the set of all nptotically w-semistable points.

Given a set $Y \subset X$, we denote by

$$
\partial_{-} Y=\left\{x \in \partial Y: x<y_{n} \in Y \text { for some sequence } y_{n} \longrightarrow x\right\}
$$

lower boundary of $Y$, and by

$$
\partial_{+} Y=\left\{x \in \partial Y: x>y_{n} \in Y \text { for some sequence } y_{n} \longrightarrow x\right\}
$$

:pper boundary of $Y$. Observe that, when $Y$ is open and order-convex, we have

$\exists_{-} Y=\{x \in \partial Y:[[x, \infty]] \cap Y \neq 0\}$ and $\partial_{+} Y=\{x \in \partial Y:[[-\infty, x]] \cap Y \neq \emptyset\}$.

d, if $x \in \partial Y$ and $x<y_{n} \in Y$, then also $x<y_{n} \ll y_{n}^{\prime}$ for some $y_{n}^{\prime} \in Y$. On the other if $x \in \partial Y$ and $x \ll y$ for some $y \in Y$, choose $y_{n} \in[[x, y]]$ with $y_{n} \longrightarrow x$, and also $Y$ with $x_{n} \longrightarrow x$ and $x_{n} \ll y_{n}$; then $y_{n} \in Y$ by the order-convexity of $Y$. Analogously $Y$.

inally, we denote by

$$
\operatorname{Fr}(Y)=\partial Y \backslash\left(\partial_{-} Y \cup \partial_{+} Y\right)
$$

me of $Y$. 
Next we introduce the following notation: $\mathcal{M}_{-}\left(\mathcal{M}_{+}\right.$, respectively) denotes the set of all $x \in X$ satisfying $\Phi, x>\Phi_{t} x\left(\Phi, x<\Phi_{t} x\right)$ for some $s, t \in R_{+}^{1}, s<t$. We set $\mathcal{M}=\mathcal{M}_{-} \cup \mathcal{M}_{+}$. Several important properties of these sets are stated in the following lemma:

Lemma 4.1. Let $X$ and $\Phi$ satisfy $(X)$ and $(\Phi)$, and let $X$ be strongly ordered. Then both $\mathcal{M}_{-}$and $\mathcal{M}_{+}$are order-open in $X$, and $\mathcal{M}_{-} \cap \mathcal{M}_{+}=\emptyset$. In particular, if $\Gamma$ is an invariant order resolution of $X$, then $\cup\{H=A \cap B:(A, B) \in \Gamma\}=X \backslash \mathcal{M}$ is order-closed.

Proof. Let $x \in \mathcal{M}_{-}$, i.e., $\Phi_{,} x>\Phi_{t} x$ for some $s<t$. Since $\Phi_{r}, \tau>0$, is strongly increasing and continuous, we have $\left.\Phi_{a+1} y \gg \Phi_{t+1} y, y \in[\| a, b]\right]$, for some $a, b \in X$ with $a \ll x \ll b$. Thus, $\|[a, b]] \subset \mathcal{M}_{-}$, and so $\mathcal{M}_{-}$is order-open. Analogously, $\mathcal{M}_{+}$is order-open. Finally, $\mathcal{M}_{-} \cap \mathcal{M}_{+}=0$ is the Non-oscillation principle of Hirsch [20, Lemma 6.1].

A set $Y \subset X$ is called order-connected if every pair $x, y \in Y, x<y$, is contained in a simply ordered, compact arc $J \subset Y$. Observe that if $X$ is strongly ordered and orderconnected, then every open order interval $[[a, b]]$ in $X, a, b \in X$, is arcwise connected, and in particular, $\hat{X}$ is locally arcwise connected.

Our first result is an analogue of a theorem from Takác [45, Thm. 5.1] stated for discrete-time semigroups.

Theorem 4.2. Let $X$ and $\Phi$ satisfy $(X)$ and $(\Phi)$, and let $X$ be locally connected, strongly ordered and order-connected. Let $\Gamma$ be any invariant order resolution of $X$. Assume that $Y$ is a component of $\mathcal{M}_{-}$satisfying $Y \subset X_{0}$. Then we have the following statements:

(a) The sets $Y$ and $X \backslash Y$ are invariant.

(b) There exists $p=\mathcal{E} \cap \partial_{-} Y$ such that

$$
\Phi_{t} x \longrightarrow p \text { as } t \longrightarrow \infty, \text { for every } x \in Y \cup\left(\partial_{-} Y \cap X_{0}\right) \text {. }
$$

(c) $p \ll \omega(x)$ for every $x \in \partial_{+} Y \cap X_{0}$.

(d) $\operatorname{Fr}(Y) \cap \mathcal{S}_{1 / 2}=0$; in particular, every $x \in \operatorname{Fr}(Y)$ which is lower (upper, resp.) approximable satisfies $x \in \mathcal{U}_{-}$and $\omega_{-}(x) \ll p\left(x \in U_{+}\right.$and $\left.p \ll \omega_{+}(x)\right)$, where $\omega_{-}(x)$ $\left(\omega_{+}(x)\right)$ is a single equilibrium provided some point $w \in \omega(x)$ is lower (upper) approximable.

An analogous theorem holds if $Y$ is a component of $\mathcal{M}_{+}$. Every component of $\mathcal{M}$ is a component of either $\mathcal{M}_{-}$or else $\mathcal{M}_{+}$.

Our proof of this theorem is based on the following lemma which also provides more information about the order structure of the sets $Y$ and $\partial Y$.

Lemma 4.3. Let $X$ and $\Phi$ satisfy $(X)$ and $(\Phi)$, and let $X$ be locally connected, strongly ordered and order-connected. Let $\Gamma$ be any invariant order resolution of $X$. Assume that 
$Y$ is a component of $\mathcal{M}$. Then either $Y \subset \mathcal{M}_{-}$or else $Y \subset \mathcal{M}_{+}$. Furthermore, there exist $\left(A_{1}, B_{1}\right),\left(A_{2}, B_{2}\right) \in \Gamma \cup\{(\theta, X),(X, \emptyset)\}$ such that $A_{1} \subset A_{2}$ and $Y$ is a component of $\operatorname{Int}\left(A_{2}\right) \cap \operatorname{Int}\left(B_{1}\right) \subset \mathcal{M}$. In particular, the following statements are valid:

(a) $Y$ is order-open and order-convex in $X$.

(b) $\partial_{-} Y \subset H_{1}=A_{1} \cap B_{1}, \partial_{+} Y \subset H_{2}=A_{2} \cap B_{2}$ and $\operatorname{Fr}(Y) \subset H_{1} \cap H_{2}$. Moreover, the sets $\partial_{-} Y$ and $\partial_{+} Y$ are open in $\hat{H}_{1}$ and $\hat{H}_{2}$, resp., whereas $\operatorname{Fr}(Y)=\partial Y \cap H_{1} \cap H_{2}$ is closed in $X$.

(c) All sets $Y, X \backslash Y, \partial_{-} Y, \partial_{+} Y$ and $\operatorname{Fr}(Y)$ are invariant.

PROOF. By Lemma 4.1, both $\mathcal{M}_{-}$and $\mathcal{M}_{+}$are order-open in $X$ and satisfy $\mathcal{M}=$ $\mathcal{M}_{-} \cup \mathcal{M}_{+}$and $\mathcal{M}_{-} \cap \mathcal{M}_{+}=0$. Since $Y$ is a component of $\mathcal{M}_{\text {, it must also be a component }}$ of either $\mathcal{M}_{-}$or else $\mathcal{M}_{+}$.

Next we set $\tilde{\Gamma}=\Gamma \cup\{(\theta, X),(X, \emptyset)\}$ and define

$$
\tilde{\Gamma}^{1}=\{(A, B) \in \tilde{\Gamma}: Y \subset \operatorname{Int}(B)\} \quad \text { and } \quad \tilde{\Gamma}^{2}=\{(A, B) \in \tilde{\Gamma}: Y \subset \operatorname{Int}(A)\}
$$

Observe that both $\tilde{\Gamma}^{1}$ and $\tilde{\Gamma}^{2}$ are nonempty and satisfy $A^{1} \subset A^{2}$ whenever $\left(A^{i}, B^{i}\right) \in \tilde{\Gamma}^{i}$, $i=1,2$. Now define

$$
\begin{aligned}
& A_{1}^{\prime}=\cup\left\{A:(A, B) \in \tilde{\Gamma}^{1}\right\}, \quad B_{1}^{\prime}=\cap\left\{\operatorname{Int}(B):(A, B) \in \tilde{\Gamma}^{1}\right\} \\
& A_{2}^{\prime}=\cap\left\{\operatorname{Int}(A):(A, B) \in \tilde{\Gamma}^{2}\right\}, \quad B_{2}^{\prime}=\cup\left\{B:(A, B) \in \tilde{\Gamma}^{2}\right\}
\end{aligned}
$$

and set

$$
\mathcal{A}_{i}=\mathrm{Cl}\left(A_{i}^{\prime}\right), B_{i}=\mathrm{Cl}\left(B_{i}^{\prime}\right) \text { for } i=1,2, \quad \text { and } \quad Z=\operatorname{Int}\left(A_{2}\right) \cap \operatorname{Int}\left(B_{1}\right) .
$$

Using the same arguments as in the proof of Theorem 2.2, where the rôle of our present set $Y$ is played by the point $x$, we deduce that $\left(A_{i}, B_{i}\right) \in \tilde{\Gamma}^{i}$ and $A^{1} \subset A_{1} \subset A_{2} \subset A^{2}$ whenever $\left(A^{i}, B^{i}\right) \in \tilde{\Gamma}^{i}$, for $i=1,2$. Obviously $Y \subset Z$. We claim that also

$$
\tilde{\Gamma}=\tilde{\Gamma}^{1} \cup \tilde{\Gamma}^{2} .
$$

Suppose not; then $A_{1} \subset A \subset A_{2}$ for some $(A, B) \in \tilde{\Gamma} \backslash\left(\tilde{\Gamma}^{1} \cup \tilde{\Gamma}^{2}\right)$ which forces $(A, B) \in \Gamma$. Thus $Y \cap A \neq \emptyset$ and $Y \cap B \neq \emptyset$, whence $Y \cap \operatorname{Int}(A) \neq \emptyset$ and $Y \cap \operatorname{Int}(B) \neq \emptyset$, since $Y \cap H=\emptyset$ where $H=A \cap B$. But this means that $Y$ is contained in the union of two separated sets $\operatorname{Int}(A)$ and $\operatorname{Int}(B)$ and intersects both of them; a contradiction to $Y$ is connected. So (1) is valid.

From (1) we obtain $Z \subset \mathcal{M}$, and so $Y$ is also a component of $Z$. Now we are ready to prove the remaining statements:

(a) Since both $\operatorname{Int}\left(A_{2}\right)$ and $\operatorname{Int}\left(B_{1}\right)$ are order-convex and order-open, so are $Z$ and $Y$. because $Z \subset X$ is order-connected and $Y$ is a component of $Z$. 
(b) Since $Y$ is a component of the open set $\mathcal{M} \subset X$, we have $\partial Y \subset X \backslash \mathcal{M}$. Moreover, $Y \subset Z$ and (1) force $\partial Y \subset H_{1} \cup H_{2}$. Now fix any $x \in \partial_{-} Y$; hence $x \in \partial Y$ and $x \ll y$ for some $y \in Y$. Then $x \notin B_{2}$ since $y \notin B_{2}$ and $B_{2}$ is upper closed. Consequently $x \in H_{1}$. Observe that also $\operatorname{Int}\left(B_{1}\right) \cap[[-\infty, y]] \subset Y \subset \operatorname{Int}\left(B_{1}\right)$ which, in combination with $H_{1}=\partial B_{1}=\partial_{-}\left(\operatorname{Int}\left(B_{1}\right)\right)$, implies $\left.H_{1} \cap \|[-\infty, y]\right] \subset \partial_{-} Y$. Hence, $\partial_{-} Y$ is an open subset of $\hat{H}_{1}$. Analogously, $\partial_{+} Y$ is open in $\hat{H}_{2}$.

Finally, fix any $x \in \operatorname{Fr}(Y)$; hence $x \in \partial Y \backslash\left(\partial_{-} Y \cup \partial_{+} Y\right)$, and we may assume also $x \in H_{1}$; the case $x \in H_{2}$ is analogous. Suppose $x \notin H_{2}$. Then $x \in \operatorname{Int}\left(A_{2}\right)$, and there exist $z \in Z \backslash Y$ with $x \ll z$ and also $y \in Y$ with $y \ll z$. Since $X$ is order-connected, there exists a simply ordered, compact arc $J \subset X$ with endpoints $y$ and $z$. The order-convexity of $Z$ forces $J \subset Z \subset \mathcal{M}$. But $y \in J \cap Y$ and $Y$ is a component of $Z$ imply $J \subset Y$; in particular, $z \in Y$ contradicts our choice of $z \in Z \backslash Y$. It follows that $x \in H_{2}$, and so $\operatorname{Fr}(Y) \subset \partial Y \cap H_{1} \cap H_{2}$. The inclusion $\partial Y \cap H_{1} \cap H_{2} \subset \operatorname{Fr}(Y)$ is an easy consequence of $Y \subset Z$. We conclude that $\operatorname{Fr}(Y)=\partial Y \cap H_{1} \cap H_{2}$ is closed in $X$.

(c) Since $\Phi\left(\mathbf{R}_{+}^{1} \times Y\right)=U\left\{\Phi_{t}(Y): t \in \mathbf{R}_{+}^{1}\right\}$ is a connected subset of $\mathcal{M}$, we must have $\Phi\left(\mathbf{R}_{+}^{1} \times Y\right)=Y$, i.e., $\Phi_{t}(Y) \subset Y$ for all $t \in \mathbf{R}_{+}^{1}$. Similarly, since $Y$ is a component of $\mathcal{M}$, the set $Y^{\#}=\left\{x \in X: \mathcal{O}^{+}(x) \cap Y \neq \theta\right\}$ is connected with $Y \subset Y^{\#} \subset \mathcal{M}$, and consequently $Y^{\#}=Y$, i.e., $\Phi_{t}(X \backslash Y) \subset X \backslash Y$ for all $t \in \mathbf{R}_{+}^{1}$. Furthermore, $\partial Y \subset H_{1} \cup H_{2}$ and $\Phi_{1}(\partial Y) \subset$ $\mathrm{Cl}(Y)$ imply $\Phi_{i}\left(\partial Y \cap H_{i}\right) \subset \partial Y \cap H_{i}, i=1,2$. This proves that $\operatorname{Fr}(Y)=\partial Y \cap H_{1} \cap H_{2}$ is invariant under $\Phi_{1}$. Now fix any $x \in \partial_{-} Y$, i.e., $x \in \partial Y$ and $x \ll y$ for some $y \in Y$. Then $\Phi_{t} x \ll \Phi_{t} y$, by the strong monotonicity of $\Phi_{t}, t>0$, and also $\Phi_{t} x \in \partial Y$ and $\Phi_{t} y \in Y$. Consequently $\Phi_{t} x \in \partial_{-} Y$ as desired. We have proved that $\partial_{-} Y$ is invariant under $\Phi_{t}$, and analogously for $\partial_{+} Y$. This completes the proof of our lemma.

Proof of Theorem 4.2. (a) We have $\Phi_{t}(Y) \subset Y$ and $\Phi_{t}(X \backslash Y) \subset X \backslash Y, t>0$, by Lemma $4: 3$ (c).

(b) Since $Y \subset \mathcal{M}_{-} \cap X_{0}$, we can find $y \in Y$ such that $\Phi_{r} y<y$ for some $\tau \in R_{+}^{1} \backslash\{0\}$, and $\Phi_{t} y \longrightarrow p$ as $t \longrightarrow \infty$, for some $p \in \mathcal{E}$, by Prop. 3.1. Since also $p \ll y$ and $p \notin \mathcal{M}$ by Prop. 3.2, we obtain $p \in \partial_{-} Y$. Hence, the set

$$
Z=\left\{x \in Y: \Phi_{t} x \longrightarrow p \text { as } t \longrightarrow \infty\right\}
$$

is nonempty. We claim that $Z$ is both open and closed in $Y$. We first show that $Z$ is lower and upper closed in $Y$.

Pick any $x \in Z$ and $y \in Y$ with $y \leq x$. Let $H_{i}=A_{i} \cap B_{i}, i=1,2$, be as in Lemma 4.3. Then $\omega(y) \leq \omega(x)=\{p\}$ in $H_{1}$ and $H_{1}$ unordered imply $\omega(y)=\{p\}$, and consequently $y \in Z$. Hence, $Z$ is lower closed in $Y$.

Now pick any $x \in Z$ and $y \in Y$ with $x \leq y$. Then $y \in \mathcal{M}_{-}$shows that $\omega(y)$ is a single equilibrium and $\omega(y) \subset \partial_{-} Y$. Since also $\{p\}=\omega(x) \leq \omega(y)$ in $H_{1}$, we have $\omega(y)=\{p\}$ again, and consequently $y \in Z$. Thus, $Z$ is also upper closed in $Y$. 
To show that $Z$ is open in $Y$ we choose any $x \in Z$. Since $Y$ is open in $X$, we can find $y \in Y$ such that $x \ll y$. But then $y \in Z$ because $Z$ is upper closed in $Y$, and consequently $x \in[[-\infty, y]] \cap Y \subset Z$ because $Z$ is lower closed in $Y$. So $Z$ is open in $Y$.

To show that $Z$ is closed in $Y$ we fix any $x \in \mathrm{Cl}(Z) \cap Y$. As above we can find $y \in Y$ with $x \ll y$. Then also $z \ll y$ for some $z \in Z$. Since $Z$ is upper and lower closed in $Y$, we arrive at $y \in Z$ and $x \in Z$. Hence, $Z$ is also closed in $Y$.

Finally, we must have $Z=Y$ since $Y$ is connected. In particular, if $x \in \partial_{-} Y \cap X_{0}$ then $x \ll y$ for some $y \in Y$, whence $\omega(x) \leq \omega(y)=\{p\}$ in $H_{1}$ which, in turn, forces $\omega(x)=\{p\}$ and also $\Phi_{t} x \longrightarrow p$ as $t \rightarrow \infty$. This finishes the proof of (b).

(c) Take any $x \in \partial_{+} Y \cap X_{0}$. Then $y \ll x$ for some $y \in Y$, whence $\omega(x) \subset H_{2}$ satisfies $\{p\}=\omega(y) \ll \omega(x)$, by Lemma $4.3(b)$.

(d) Fix any $x \in \operatorname{Fr}(Y)$. First assume that $x$ is lower approximable, i.e., there exists a sequence $x_{n} \in X, x_{n}<x$, such that $x_{n} \longrightarrow x$ and $\Phi$ is $\omega$-compact in $\left\{x, x_{1}, x_{2}, \cdots\right\}$. Then $\Phi_{1} x_{n} \ll \Phi_{1} x \in \operatorname{Fr}(Y)$ shows that $\Phi_{1} x_{n} \ll y_{n}$ for some $y_{n} \in Y$, and therefore $\omega\left(x_{n}\right) \leq \omega\left(y_{n}\right)=p$ by Part (b), where $n=1,2, \cdots$. We claim $p \notin \omega\left(x_{n}\right)$ for any $n \in N$. Suppose not; then $p \in \omega\left(x_{n}\right)$ for some $n \in N$, whence $\omega\left(x_{n}\right)=p$ because $\omega\left(x_{n}\right)$ is unordered by Prop 3.2. This implies $p \leq \omega(x)$ in $H_{1}$, and consequently $\omega(x)=p$ because $H_{1}$ is unordered. But $\omega(x) \subset \operatorname{Fr}(Y)$ since $\operatorname{Fr}(Y)$ is closed and invariant, by Lemma 4.3 (b,c), and so $p \in \operatorname{Fr}(Y)$. This contradicts $p \in \partial_{-} Y$; hence $p \notin \omega\left(x_{n}\right)$ must hold for every $n \in N$. Moreover, we have $\omega\left(x_{n}\right) \ll p$. We apply Prop. 3.6 to conclude that $\omega_{-}(x) \ll p$, where $\omega_{-}(x)$ is a single equilibrium provided $z<y<w$ for some $w \in \omega(x), z \in \omega_{-}(x)$ and $y \in X_{0}$ which is the case if $w$ is lower approximable. Observe that the hypothesis $x \in \mathcal{U}_{-}$in Prop. 3.6 is satisfied, since otherwise $\omega_{-}(x)=\omega(x)$ combined with $\omega\left(x_{n}\right) \ll p$ would imply $\omega(x) \leq p$ in $H_{1}$, and consequently $p=\omega(x) \subset \operatorname{Fr}(Y)$ as above which is a contradiction to $p \in \partial_{-} Y$ again. In particular, $x \notin \mathcal{S}_{-}$.

Now assume that $x$ is upper approximable, i.e., there exists a sequence $x_{n} \in X, x_{n}>x$, such that $x_{n} \longrightarrow x$ and $\Phi$ is $\omega$-compact in $\left\{x, x_{1}, x_{2}, \cdots\right\}$. In analogy with the previous case we obtain $p \leq \omega\left(x_{n}\right) \subset B_{2}$ since $x_{n} \in B_{2}$. Then $p \in \partial_{-} Y \subset \operatorname{Int}\left(A_{2}\right)$ implies $p \notin \omega_{+}(x) \subset B_{2}$ and $p \ll \omega_{+}(x)$. We apply Prop. 3.6 again, with $x \in \mathcal{U}_{+}$, to obtain the desired conclusion. The hypothesis $x \in U_{+}$is satisfied, since otherwise $\omega_{+}(x)=\omega(x)$ would imply $p \ll \omega(x)$ in $H_{1}$, a contradiction to $H_{1}$ is unordered. In particular, $x \notin \mathcal{S}_{+}$. We have proved also $\operatorname{Fr}(Y) \cap \mathcal{S}_{1 / 2}=0$. The proof of Theorem 4.2 is now complete.

A very useful consequence of Theorem 4.2 is the following extension of Proposition 3.6 (Discontinuity principle) which provides an interesting description of the set $U_{-}\left(U_{+}\right.$, resp.) of all lower (upper) w-unstable points.

Proposition 4.4. Let $X, \Phi$ and $\Gamma$ be as in Theorem 4.2. Assume that every $x \in X$ has relatively compact orbit. Then we have

$$
U_{-}=\cup\left\{\partial_{+} Y: Y \text { is a component of } \mathcal{M}_{-}\right\} \text {, }
$$


and the following statement is valid:

Suppose $x \in \mathcal{U}_{-}$, and let $Y$ be the component of $\mathcal{M}_{-}$such that $x \in \partial_{+} Y$. Then there exists $a \in Y, a \ll x$, such that every $y \in X$ with $a \leq y<x$ satisfies also $y \in Y$ and $\omega(y)=\omega_{-}(x)=\{p\}$. Here $p \in \mathcal{E}$ is as in Theorem 4.2.

A corresponding result holds for $\mathcal{M}_{+}$and $U_{+}$.

PrOOF. First consider any component $Y$ of $\mathcal{M}_{-}$and any $x \in \partial_{+} Y$. Let $\left(A_{i}, B_{i}\right)$ and $H_{i}=A_{i} \cap B_{i}, i=1,2$, be as in Lemma 4.3. Then $Y$ is a component of $W=\operatorname{Int}\left(A_{2}\right) \cap \operatorname{Int}\left(B_{1}\right)$, and $x \in H_{2}$. Since $x \in \partial_{+} Y$, we can find $a \in Y$ with $a \ll x$. Lemma 4.3(a) shows that every $y \in X, a \leq y<x$, satisfies also $y \in Y$. Furthermore, we have $\omega(y)=\{p\} \ll \omega(x)$ for every $y \in Y$, by Theorem 4.2(b,c). We conclude that $x \in \mathcal{U}_{-}$and $\omega_{-}(x)=\{p\}$ as desired.

Now consider any $x \in \mathcal{U}_{-}$. Set

$$
Y_{0}=\left\{y \in X: p<y<q \text { for some } p \in \omega_{-}(x) \text { and } q \in \omega(x)\right\} .
$$

Obviously $Y_{0} \neq 0$, and therefore we may apply Prop. 3.6 to obtain $\omega(y)=\omega_{-}(x)=\{p\}$ for every $y \in Y_{0}$, where $p \in \mathcal{E}$. It is now easy to see that $Y_{0} \subset \mathcal{M}_{-}$. Moreover, the orderconnectedness of $X$ implies that $Y_{0}$ is connected, and so $Y_{0} \subset Z$ where $Z$ is a component of $\mathcal{M}_{-}$. Let $\tilde{\Gamma}=\Gamma \cup\{(\theta, X),(X, \theta)\}$. By Lemma 4.3 with $Z$ in place of $Y$, there exist $\left(A_{i}, B_{i}\right) \in \tilde{\Gamma}, i=1,2$, such that $Z$ is a component of $W=\operatorname{Int}\left(A_{2}\right) \cap \operatorname{Int}\left(B_{1}\right) \subset \mathcal{M}$. By Theorem $4.2(\mathrm{~b}, \mathrm{c})$ we have

$$
\omega_{-}(x)=\{p\} \subset \partial_{-} Z \subset H_{1} \quad \text { and } \quad \omega(x) \subset \partial_{+} Z \subset H_{2},
$$

where $H_{j}=A_{j} \cap B_{j}, j=1,2$. We claim that $x \in \operatorname{Int}\left(B_{1}\right) \cap H_{2}$.

Suppose not; then we can have only the following alternatives:

(i) $x \in A_{1}$. Then also $\omega(x) \subset A_{1}$. Hence, $\omega_{-}(x) \ll \omega(x)$ implies $\omega_{-}(x) \subset \operatorname{Int}\left(A_{1}\right)$ which contradicts $\omega_{-}(x)=\{p\} \subset H_{1}$. We have verified $x \in \operatorname{Int}\left(B_{1}\right)$.

(ii) $x \in W=\operatorname{Int}\left(A_{2}\right) \cap \operatorname{Int}\left(B_{1}\right)$. Then either $x \in \mathcal{M}_{-}$or else $x \in \mathcal{M}_{+}$. In either case $x \in \mathcal{A}=\mathcal{A}_{-} \cap \mathcal{A}_{+}$by Theorem 4.2(b), a contradiction to $x \in \mathcal{U}_{-}$.

(iii) $x \in \operatorname{Int}\left(B_{2}\right)$. Then $\omega_{-}(x) \subset B_{2}$. Hence, $\omega_{-}(x) \ll \omega(x)$ implies $\omega(x) \subset \operatorname{Int}\left(B_{2}\right)$ which contradicts $\omega(x) \subset H_{2}$.

We have verified $x \in \operatorname{Int}\left(B_{1}\right) \cap H_{2}$. By Prop. 3.6 we can find $a \in X, a \ll x$, such that $\omega(y)=\{p\}$ for every $y \in X, a \leq y<x$. We may choose a with $a \in \operatorname{Int}\left(B_{1}\right)$; hence $a \in W$. Let $Y$ be the component of $\mathcal{M}$ containing $a$. Since $X$ is order-connected, there exists a simply ordered, compact arc $J \subset X$ with endpoints $a$ and $x$. Observe that $J \backslash\{x\} \subset W \subset \mathcal{M}$ forces $J \backslash\{x\} \subset Y$, whence $x \in \partial_{+} Y$. We claim also $Y \subset \mathcal{M}_{-}$. Indeed, if $Y \subset \mathcal{M}_{+}$then Theorem 4.2(b) entails $x \in \mathcal{A}_{-}$which contradicts $x \in \mathcal{U}_{-}$. We conclude that $x \in \partial_{+} Y$ for some component $Y$ of $\mathcal{M}_{\text {.. }}$. This completes our proof.

Even more can be said about the structure of the sets $\mathcal{U}_{-}$and $\mathcal{U}_{+}$when $X \subset V$ : 
Corollary 4.5. Let $X$ be an order-open and order-connected subset of a separable strongly ordered vector space $V$, and let $\Phi: R_{+}^{1} \times X \longrightarrow X$ satisfy $(\Phi)$. Assume that every $x \in X$ has relatively compact orbit in $X$. Then $\mathcal{M}$ has at most countably many components, and $U_{-}$is the union of at most countably many Lipschitz manifolds of codimension one in $\hat{V}$. Each of these manifolds has the form $\partial_{+} Y$, where $Y$ is any component of $\mathcal{M}_{-}$, and $\partial_{+} Y$ is an open subset of $\hat{H}$ where $H=A \cap B$ for some $(A, B) \in \Gamma$, any given invariant order resolution of $X$. Here $\hat{H}$ is a Lipschitz hypersurface in $\hat{V}$ as described in Proposition 1.3.

A corresponding result holds for $\mathcal{U}_{+}$.

In particular, if $V$ is a separable strongly ordered Banach space and $\mu$ is a Gaussian measure on $V$, then $\mu(\mathcal{U})=0$ where $\mathcal{U}=\mathcal{U}_{-} \cup \mathcal{U}_{+}$. If $V=\mathbf{R}^{N}$ then $U$ has zero Lebesgue measure, too.

The reader is referred to H-H. Kuo [25] for general facts about Gaussian measures in Banach spaces, and to Aronszajn [9] and Phelps [31] for descriptions of their null sets. Some additional details about null sets can be found in Hirsch [20, Lemma 7.7].

P ROOF of Corollary 4.5. We have $U_{-}=U\left\{\partial_{+} Y: Y\right.$ is a component of $\left.\mathcal{M}_{-}\right\}$by Prop. 4.4. Here each $\partial_{+} Y$ is an open subset of some $\hat{H}$ where $H=A \cap B$ for a suitable $(A, B) \in \Gamma$, by Lemma 4.3(b). The set $\hat{H}$ is a Lipschitz hypersurface in $\hat{V}$, by Prop. 1.3, which is homeomorphic to an open subset of a closed hyperplane in $\hat{V}$. It follows that $\partial_{+} Y$ is a Lipschitz manifold of codimension one in $\hat{V}$. Since $V$ is separable, the open set $\mathcal{M}$ can have at most countably many components which, of course, are open and belong to either $\mathcal{M}_{\text {- }}$ or else $\mathcal{M}_{+}$, by Lemma 4.1.

Our claim that $\mu(\mathcal{U})=0$ follows immediately from Hirsch [20, Lemma $7.7(a)]$.

To state our second result we need the following concept. Given a set $Y \subset X$, we denote by $\operatorname{Arcint}(Y)$ the set of all $x \in Y$ such that $x \in J$ for some simply ordered, open arc $J \subset Y$; we call it the arc interior of $Y$. Observe that $\operatorname{Int}(Y) \subset \operatorname{Arcint}(Y)$ provided $X$ is strongly ordered and order-connected. We say that $Y$ is $\operatorname{arc}$-open if $Y=\operatorname{Arcint}(Y)$. If, in addition, $T: X \longrightarrow X$ satisfies $(T)$, then $T$ is an arc-open mapping:

$$
Y \text { is arc-open } \Longrightarrow T(Y) \text { is arc-open. }
$$

(Warning: The arc-open subsets of $X$ do not necessarily define a topology on $X$, because the intersection of two simply ordered, open arcs in $X$ might be a singleton; take $X=\mathbf{R}^{2}$ as an example.)

We denote $\mathcal{N}=\operatorname{Arcint}(X \backslash \mathcal{M})$. Our second result is an analogue of a theorem from Takác $[45$, Thm. 5.7] stated for discrete-time semigroups.

Theorem 4.6. Let $X, \Phi$ and $\Gamma$ be as in Theorem 4.2. Ansume that every $x \in X$ has relatively compact orbit. Let $Y$ be a component of $\mathcal{N}^{\prime}$ such that $\Phi$ is w-compact in 
every simply ordered, compact arc $J \subset Y \cup \partial_{-} Y \cup \partial_{+} Y$. Then $Y$ and $X \backslash Y$ are invariant, $Y \subset \mathcal{S}=\mathcal{S}_{-} \cap \mathcal{S}_{+}$and precisely one of the following two alternatives holds provided every simply ordered, order-connected subset of $\mathcal{E}$ is separable in the relative topology from $X$ :

(a) $\omega\left(a_{1}\right) \neq \omega\left(a_{2}\right)$ for some simply ordered, compact are $L \subset Y$ with endpoints $a_{1}$ and $a_{2}$. In this case there exists a strictly increasing continuous path $P: I \longrightarrow X$ (with its image) contained in $\mathcal{E}$, for some nondegenerate interval $I \subset \mathbf{R}^{1}$, with the following property:

For every $x \in Y \cup \partial_{-} Y \cup \partial_{+} Y$ there exists a unique $\tau \in \mathcal{I}$ satisfying

$$
\Phi_{\imath} x \longrightarrow P(\tau) \text { in } X \text { as } t \longrightarrow \infty \text {. }
$$

(b) $\omega\left(a_{1}\right)=\omega\left(a_{2}\right)$ for every simply ordered, compact arc $L \subset Y$ with endpoints $a_{1}$ and $a_{2}$. In this case, for every $x_{0} \in Y$ and every sequence $t_{1}<t_{2}<\cdots$ in $\mathbf{R}_{+}^{1}$ satisfying $t_{k} \longrightarrow \infty$ and $\Phi_{t_{k}} x_{0} \longrightarrow w$ as $k \longrightarrow \infty$, for some $w \in X$, we have:

$$
\Phi_{t_{\star}} x \longrightarrow w \text { in } X \text { as } k \longrightarrow \infty,
$$

for every $x \in Y \cup \partial_{-} Y \cup \partial_{+} Y$. In particular, we have $\omega(x)=\omega\left(x_{0}\right) \subset \mathcal{E}$.

Furthermore, in both cases (a) and (b), the set $Y$ is order-convex and order-open in $X$. Finally, if $Z$ is another component of $\mathcal{N}$ such that $\Phi_{r}(Y) \cap Z \neq 0$ for some $\tau \in R_{+}^{1}$, then $\Phi_{r}(Y) \subset Z=Y$.

PROOF. Since $\Phi$ is continuous and strongly increasing, the set $\Phi\left(\mathbf{R}_{+}^{1} \times Y\right)=\cup\left\{\Phi_{t}(Y)\right.$ : $\left.t \in \mathbf{R}_{+}^{\mathbf{1}}\right\}$ is connected and arc-open and satisfies $Y \subset \boldsymbol{\Phi}\left(\mathbf{R}_{+}^{\mathbf{1}} \times Y\right) \subset \mathcal{N}$. But $Y$ is a component of $\mathcal{N}$ forces $\Phi\left(R_{+}^{1} \times Y\right)=Y$, i.e., $Y$ is invariant.

To prove the invariance of $X \backslash Y$ we need the fact that $Y$ is order-open in $X$ which will be verified in the proofs of Alt. (a) and (b) below. We combine this fact with the order-continuity of each $\Phi_{\mathbf{l}}, t \in \mathbf{R}_{+}^{1}$, to conclude that also

$$
Y^{\#}=\left\{x \in X: \mathcal{O}^{+}(x) \cap Y \neq \theta\right\}=\cup_{t \geq 0} \Phi_{t}^{-1}(Y)
$$

is order-open. Consequently, $Y \subset Y^{\#} \subset \mathcal{N}$ because $X$ is order-connected. Moreover, $Y^{\#}$ is connected since $Y$ and every orbit are connected. Thus, $Y$ is a component of $\mathcal{N}$ forces $Y^{\#}=Y$, i.e., $X \backslash Y$ is invariant.

Suppose there exists $x \in Y \backslash \mathcal{S}$. Hence, $x \in J$ for some simply ordered, open arc $J \subset Y$. We may assume $x \in \mathcal{U}_{-}$, the case $x \in \mathcal{U}_{+}$being analogous. By Prop. 4.4 we have also $x \in \partial_{+} Y^{\prime}$ for a suitable component $Y^{\prime}$ of $\mathcal{M}$, and there exists $a^{\prime} \in Y^{\prime}, a^{\prime} \ll x$, such that every $y^{\prime} \in X$ with $a^{\prime} \leq y^{\prime}<x$ satisfies also $y^{\prime} \in Y^{\prime}$. In particular, $J \cap Y^{\prime} \neq \emptyset$ which contradicts $J \subset Y \subset X \backslash \mathcal{M}$. We conclude that $Y \subset \mathcal{S}$ as claimed. Next we prove the alternatives (a) and (b).

(a) Assume $\omega\left(a_{1}\right) \neq \omega\left(a_{2}\right)$ for some simply ordered, compact arc $L \subset Y$ with endpoints $a_{1}$ and $a_{2}\left(a_{1}<a_{2}\right)$. Then we may apply Prop. 3.10 to obtain the existence of an $\omega$-limit fibre $F_{L}$ of $L$ such that $F_{L} \subset \mathcal{E}$ and for every $x \in L$ :

$$
\Phi_{1} x \longrightarrow w \in F_{L} \quad \text { as } t \longrightarrow \infty .
$$


Let $\mathcal{F}$ denote the collection of all simply ordered, compact arcs $F \subset \mathcal{E}$ containing $F_{L}$. We claim that $F_{1}, F_{2} \in \mathcal{F} \Longrightarrow F_{1} \cup F_{2} \in \mathcal{F}$. Indeed, pick any $F_{1}, F_{2} \in \mathcal{F}$ and set

$$
\alpha_{i}=\min \left(F_{i}\right) \text { and } \beta_{i}=\max \left(F_{i}\right) \text { for } i=1,2 \text {. }
$$

By Takáč $\left[43\right.$, Thm. 1.3] or [45, Theorem 2.5(a)], the order interval $\left[\alpha_{i}, \beta_{i}\right]$ is attracted by the set $F_{i}, i=1,2$. Hence, $F_{1} \cap\left[\alpha_{2}, \beta_{2}\right] \subset F_{2}$ and $F_{2} \cap\left[\alpha_{1}, \beta_{1}\right] \subset F_{1}$. These inclusions force either $\alpha_{1} \leq \alpha_{2}$ or else $\alpha_{1}>\alpha_{2}$, and either $\beta_{1} \leq \beta_{2}$ or else $\beta_{1}>\beta_{2}$, and consequently, also $F_{1} \cup F_{2} \in \mathcal{F}$. From the properties of $\mathcal{F}$ we deduce that the union $P^{*}=\cup\{F: F \in \mathcal{F}\}$ is simply ordered and order-connected. Obviously $P^{*} \subset \mathcal{E}$, and so $P^{*}$ is separable by hypothesis. It follows from Wilder [47, Chap. I, Thm. 11.12] that $P^{*}=\operatorname{Image}(P)$ where $P: \mathcal{I} \longrightarrow X$ is a strictly increasing continuous path defined in a nondegenerate interval $\mathcal{I} \subset \boldsymbol{R}^{\mathbf{1}}$. We claim:

$P^{*}$ attracts $Y$. Proof: Set $Y^{*}=\left\{y \in Y: \Phi_{\imath} y \rightarrow p \in P^{*}\right.$ as $\left.t \rightarrow \infty\right\}$.

Since $Y$ is connected, it suffices to show that $Y^{\bullet}$ is both open and closed in $Y$. Observe that $L \subset Y^{*}$ by our construction of $P^{*}$. Moreover, the entire order interval $\left[a_{1}, a_{2}\right]$ is attracted by $P^{*}$, as a simple consequence of Takác [43, Thm. 1.3] or [45, Theorem 2.5(a)].

Pick any $y \in Y^{*}$. Hence $y \in J^{\circ}$ for some simply ordered, compact arc $J \subset Y$ with endpoints $a<b$, where $J^{\circ}=J \backslash\{a, b\}$. Then either $\omega(x)=\omega(y)=p \in P^{*}$ for all $x \in J$, or else there exists an $\omega$-limit fibre $F_{J}$ of $J$ such that $F_{J} \subset \mathcal{E}$, by Prop. 3.3 and 3.10. Since $p \in F_{J} \cap P^{*} \neq \emptyset$, our definition of $P^{*}$ implies $F_{J} \subset P^{*}$. Thus $P^{*}$ attracts the order interval $[a, b]$, where $a<y<b$. Using $\Phi_{1} a \ll \Phi_{1} y \ll \Phi_{1} b$ we can find $u, v \in X$ such that $u \ll y \ll v$ and $\Phi_{1} a \leq \Phi_{1} u \ll \Phi_{1} v \leq \Phi_{1} b$. We conclude that $[[u, v]]$ is attracted by $P^{*}$. In particular, $Y^{*}$ is open in $Y$.

Now consider any $y \in \mathrm{Cl}\left(Y^{*}\right) \cap Y$. Using the same arguments as above we can find $u, v \in X$ such that $u \ll y \ll v$ and $[[u, v]]$ is attracted by either $\omega(y)$, or else by an $\omega$-limit fibre $F_{J} \subset \mathcal{E}$. Choosing any $y^{\prime} \in Y^{*} \cap[[u, v]] \neq \emptyset$ we arrive at either $\omega(y)=\omega\left(y^{\prime}\right)=p^{\prime} \in P^{*}$, or else $F_{J} \subset P^{*}$. In either case $\omega(y)=p \in P^{*}$, and so $y \in Y^{*}$. Thus, $Y^{*}$ is open and closed in $Y$, and $Y^{*} \neq \emptyset$, whence $Y^{*}=Y$ as claimed.

To prove that $Y$ is order-convex we fix arbitrary $u, v \in Y$ with $u<v$. As above we apply Takác $[43$, Thm. 1.3] or $[45$, Theorem $2.5(\mathrm{a})]$ to conclude that the entire order interval $[u, v]$ is attracted by either a simply ordered, compact arc or a singleton $P^{\prime} \subset P^{*}$. In particular, $\Phi$ is $\omega$-compact in $[u, v]$. Combining these facts with Prop. 3.6 and the orderconnectedness of $X$ we arrive at $u \in S_{+}, v \in \mathcal{S}_{-}$and $(u, v) \subset \mathcal{S}$ where $(u, v)=\{u, v\} \backslash\{u, v\}$. Now choose any $x \in(u, v)$. Hence, $x \in J^{\circ}$ for some simply ordered, compact arc $J \subset[u, v]$ with endpoints $u$ and $v$, where $J^{\circ}=J \backslash\{u, v\}$. We claim $J^{\circ} \subset X \backslash \mathcal{M}$. Suppose not, i.e., there exists $y \in J^{\circ} \cap \mathcal{M} \neq 0$. Let $Y^{\prime}$ denote the component of $\mathcal{M}$ containing $y$, and set $K=J \cap \mathrm{Cl}\left(Y^{\prime}\right)$. By Lemma 4.3, $K$ is a simply ordered, compact arc with endpoints $u^{\prime}$ and $v^{\prime}$, and $K^{\circ}=K \backslash\left\{u^{\prime}, v^{\prime}\right\} \subset Y^{\prime}$. Since $u, v \in \mathcal{N}=\operatorname{Arcint}(X \backslash \mathcal{M})$, we must have $u^{\prime}$, $v^{\prime} \in J^{\circ} \subset \mathcal{S}$. Also $u^{\prime} \in \partial_{-} Y^{\prime}$ and $v^{\prime} \in \partial_{+} Y^{\prime}$ provided $u^{\prime}<v^{\prime}$. It follows that $\partial_{-} Y^{\prime} \cap \mathcal{S} \neq \emptyset$ 
and $\partial_{+} Y^{\prime} \cap S \neq \theta$, a contradiction to Prop. 4.4. We conclude that $J^{\circ} \subset X \backslash \mathcal{M}$ as claimed. Consequently $x \in \mathcal{N}$, and so $x \in Y$, because $J \subset Y$. We have proved that $Y$ is order-convex.

To prove that $Y$ is order-open in $X$ we fix any $y \in Y$. Hence, $y \in J^{\circ}$ for some simply ordered, compact arc $J \subset Y$ with endpoints $a<b$, where $J^{\circ}=J \backslash\{a, b\}$. By the order-convexity of $Y$, the entire order interval $[a, b]$ is attracted by either a simply ordered, compact arc or a singleton $P^{\prime} \subset P^{\bullet}$. Obviously, the same is true of $\left[\Phi_{1} a, \Phi_{1} b\right]$, as $Y$ is invariant. Since $\Phi_{1} a \ll \Phi_{1} y \ll \Phi_{1} b$ we can find $u, v \in X$ such that $u \ll y \ll v$ and $\Phi_{1} a \ll \Phi_{1} u \ll \Phi_{1} v \ll \Phi_{1} b$. From $\Phi_{1}[u, v] \subset Y$ we deduce that $[u, v] \subset X \backslash \mathcal{M}$, and consequently also $\{[u, v]] \subset Y$ by the order-connectedness of $X$ combined with $y \in\{[u, v]\} \cap Y$. We conclude that $Y$ is also order-open.

Now we are ready to prove that $P^{*}$ attracts also $\partial_{-} Y$ and $\partial_{+} Y$. Both cases are analogous; we prove only

$P^{*}$ attracts $\partial_{-} Y$. Proof: Pick any $x \in \partial_{-} Y$. Since $Y$ is open and order-convex, there exists a sequence $x_{1} \gg x_{2} \gg \cdots \gg x$ satisfying $x_{n} \in Y$ and $x_{n} \longrightarrow x$. Hence, we can construct a simply ordered, compact arc $J \subset X$ with endpoints $x$ and $x_{1}$ such that $x_{n} \in J$, $n \in N$. We have $J \backslash\{x\} \subset Y$ by the order-convexity of $Y$. Then $\Phi$ is $\omega$-compact in $J$ by hypothesis, and consequently, either $x \in \mathcal{S}_{+}$or else $x \in \mathcal{U}_{+}$. The case $x \in \mathcal{U}_{+}$is easily excluded by Prop. 4.4 and $Y \subset \mathcal{S} \cap(X \backslash \mathcal{M})$; so $x \in \mathcal{S}_{+}$. In particular, the sequence $\omega\left(x_{n}\right)=p_{n} \in P^{*}$ has a limit $p \in X$ which satisfies also $p_{n} \searrow p$ and $\omega(x)=\omega_{+}(x)=p$. Our definition of $P^{*}$ forces $p \in P^{*}$. We have proved that $P^{*}$ attracts $\partial_{-} Y$.

Finally, let $Z$ be another component of $\mathcal{N}$ such that $\Phi_{r}(Y) \cap Z \neq 0$ for some $\tau \in \mathbf{R}_{+}^{1}$. Since $\Phi_{r}(Y) \subset Y$, we have also $Y \cap Z \neq 0$. It follows that $\Phi_{r}(Y) \subset Z=Y$ as desired.

(b) Assume $\omega\left(a_{1}\right)=\omega\left(a_{2}\right)$ for every simply ordered, compact arc $L \subset Y$ with endpoints $a_{1}$ and $a_{2}\left(a_{1}<a_{2}\right)$. Now fix any $x_{0} \in Y$ and any sequence $t_{1}<t_{2}<\cdots$ in $R_{+}^{1}$ satisfying $t_{k} \rightarrow \infty$ and

$$
\Phi_{t_{\star}} x_{0} \longrightarrow w \text { as } k \longrightarrow \infty \text {, for some } w \in X
$$

We claim:

$\omega\left(x_{0}\right) \subset \mathcal{E}$ attracts $Y$, and for every $x \in Y$ we have $\Phi_{t_{1}} x \longrightarrow w$ as $k \longrightarrow \infty$. Proof: Set

$$
Y^{*}=\left\{y \in Y: \omega(y)=\omega\left(x_{0}\right) \text { and } \Phi_{t_{1}} y \longrightarrow w \text { as } k \longrightarrow \infty\right\} .
$$

Similarly as in (a) it suffices to show that $Y^{*}$ is both open and closed in $Y$. This is easier when we first show that $Y^{*}$ is order-convex in $X$. Pick any $x, y \in Y^{*}, x<y$. Then for every $z \in[x, y]$ we have $\omega(x) \leq \omega(z) \leq \omega(y)$ whence $\omega(z)=\omega\left(x_{0}\right)$, and $\Phi_{\ell_{k}} z \longrightarrow w$ as $k \longrightarrow \infty$. Hence $z \in Y^{*}$, and $Y^{*}$ is order-convex, by the order-connectedness of $X$.

$Y^{*}$ is open in $\hat{X}$ : Pick any $y \in Y^{*}$. Choose $J$ as in the proof of (a). Then $\omega(a)=$ $\omega(b)=\omega(y)=\omega\left(x_{0}\right)$, and so $a, b \in Y^{*}$. Hence $[a, b] \subset Y^{\bullet}$. Again, we find $u, v \in X$ such that $u \ll y \ll v$ and $\Phi_{1} a \leq \Phi_{1} u \ll \Phi_{1} v \leq \Phi_{1} b$. We conclude that $\omega(u)=\omega(v)=\omega\left(x_{0}\right)$ and $\Phi_{t_{k}} x \longrightarrow w$ as $k \longrightarrow \infty$, for $x \in[u, v]$. In particular, we have $\left.\left.\| u, v\right]\right] \subset Y^{*}$ since $X$ is order-connected. 
$Y^{*}$ is closed in $Y$ : Take any $y \in \mathrm{Cl}\left(Y^{*}\right) \cap Y$. Again, we find $u, v \in X$ such that $u \ll y \ll v$ and $\omega(u)=\omega(v)=\omega(y)$. Choosing any $y^{\prime} \in Y^{*} \cap\{[u, v] \| \neq$ we arrive at $\omega(y)=\omega\left(y^{\prime}\right)=\omega\left(x_{0}\right)$ and all $\Phi_{t_{k}} y^{\prime}, \Phi_{t_{k}} u, \Phi_{t_{k}} v$ and $\Phi_{t_{k}} y$ converge to $w$ as $k \longrightarrow \infty$. Thus $y \in Y^{\bullet}$.

We have proved $Y^{\bullet}=Y$, and $Y$ is order-convex and order-open. In particular, $\omega\left(x_{0}\right) \subset$ $\mathcal{E}$ by Prop. 3.3(b). The remaining part of the proof of (b) is an almost word-by-word repetition of the proof of (a) upon replacing $P^{*}$ by $\omega\left(x_{0}\right)$, and $\Phi_{t}\left(t \in R_{+}^{1}\right)$ by $\Phi_{t_{k}}(k \in N)$, resp. We have completed the proof of our theorem.

Under a mild additional hypothesis on a given component $Y$ of $\mathcal{N}$, called order-quasiboundedness, the order structure of $Y$ and $\partial Y$ is similar to that of a component of $\mathcal{M}$ and its boundary in Lemma 4.3. A set $Y \subset X$ is called order-quasibounded if for every $x \in Y$ there exist $a, b \in X$ such that $a \leq x \leq b$ and $[[-\infty, a] \cap Y \subset\{a\}$ and $[b, \infty]] \cap Y \subset\{b\}$. It is easy to see that every order-bounded set is also order-quasibounded. On the other hand, let $X=V$ and $\Phi: \mathbf{R}_{+}^{1} \times V \longrightarrow V$ satisfy $(V)$ and $(\Phi)$, and let $\Gamma$ be an invariant order resolution of $V$. Consider any $\left(A_{i}, B_{i}\right) \in \Gamma, i=1,2$, such that $A_{1} \subset A_{2}$. Then the set $A_{2} \cap B_{1}$ (and any of its subsets) is order-quasibounded, but $A_{2} \cap B_{1}$ is not order-bounded.

Corollary 4.7. Let $X, P h i$ and $\Gamma$ be as in Lemma 4.3. Assume that every $x \in X$ has relatively compact orbit. Let $Y$ be a component of $\mathcal{N}$ such that $Y$ is order-quasibounded and $\Phi$ is $\omega$-compact in every simply ordered, compact arc $J \subset Y \cup \partial_{-} Y \cup \partial_{+} Y$. Then there exist $\left(A_{1}, B_{1}\right),\left(A_{2}, B_{2}\right) \in \Gamma$ such that $A_{1} \subset A_{2}$ and $Y$ is a component of $\operatorname{Int}\left(A_{2}\right) \cap \operatorname{Int}\left(B_{1}\right)$. In particular, also the statements (a), (b) and (c) from Lemma 4.3 are valid.

Remark. Observe that in this corollary we have dropped the separability hypothesis on every simply ordered, order-connected subset of $\mathcal{E}$ required in Theorem 4.6. Therefore, at this moment we know only that the set $P^{*}$ defined in the proof of (a) in Theorem 4.6 is simply ordered and order-connected. However, when $Y$ is order-quasibounded, the path $P$ in (a) still exists, as it follows from our proof of Corollary 4.7 below.

P ROOF of Corollary 4.7. Let us fix an arbitrary $x_{0} \in Y$. Since $Y$ is order-quasibounded and $X$ is order-connected, $x_{0}$ is contained in a simply ordered, compact arc $J \subset X$ with endpoints $a<b$ having the following property:

If $x \in X$, and either $x<a$ or $x>b$, then $x \notin Y$.

By Theorem 4.6, the set $Y$ is order-convex and order-open, and hence, the set $J^{\prime}=$ $J \cap\left(Y \cup \partial_{-} Y \cup \partial_{+} Y\right)$ is again a compact arc with endpoints $a^{\prime} \in \partial_{-} Y$ and $b^{\prime} \in \partial_{+} Y$ satisfying $J^{\prime} \backslash\left\{a^{\prime}, b^{\prime}\right\} \subset Y^{\prime}$. Consequently, without any loss of generality we may assume that $a \in \partial_{-} Y, b \in \partial_{+} Y$ and $x_{0} \in J^{0}=J \backslash\{a, b\} \subset Y$. Observe that $a, b \notin \mathcal{M}$. Thus, the 
sets

$$
\Gamma^{1}=\{(A, B) \in \Gamma: a \in H=A \cap B\} \text { and } \Gamma^{2}=\{(A, B) \in \Gamma: b \in H=A \cap B\}
$$

are nonempty, disjoint and $A^{1} \subset A^{2}$ whenever $\left(A^{i}, B^{i}\right) \in \Gamma^{i}, i=1,2$. We define

$$
\begin{aligned}
& A_{1}^{\prime}=\cap\left\{A:(A, B) \in \Gamma^{1}\right\}, \quad \text { and } \quad B_{1}^{\prime}=\cup\left\{\operatorname{Int}(B):(A, B) \in \Gamma^{1}\right\}, \\
& A_{2}^{\prime}=\cup\left\{\operatorname{Int}(A):(A, B) \in \Gamma^{2}\right\}, \quad \text { and } \quad B_{2}^{\prime}=\cap\left\{B:(A, B) \in \Gamma^{2}\right\}
\end{aligned}
$$

and set

$$
A_{1}=A_{1}^{\prime}, B_{1}=\mathrm{Cl}\left(B_{1}^{\prime}\right), A_{2}=\mathrm{Cl}\left(A_{2}^{\prime}\right), B_{2}=B_{2}^{\prime}
$$

and $W=\operatorname{Int}\left(A_{2}\right) \cap \operatorname{Int}\left(B_{1}\right)=A_{2}^{\prime} \cap B_{1}^{\prime}$. Using the same arguments as in the proof Theorem 2.2 we deduce that $\left(A_{i}, B_{i}\right) \in \Gamma^{i}$ and $A_{1} \subset A^{1} \subset A^{2} \subset A_{2}$ whenever $\left(A^{i}, B^{i}\right) \in$ for $i=1,2$. It is obvious that $J^{0}$ is contained in a component $W^{\prime}$ of $W$. Since $X$ is ord connected and $W$ is order-convex and order-open, also $W^{\prime}$ is order-convex and order-opi Note that $J^{0} \subset Y \cap W^{\prime}$. We apply Theorem 4.6(a,b) to conclude that $J$ is attracted either an $\omega$-limit fibre $F$ of $J$ in which case $F \subset P^{*}$ as in the proof of Theorem 4.6(a), else to $\omega\left(x_{0}\right)$ as in the proof of Theorem 4.6(b). We set $K=F$ if (a) holds, and $K=\omega(\alpha$ if (b) holds. We claim that

$K$ attracts $W^{\prime}$. Proof: Denote $W^{*}=\left\{x \in W^{\prime}: \omega(x) \subset K\right\}$. Since $J^{0} \subset W^{*}$ and $W^{\prime}$ connected, it suffices to prove that $W^{*}$ is both open and closed in $W^{\prime}$. Similarly as in t proof of Theorem 4.2(b) one first shows that $W^{*}$ is lower and upper closed in $W^{\prime}$, and th. $W^{*}$ is open and closed in $W^{\prime}$. We leave the details to the reader. Now we can prove

$K$ attracts $W^{\prime} \cup \partial_{-} W^{\prime} \cup \partial_{+} W^{\prime}$. Proof: Consider any $x \in \partial_{-} W^{\prime}$; the case $x \in \partial_{+} W^{\prime}$ analogous. Then $\omega(x) \leq \omega(y) \subset K$ whenever $x<y \in W^{\prime}$, and $\omega(x) \subset H_{1}$. Distinguishi: between the cases $K=F$ in which case $F$ is a simply ordered, compact arc with endpoin in $H_{1}$ and $H_{2}$, and $K=\omega\left(x_{0}\right)$ in which case $\omega\left(x_{0}\right) \subset H_{1} \cap H_{2}$, we arrive at $\omega(x)=K \cap H$

In particular, we conclude that $\Phi$ is $\omega$-compact in $W^{\prime} \cup \partial_{-} W^{\prime} \cup \partial_{+} W^{\prime}$, and $W^{\prime} \subset \mathcal{S}$ $\mathcal{S}_{-} \cap \mathcal{S}_{+}, \partial_{-} W^{\prime} \subset \mathcal{S}_{+}, \partial_{+} W^{\prime} \subset \mathcal{S}_{-}$. Hence, $W^{\prime}$ is a connected subset of $\mathcal{N}=\operatorname{Arcint}(X \backslash \mathcal{M}$ and therefore $W^{\prime} \subset Y$. We claim also

$Y \subset W^{\prime}$. Proof: Suppose not, i.e., there is $y_{0} \in Y \backslash W^{\prime}$. Similarly to $x_{0} \in W^{\prime}$, also $y_{0}$ contained in a component $U^{\prime}$ of the set $U=\operatorname{Int}\left(C_{2}\right) \cap \operatorname{Int}\left(D_{1}\right)$ where $\left(C_{i}, D_{i}\right) \in \Gamma, i=1$, are defined for $y_{0}$ exactly as $\left(A_{i}, B_{i}\right)$ for $x_{0}$. By our definition of $J, a, b$ and $\left(A_{i}, B_{i}\right)$ for $x$ we must have $a \in C_{1}$ and $b \in D_{2}$ whence $A_{1} \subset C_{1} \subset C_{2} \subset A_{2}$. It follows that $U^{\prime}$ satisfi not only $U^{\prime} \subset Y$, but also $U^{\prime} \subset W$. Thus, we have shown that $W^{\prime} \subset Y \subset W$ which forc $Y=W^{\prime}$, because $W^{\prime}$ is a component of $W$, and $Y$ is connected. So $Y \subset W^{\prime}$ must hold.

We have proved that $Y$ is a component of $W=\operatorname{Int}\left(A_{2}\right) \cap \operatorname{Int}\left(B_{1}\right)$ as desired. Now $u$ can prove (a), (b) and (c) from Lemma 4.3:

(a) The same as in Lemma 4.3(a).

(b) We have $\partial Y \subset X \backslash(\mathcal{M} \cup \mathcal{N})$. Since $X$ is locally connected, so is $W$. Henc $\partial Y \subset \partial W=H_{1} \cup H_{2}$ because $Y$ is a component of $W$. Similarly as in Lemma 4.3(b) w 
conclude that $\partial_{-} Y$ is an open subset of $\hat{H}_{1}$, and $\partial_{+} Y$ is an open subset of $\hat{H}_{2}$, whereas $\operatorname{Fr}(Y)=\partial Y \cap H_{1} \cap H_{2}$ is closed in $X$.

(c) The sets $Y$ and $X \backslash Y$ are invariant by Theorem 4.6. The invariance of $\partial_{-} Y, \partial_{+} Y$ and $\operatorname{Fr}(Y)$ is proved exactly as in Lemma 4.3(c). This completes the proof.

Our last result in this section is a consequence of Theorems 4.2 and 4.6. It provides valuable information about the domain of attraction of the $\omega$-limit sets and fibres obtained in Theorems 4.2 and $4.6(\mathrm{a}, \mathrm{b})$ :

Theorem 4.8. Let $X$ and $\Phi$ satisfy $(X)$ and $(\Phi)$, and let $X$ be locally connected, strongly ordered and order-connected. Assume that $\Phi$ is $\omega$-compact in every simply ordered, compact arc $J \subset X$. Define $K \subset X$ by one of the following three alternatives:

(i) $K=\{p\}$, for some $p \in \mathcal{E} \cap \mathcal{A}_{1 / 2}$;

(ii) $K=P^{*}$, where $P^{*}$ is a maximal subset of $\mathcal{E}$ with the following property: $P^{*}$ is the image of a strictly increasing continuous path $P: I \longrightarrow X$ defined in a ncndegenerate interval $I \subset R^{1}$;

(iii) $K=\omega\left(x_{0}\right)$, for some $x_{0} \in \mathcal{A}_{1 / 2}$ such that $\omega\left(x_{0}\right) \subset \mathcal{E} \cap\left(\mathcal{U}_{2} \cup \partial_{-} \mathcal{U} \cup \partial_{+} \mathcal{U}\right)$.

Finally, let $\Gamma$ be any invariant order resolution of $X$. Then there exist $\left(A_{1}, B_{1}\right)$, $\left(A_{2}, B_{2}\right) \in \Gamma \cup\{(\emptyset, X),(X, \emptyset)\}$ such that $A_{1} \subset A_{2}, \mathcal{D}(K) \subset A_{2} \cap B_{1}$ and $\operatorname{Int}(\mathcal{D}(K))$ is a nonempty union of some of the components of the set $W=\operatorname{Int}\left(A_{2}\right) \cap \operatorname{Int}\left(B_{1}\right)$. In particular, $\mathcal{D}(K)$ is order-convex, $\partial_{-} \mathcal{D}(K) \subset H_{1}, \partial_{+} \mathcal{D}(K) \subset H_{2}$ and $\operatorname{Fr}(\mathcal{D}(K)) \subset H_{1} \cap H_{2}$, where $H_{i}=A_{i} \cap B_{i}, i=1,2$. Furthermore, for each of the three alternatives above, respectively, we have:

Alt. (i): $p \in \mathcal{A} \Longrightarrow K \subset W, p \in \mathcal{A}_{+} \backslash \mathcal{A}_{-} \Longrightarrow K \subset \partial_{-} W$, and $p \in \mathcal{A}_{-} \backslash \mathcal{A}_{+} \Longrightarrow K \subset$ $\partial_{+} W$; in all these cases $\operatorname{Int}(\mathcal{D}(K))$ is connected.

Alt. (ii): $K \subset W \cup \partial_{-} W \cup \partial_{+} W$; the set $\operatorname{Int}(\mathcal{D}(K))$ is connected.

Alt. (iii): $K \subset \operatorname{Fr}(W)$.

Proof. Since $a \leq x \leq b$ in $X$ implies $\omega(a) \leq \omega(x) \leq \omega(b)$, we obtain that $\mathcal{D}(K)$ is order-convex in $X$, cf. Takác $[43$, Thm. 1.3] or [45, Theorem $2.5(\mathrm{a})]$ for Alt.(ii). It is easy to deduce from Theorems 4.2 and $4.6(\mathrm{a}, \mathrm{b})$ that there exist $a, b \in \mathcal{D}(K)$ satisfying $a \ll b$. Consequently, $[a, b] \subset \mathcal{D}(K)$ entails $\operatorname{Int}(\mathcal{D}(K)) \neq \emptyset$. Next we denote $\tilde{\Gamma}=\Gamma \cup\{(\theta, X),(X, \theta)\}$ and

$$
\Gamma_{K}=\{(A, B) \in \Gamma: A \cap B \cap \mathcal{D}(K) \neq \emptyset\} \text {. }
$$

Observe that our choice of $K$ combined with Def. 2.1 imply $K \subset(X \backslash \mathcal{M}) \cap \mathcal{D}(K)$ where $X \backslash \mathcal{M}=\cup\{H=A \cap B:(A, B) \in \Gamma\}$. In particular, $\Gamma_{K} \neq \emptyset$, and so we may define

$$
C_{1}=\cap\left\{A:(A, B) \in \Gamma_{K}\right\}
$$




$$
\begin{array}{ll}
D_{1}^{\prime}=\cup\left\{\operatorname{Int}(B):(A, B) \in \Gamma_{K}\right\}, & D_{1}=\operatorname{Cl}\left(D_{1}^{\prime}\right) \\
C_{2}^{\prime}=\cup\left\{\operatorname{Int}(A):(A, B) \in \Gamma_{K}\right\}, & C_{2}=\operatorname{Cl}\left(C_{2}^{\prime}\right) \\
D_{2}=\cap\left\{B:(A, B) \in \Gamma_{K}\right\} &
\end{array}
$$

and set $U=\operatorname{Int}\left(C_{2}\right) \cap \operatorname{Int}\left(D_{1}\right)=C_{2}^{\prime} \cap D_{1}^{\prime}$ and $\bar{U}=C_{2} \cap D_{1}$. Using the same arguments as in the proof of Theorem 2.2 we arrive at $\left(C_{i}, D_{i}\right) \in \tilde{\Gamma}, i=1,2$. It is obvious that $K \subset(X \backslash \mathcal{M}) \cap \mathcal{D}(K) \subset \bar{U}$.

As far as $\mathcal{M} \cap \mathcal{D}(K)$ is concerned, we combine Theorem 4.2 with Lemma 4.3 to obtain the following results for each of the alternatives (i), (ii) and (iii), resp.:

Alt. (i): There exist $\left(C_{11}, D_{11}\right),\left(C_{12}, D_{12}\right) \in \tilde{\Gamma}$ such that $C_{11} \subset C_{12} \subset C_{1}$ and

$$
\mathcal{M}_{+} \cap \mathcal{D}(K) \subset U_{1}=\operatorname{Int}\left(C_{12}\right) \cap \operatorname{Int}\left(D_{11}\right) \subset \mathcal{M}
$$

and similarly, there exist $\left(C_{21}, D_{21}\right),\left(C_{22}, D_{22}\right) \in \tilde{\Gamma}$ such that $C_{2} \subset C_{21} \subset C_{22}$ and

$$
\mathcal{M}_{-} \cap \mathcal{D}(K) \subset U_{2}=\operatorname{Int}\left(C_{22}\right) \cap \operatorname{Int}\left(D_{21}\right) \subset \mathcal{M}
$$

More precisely, if $p \in \mathcal{A}=\mathcal{A}_{-} \cap \mathcal{A}_{+}$then $\mathcal{M}_{+} \cap \mathcal{D}(K) \neq \emptyset$ and $\mathcal{M}_{-} \cap \mathcal{D}(K) \neq \emptyset$, and consequently $K=\{p\} \subset \partial_{+} U_{1} \cap \partial_{-} U_{2}$. If $p \in \mathcal{A}_{+} \backslash \mathcal{A}_{-}$then $\mathcal{M}_{+} \cap \mathcal{D}(K)=\emptyset$ (we take $\left.C_{11}=C_{12}=C_{1}\right)$ and $\mathcal{M}_{-} \cap \mathcal{D}(K) \neq 0$, and consequently $K \subset \partial_{-} U_{2}$. The case $p \in \mathcal{A}_{-} \backslash \mathcal{A}_{+}$ is analogous. Thus, in all these cases we have $\left(C_{12}, D_{12}\right),\left(C_{21}, D_{21}\right) \in \Gamma_{K}$ which forces $C_{12}=C_{1}$ and $C_{21}=C_{2}$. We set $\left(A_{i}, B_{i}\right)=\left(C_{i i}, D_{i i}\right)$ for $i=1,2$.

Alt. (ii): If $P^{*}$ is an open arc, then $\mathcal{M} \cap \mathcal{D}(K)=D$, and we set $\left(C_{i j}, D_{i j}\right)=$ $\left(C_{i}, D_{i}\right), i, j=1,2$. If $P^{*}$ possesses a minimal and/or a maximal element $c$ and $d$, resp., we can handle it in the same way as $p$ in Alt (i). For instance, if $\mathcal{M}_{+} \cap \mathcal{D}(K) \neq 0$ then $c \in P^{*}$ and $\mathcal{M}_{+} \cap \mathcal{D}(K)=\mathcal{M}_{+} \cap \mathcal{D}(c)$, where $c \in \mathcal{A}_{-} \backslash \mathcal{A}_{+}$, and we choose $\left(C_{1 i}, D_{1 i}\right)$ exactly as in Alt. (i) with $C_{12}=C_{1}$ as a consequence. In any case, we set $\left(A_{i}, B_{i}\right)=\left(C_{i i}, D_{i i}\right)$ again.

Alt. (iii): We must have $\mathcal{M} \cap \mathcal{D}(K)=0$ since $\omega\left(x_{0}\right) \subset X \backslash \mathcal{A}_{1 / 2}$. We set $\left(C_{i j}, D_{i j}\right)=$ $\left(C_{i}, D_{i}\right)$ for $i, j=1,2$, and $\left(A_{i}, B_{i}\right)=\left(C_{i}, D_{i}\right)$.

For all alternatives (i), (ii) and (iii) we obtain $\mathcal{D}(K) \subset \bar{W}=A_{2} \cap B_{1}$ as desired. Now consider any component $Y$ of $W$ such that $Y^{*}=Y \cap \mathcal{D}(K) \neq 0$. We claim that $Y^{*}=Y$. It suffices to show that $Y^{*}$ is open and closed in $Y$. Similarly as in the proof of Theorem 4.2(b) we only need to prove that $Y^{*}$ is both lower and upper closed in $Y$. Both proofs being analogous, we prove only

$Y^{*}$ is lower closed in $Y$. Proof: Fix any $x \in Y$ and $y \in Y^{*}$ such that $x<y$. Hence, $\omega(x) \leq \omega(y) \subset K$ and $\omega(x) \subset \bar{W}=A_{2} \cap B_{1}$. Suppose $\omega(x) \not \subset K$; hence $\omega(x) \ll \omega(y)$ by Prop. 3.3, and also $\omega(x) \cap K=\emptyset$ by our choice of $K$. It is obvious that $x \notin U=C_{2}^{\prime} \cap D_{1}^{\prime}$, see our definition of $\Gamma_{K}$. So we must have $x \in C_{1}$. Thus, $\omega(x) \subset C_{1}$ together with $K \subset$ $\bar{U}=C_{2} \cap D_{1}$. But also $x \in W=\operatorname{Int}\left(C_{22}\right) \cap \operatorname{Int}\left(D_{11}\right)$, and therefore $x \in C_{1} \cap \operatorname{Int}\left(D_{11}\right) \neq 0$. This is possible only if $C_{11} \neq C_{1}$. Hence, Alt. (iii) is excluded right away, and in Alt. (ii) the set $P^{*}$ possesses a minimal element $c$. In Alt. (i) we set $c=p$. So in Alt. (i) and (ii) 
we arrive at $c \in \mathcal{E}$ and $\{c\}=K \cap C_{1}$, whence $\omega(x) \subset \operatorname{Int}\left(C_{1}\right)$ by $\omega(x) \ll c \leq \omega(y)$. This

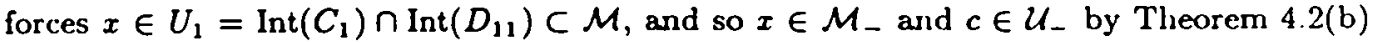
and Lemma 4.3. We conclude that $\mathcal{M}_{+} \cap \mathcal{D}(K)=\mathcal{M}_{+} \cap \mathcal{D}(c)=0$ in which case we have defined $C_{11}=C_{1}$. In particular, $U_{1}=0$ contradicts $x \in U_{1}$, and consequently, also Alt. (i) and (ii) are excluded. We have proved $\omega(x) \subset K$, and so $Y^{\bullet}$ is lower closed in $Y$.

We have verified also $Y=Y^{*} \subset \mathcal{D}(K)$. This implies that $\mathcal{D}(K) \cap W$ is a nonempty union of some of the components of $W$ which, in turn, shows that this union coincides with Int $\left(\mathcal{D}\left(K^{-}\right)\right.$). In particular, we have $\partial_{-} \mathcal{D}(K) \subset H_{1}, \partial_{+} \mathcal{D}(K) \subset H_{2}$ and $\operatorname{Fr}\left(\mathcal{D}\left(K^{-}\right)\right) \subset H_{1} \cap H_{2}$. In Alt. (i) and (ii) we have even $K \subset Y_{0} \cup \partial_{-} Y_{0} \cup \partial_{+} Y_{0}$ where $Y_{0}$ is a component of $\operatorname{Int}(\mathcal{D}(K))$. We need to show $Y_{0}=\operatorname{Int}(\mathcal{D}(K))$. So fix any $x \in \operatorname{Int}(\mathcal{D}(K))$. Observe that $\mathcal{O}^{+}(x)$ is connected, $\mathcal{O}^{+}(x) \subset \operatorname{Int}(\mathcal{D}(K))$ and there exists $\tau \in \mathbf{R}_{+}^{1}$ such that either $\Phi_{\mathrm{r}} x \ll z$ and $p \ll z$ for some $p \in K$ and $z \in Y_{0}$, or else $\Phi_{r} x \gg y$ and $q \gg y$ for some $q \in K$ and $y \in Y_{0}$. Hence, $\Phi_{r} x \in Y_{0}$, which forces $x \in Y_{0}$, i.e., $Y_{0}=\operatorname{Int}(\mathcal{D}(K))$. We have proved that $\operatorname{lnt}(\mathcal{D}(K))$ is connected in Alt (i), (ii). All remaining claims have already been proved.

Remark. Our hypotheses imposed on $X$ in all results of this section, except for Corollary 4.7, are easily satisfied when, for instance, $X$ is a nonempty, order-convex and open subset of a strongly ordered vector space $V$ satisfying $(V)$. If $X=[[a, b]]_{V}$, for some $-\infty \leq a \ll b \leq \infty$, is an open order interval in $V$, then $X$ satisfies also the hypotheses of Corollary 4.7 with $Y$ order-quasibounded, an arbitrary component of $\mathcal{N}=\operatorname{Arcint}(X \backslash \mathcal{M})$, whenever $a, b \in \mathrm{Cl}_{V}(\mathcal{M})$. Observe that $a \in \mathrm{Cl}_{V}(\mathcal{M})$, and analogously for $b$, means that precisely one of the following three alternatives must occur:

(1) There exists $b_{0} \in V$, $a \ll b_{0} \leq b$, such that, for all $x \in\left[\left[a, b_{0}\right]\right]_{v}$ with $\mathrm{Cl}_{V}\left(\mathcal{O}^{+}(x)\right)$ compact, we have $\Phi_{t} x \longrightarrow b_{0}$ in $V$ as $t \longrightarrow \infty$. If $b_{0}=b$ we may set $\Phi_{t} b=b, t \in R_{+}^{1}$, thus extending $\Phi_{t}$ to $X \cup\{b\}$.

(2) There exists $a_{0} \in V$, $a \ll a_{0} \leq b$, such that, for all $x \in\left[\left[a, a_{0}\right]\right] v$ with $\operatorname{Cl}_{V}\left(\mathcal{O}^{+}(x)\right)$ compact, we have $\Phi_{t} x \longrightarrow a$ in $V$ as $t \longrightarrow \infty$. We may set $\Phi_{t} a=a, t \in R_{+}^{1}$.

(3) There exists a sequence $a_{n} \in X \backslash \mathcal{M}$ such that $a_{n+1} \ll a_{n}$ and $\left[\left[a_{n+1}, a_{n}\right]\right] v \cap \mathcal{M} \neq 0$ for each $n \in N$, and $a_{n} \longrightarrow a$ in $V$ as $n \longrightarrow \infty$. Again, we may set $\Phi_{1} a=a, t \in R_{+}^{1}$. If, in addition, $\mathcal{O}^{+}(x)$ is relatively compact in $X$ for each $x \in X$, then we can choose each $a_{n} \in \mathcal{E}$ by induction on $n$, cf. Theorem 4.2(b).

The case $a=-\infty$ and $a_{n} \leq n a_{1} \ll 0 \in V$ for some $a_{n} \in \mathcal{M}, n \in N$, can be treated similarly as $a \in V$, except for (2) which cannot occur. The case $b=\infty$ is analogous. We write shortly $a, b \in \mathrm{Cl}_{V *}(\mathcal{M})$ if these cases occur, where $V^{\#}=V \cup\{-\infty, \infty\}$ has the topology defined by all open subsets of $V$ and all sets $[[-\infty, d]]_{V} \cup\{-\infty\}$ and $[[c, \infty]]_{V} \cup\{\infty\}$ together with their unions and finite intersections, for $c, d \in V$. Thus, $Y$ is order-quasibounded in $X=[[a, b]]_{V}$ whenever $a, b \in \mathrm{Cl}_{V *}(\mathcal{M})$.

Throughout the remaining part of this section we denote by $d$ a metric for $X$ and by $\hat{d}$ a metric for $\hat{X}$, the space $X$ with the order topology. We assume that $\hat{d}$ is an ordered 
metric: If $u \leq a \leq b \leq v$ in $X$ then $\hat{d}(a, b) \leq \hat{d}(u, v)$. For instance, when $X$ is an open subset in a strongly ordered topological vector space $V$ and $e \in \operatorname{Int}\left(V_{+}\right)$, then the metric $\hat{d}(x, y)=|x-y|_{e}$ derived from the ordered norm $|\cdot|_{e}$ on $\hat{V}$, cf. Section 1 , is an ordered metric for $\hat{X}$.

We say that two points $x, y \in X$ are asymptotic if $\dot{d}\left(\Phi_{t} x, \Phi_{t} y\right) \longrightarrow 0$ as $t \longrightarrow \infty$; shortly $x \sim y$. Clearly $\omega(x)=\omega(y)$ whenever $x, y \in X_{0}$ and $x \sim y$.

Making use of the ordered metric $\hat{d}$ for $\hat{X}$ we can state the following trivial result which, however, is very useful for alternatives (i) and (iii) in Theorem 4.8.

Corollary 4.9. Let $X$ and $\Phi$ satisfy $(X)$ and $(\Phi)$, and let $X$ be strongly ordered. Assume that $x, y \in X$ have relatively compact orbits in $X$ and satisfy $x<y$ and $\omega(x)=\omega(y)$. Then $x \sim y$ and also $\hat{d}\left(\Phi_{t} u, \Phi_{t} v\right) \longrightarrow 0$ as $t \longrightarrow \infty$ uniformly for all $u, v \in[x, y]$.

In particular, if either Alt.(i) or Alt.(iii) occurs in Theorem 4.8 , then we can take any $x, y \in \mathcal{D}(K)$ with $x<y$.

\section{Some convergence results}

Throughout this entire section we assume that $X$ and $\Phi$ satisfy $(X)$ and $(\Phi), X$ is locally connected, strongly ordered and order-connected, and $\Phi$ is $\omega$-compact in every simply ordered, compact arc $J \subset X$. In particular, we have $X=\mathcal{S}_{-} \cup \mathcal{U}_{-}=\mathcal{S}_{+} \cup \mathcal{U}_{+}$, disjoint unions. The set $\mathcal{U}=\mathcal{U}_{-} \cup \mathcal{U}_{+}$of all $\omega$-unstable points was described in Proposition 4.4 and its Corollary 4.5. It turned out that $\mathcal{U}$ is, roughly speaking, by one dimension smaller than $X$.

The main purpose of this section is to investigate the $\omega$-limit sets of $\omega$-semistable points, i.e., $\omega(x)$ for $x \in S_{1 / 2}=\mathcal{S}_{-} \cup \mathcal{S}_{+}$. Namely, very little can be said about $\omega(x)$ when $x \in U_{-} \cap U_{+}$as it is shown by an example of Smale [37]. This example is constructed for competitive systems of autonomous ordinary differential equations; it provides an attractor of codimension one with arbitrary dynamics. A time reversal yields an irreducible cooperative system of autonomous ordinary differential equations with arbitrary dynamics in $u_{2}$.

Now we can state our result which was proved already in Takác [45, Thm. 7.1] with a slightly weaker conclusion.

Theorem 5.1. Let $X$ and $\Phi$ satisfy $(X)$ and $(\Phi)$, and let $X$ be locally connected. strongly ordered and order-connected. Assume that $\Phi$ is $\omega$-compact in every simply ordered, compact arc $J \subset X$. Then, given any $x \in \mathcal{S}_{1 / 2}$, precisely one of the following two alternatives is valid:

(i) $\omega(x) \subset \mathcal{S}_{1 / 2}$ in which case $\omega(x)=\{p\}$ for some $p \in \mathcal{E}$; 
(ii) $\omega(x) \subset \mathcal{U}_{2}$ in which case $\omega(x) \subset \mathcal{E}$ and $x \in \mathcal{A}_{1 / 2}$.

In particular, every $x \in S_{1 / 2}$ is quasiconvergent, i.e., $\omega(x) \subset \mathcal{E}$.

If, in addition, $X$ and $V, X \subset V$, are as in Corollary 4.5, then every nonquasiconvergent point $x \in X$ satisfies $x \in \mathcal{U}_{2}$ where the set $\mathcal{U}_{2}=\mathcal{U}_{-} \cap \mathcal{U}_{+}$has been described in Corollary 4.5; its Gaussian measure is zero if $V$ is a separable strongly ordered Banach space.

ProOF. Fix any $x \in S_{1 / 2}$ and $z \in \omega(x)$. We will inspect the following two alternatives:

(i) $z \in \mathcal{S}_{1 / 2}=\mathcal{S}_{-} \cup \mathcal{S}_{+}$; we will assume $z \in \mathcal{S}_{-}$since the case $z \in \mathcal{S}_{+}$is analogous.

(ii) $z \in \mathcal{U}_{2}=\mathcal{U}_{-} \cap \mathcal{U}_{+}$.

From now on we treat each alternative individually:

Alt. (i): Let $z \in \mathcal{S}_{-}$. By our hypotheses on $X$, there exists a simply ordered, compact arc $J \subset X$ with endpoints $z^{\prime}$ and $z$ such that $z^{\prime}<z$. Since $\omega(x)$ is totally invariant and $\Phi_{1}$ strongly increasing, we may assume that $J$ is ordered by $\ll$, i.e., $a<b$ in $J$ implies $a \ll b$. It is also easy to see that we can choose $z^{\prime}$ so close to $z$ that either

(a) $J \backslash\{z\} \subset \mathcal{S}$, or else

(b) there exists a sequence $y_{0}^{\prime} \ll y_{1}^{\prime} \ll y_{2}^{\prime} \ll \cdots$ in $J \cap \mathcal{U}$ such that $y_{n}^{\prime} \longrightarrow z$.

Case (a): By Prop. 4.4, there are only two possibilities left:

$\left(a_{1}\right) z \in \mathcal{A}_{-}$in which case we can choose $z^{\prime}$ so close to $z$ that $\omega\left(z^{\prime}\right)=\omega(z)$.

$\left(8_{2}\right) z \in \mathcal{S}_{-} \backslash \mathcal{A}_{-}$in which case $J$ has an $\omega$-limit fibre $F$, cf. Lemma 3.8 .

Suppose $\left(\mathrm{a}_{1}\right)$ holds. Since $z^{\prime} \ll z \in \omega(x)$, we can find $t \in \mathbf{R}_{+}^{1}$ such that $z^{\prime} \ll \Phi_{1} x$. Consequently $\omega(z)=\omega\left(z^{\prime}\right) \leq \omega(x)$, and obviously $\omega(z) \subset \omega(x)$. But $\omega(x)$ is unordered forces $\omega\left(z^{\prime}\right)=\omega(z)=\omega(x)$. Next $z^{\prime} \ll z \in \omega\left(z^{\prime}\right)$ shows that $z^{\prime} \ll \Phi, z^{\prime}$ for some $s>0$, i.e., $z^{\prime} \in \mathcal{M}_{+}$. Hence, $\omega\left(z^{\prime}\right)$ is a single equilibrium, by Prop. 3.1. In particular, we obtain $\omega(x)=\omega\left(z^{\prime}\right) \in \mathcal{A}_{-}$and $x \in \mathcal{A}_{-}$.

Suppose $\left(\mathrm{a}_{2}\right)$ holds. The same argument as in $\left(\mathrm{a}_{1}\right)$ yields $\omega\left(z^{\prime}\right) \leq \omega(x)$, and also $\omega(z) \subset \omega(x)$. By Prop. 3.10, the set $\bigcup_{y \in J} \omega(y)=F \subset \mathcal{E}$ is the only $\omega$-limit fibre of $J$. We set $M=\{w \in F: w \leq \omega(x)\}$. Observe that $p^{\prime} \in M$ where $p^{\prime}<p$ are the endpoints of $F$; also $\omega\left(z^{\prime}\right)=p^{\prime}$ and $\omega(z)=p$. The compactness of $\omega(x)$ shows that $q=\max (M)$ exists in $M$. We must have $q=p \in \omega(x)$, since otherwise $q<\omega(x)$ would force $q \doteq \Phi_{1} q \ll \Phi_{1} \omega(x)=\omega(x)$, thus contradicting the maximality of $q$ in $M$. We arrive at $\omega(x)=p$ since $\omega(x)$ is unordered. In particular, we obtain $\omega(x)=p \in \mathcal{S}_{-} \backslash \mathcal{A}_{-}$. Thus, we are done with Case (a).

Case (b): By Prop. 4.4 we have $U \subset \partial_{-} \mathcal{M} \cup \partial_{+} \mathcal{M}$. Hence, given $n \in N$, we can find $y_{n} \in J \cap \mathcal{M}$ such that $y_{n-1}^{\prime} \ll y_{n} \ll y_{n+1}^{\prime}$; hence $y_{n} \longrightarrow z$. Passing to a subsequence of $\left\{y_{n}\right\} \subset J$, if necessary, we may assume $y_{1} \ll y_{2} \ll \cdots \ll z$ and either $\left\{y_{n}: n \in N\right\} \subset \mathcal{M}_{-}$ or else $\left\{y_{n}: n \in N\right\} \subset \mathcal{M}_{+}$. Similarly as in $\left(a_{1}\right)$ and $\left(a_{2}\right)$ we obtain $\omega\left(y_{n}\right) \leq \omega(x), n \in N$, and also $\omega(z) \subset \omega(x)$. Since $z \in \mathcal{S}_{-}$, we have

$$
\omega(z)=\omega_{-}(z)=\cap_{k=1}^{\infty} \mathrm{Cl} \cup_{n=k}^{\infty} \omega\left(y_{n}\right)
$$

by Corollary 3.5. It follows that $\omega(z) \leq \omega(x)$, and also $\omega(z) \subset \omega(x)$. Thus, $\omega(z)=\omega(x)$ because $\omega(x)$ is unordered. Furthermore, each $\omega\left(y_{n}\right)=\left\{p_{n}\right\} \subset \mathcal{E} \cap \mathcal{A}_{1 / 2}$ and $y_{n} \in \mathcal{A}$, by 
Theorem 4.2. Obviously $p_{1} \leq p_{2} \leq \ldots$ is a relatively compact sequence with the limit $p=\omega_{-}(z) \in \mathcal{E} \cap \mathcal{S}_{-}$. We obtain $\omega(x)=p \in \mathcal{S}_{-} \backslash \mathcal{A}_{-}$. We are done also with Case (b).

Alt. (ii): By Alt. (i) we have not only $z \in \mathcal{U}_{2}$, but also $\omega(x) \subset \mathcal{U}_{2}$ since $z \in \omega(x)$ is fixed, but still arbitrary. Finally, $x \in \mathcal{A}_{-}$follows from $x \in \mathcal{S}_{-}$combined with $\omega(y) \leq \omega(x) \subset \mathcal{U}_{2}$, for all $y \in X, y<x$. Hence, $\omega(x) \subset \mathcal{E}$ by Prop. 3.3(b). Our theorem is proved.

Remark. Alternative (ii) in Theorem 5.1 seems to be impossible to investigate on a computer since computer errors will cause the convergence of the iterates $x, \Phi_{r} x, \cdots$, $\Phi_{n} x, \cdots$ to one of the equilibria $p$ and $q$, by Prop. 4.4 combined with Prop. 3.1. Here, $\tau>0$ is a fixed time-step, and $p\left(q\right.$, resp.) attracts the component $Y$ of $\mathcal{M}_{-}\left(Z\right.$ of $\left.\mathcal{M}_{+}\right)$ satisfying $\omega(x) \subset \partial_{+} Y \cap \partial_{-} Z$. Fortunately we know that $\omega(x) \subset \mathcal{E}$. But not only that, even $\omega(x)=\{p\} \subset \mathcal{E}$, for every $x \in S_{1 / 2}$, was proved recently by Smith \& Thieme [42, Prop. 2.2] under the following additional $C^{1}$-differentiability and spectral hypotheses on $\Phi$ :

(D) $X \subset V$ where $(V,\|\cdot\|)$ is a strongly ordered Banach space, and there exists $\tau>0$ such that $\Phi_{+}: X \longrightarrow V$ is continuously Fréchet differentiable on $X$, i.e., there exists a continuous mapping $\Phi_{+}^{\prime}: X \longrightarrow \mathcal{L}_{+}(V)$, valued in the cone of all positive continuous linear operators on $V$, such that

$$
\Phi_{r} x-\Phi_{r} x_{0}=\Phi_{r}^{\prime}\left(x_{0}\right)\left(x-x_{0}\right)+\eta\left(x, x_{0}\right)\left\|x-x_{0}\right\|
$$

for $x, x_{0} \in X$, with $\left\|\eta\left(x, x_{0}\right)\right\| \longrightarrow 0$ as $x \longrightarrow x_{0}$.

( $\Sigma)$ For any $p \in \mathcal{E}$ satisfying $\varrho(p) \equiv \operatorname{spr}\left(\Phi_{r}^{\prime}(p)\right) \geq 1, \varrho(p)$ is a pole of the resolvent of $\Phi_{\tau}^{\prime}(p)$ with finite rank and with geometric multiplicity one, and $\left(\varrho(p) I-\Phi_{\tau}^{\prime}(p)\right) v=0$ for some $v \in \operatorname{Int}\left(V_{+}\right)$. Here $\tau>0$ is as in (D).

Here $\operatorname{spr}(L)$ denotes the spectral radius of $L \in \mathcal{L}(V)$. If an isolated point $\lambda \in \mathrm{C}$ of the spectrum of $L \in \mathcal{L}(V)$ is a pole of the resolvent of $L$ with finite order, we define the rank of $\lambda$ by $m_{r}(\lambda)=\operatorname{dim} \bigcup_{k=1}^{\infty} \operatorname{Kernel}\left((\lambda I-L)^{k}\right)$, and the geometric multiplicity of $\lambda$ by $m_{g}(\lambda)=\operatorname{dim}(\operatorname{Kernel}(\lambda I-L))$; thus $m_{g}(\lambda) \leq m_{r}(\lambda) \leq \infty$.

Observe that $(\Sigma)$ is satisfied if, for instance, for any $p \in \mathcal{E}$ satisfying $\varrho(p) \geq 1, \Phi_{r}^{\prime}(p)$ is compact (i.e., completely continuous) and strongly positive, by the Krein-Rutman theorem (cf. Deimling [10, Thm. 19.3]). This is a standard situation for irreducible cooperative systems of ordinary differential equations and semilinear parabolic partial differential equations, cf. Smith \& Thieme [42, Sec. 3]. Also notice that if $p \in \mathcal{E}$ satisfies $\varrho(p)<1$, then $p \in \mathcal{A}$.

The following result is a direct consequence of our Theorem 5.1 combined with Smith \& Thieme [42, Prop. 2.2]:

Theorem 5.2. Let $X$ be an open and order-convex subset of a strongly ordered Banach space $V$ which is also normally ordered, i.e., $V=\hat{V}$ also topologically. Assume that $\Phi$ 
satisfies ( $\Phi)$ and is $\omega$-compact in every compact subset of $X$. Finally, suppose that both $(D)$ and $(\Sigma)$ hold. Then $\omega(x)$ is a single equilibrium for every $x \in \mathcal{S}_{1 / 2}$. Furthermore, we have

$$
\mathcal{U}_{2}=X \backslash \mathcal{S}_{1 / 2} \subset \mathcal{U}_{-} \cup \mathcal{U}_{+} \subset \partial \mathcal{M} \subset \partial(\operatorname{Int}(\mathcal{A}))
$$

and, in particular, $\operatorname{Int}(\mathcal{S})$ is dense in $X$, cf. Proposition 4.4.

If, in addition, $V$ is separable, then $U_{2} \subset U=U_{-} \cup U_{+}$where both $U_{-}$and $U_{+}$are the unions of at most countably many Lipschitz manifolds of codimension one in $V$, cf. Corollary 4.5 .

The density of $\operatorname{Int}(S)$ in $X$ was proved also in Smith \& Thieme [42, Thm. 2.6] by different methods inspired by the work of Poláciik [32] who found sufficient conditions for semilinear parabolic partial differential equations to have a dense open set of convergent points, i.e., $x \in X$ with $\Phi_{t} x \longrightarrow p \in \mathcal{E}$ as $t \longrightarrow \infty$. Except for our results in this article and those in Hirsch [21] and Polácik [33], the structure of the Lipschitz manifolds forming the sets $U_{-}$and $U_{+}$as well as convergence properties of $\Phi$ on them still remain unknown. The following information about the semiflow $\Phi$ on $\mathcal{U}_{2}$ is easily obtained by combining Theorem 4.2 with Prop. 4.4:

Proposition 5.3. Let $X, \Phi$ and $\Gamma$ be as in Theorem 4.2. Assume that every $x \in X$ hes relatively compact orbit. Fiz any $u \in \mathcal{U}_{2}=\mathcal{U}_{-} \cap \mathcal{U}_{+}$. Then there exist a component $Y$ of $\mathcal{M}_{-}$and a component $Z$ of $\mathcal{M}_{+}$such that $\mathcal{O}^{+}(u) \cup \omega(u) \subset \partial_{+} Y \cap \partial_{-} Z \subset \mathcal{U}_{2}$. Moreover, we have $\partial_{+} Y \subset H_{1}=A_{1} \cap B_{1}$ and $\partial_{-} Z \subset H_{2}=A_{2} \cap B_{2}$ for some $\left(A_{i}, B_{i}\right) \in \Gamma, i=1,2$, with $A_{1} \subset A_{2}$, and $p \ll \omega(u) \ll q$ where $\{p\}=\mathcal{E} \cap \partial_{-} Y \subset \mathcal{A}_{+}$attracts $Y \cup \partial_{-} Y$, and $\{q\}=\mathcal{E} \cap \partial_{+} Z \subset \mathcal{A}_{-}$attracts $Z \cup \partial_{+} Z$. Finally,

$$
H_{1} \cap[p, q] \subset \partial_{+} Y \text { and } H_{2} \cap[p, q] \subset \partial_{-} Z \text {. }
$$

If, in addition, $X$ is an order-convex, open subset of $V$ where $V$ satisfies $(V)$, and if $\Phi_{t}[p, q]$ is relatively compact in $V$ for every $t>0$, then there exist also $p^{\prime} \in \mathcal{E} \cap H_{1}$ and $q^{\prime} \in \mathcal{E} \cap H_{2}$ such that $p \ll p^{\prime} \leq q^{\prime} \ll q$. For any such $p^{\prime}$ and $q^{\prime}$ satisfying also $p^{\prime} \neq q^{\prime}$, we have

$$
\left(\mathcal{O}^{+}(u) \cup \omega(u)\right) \cap\left[p^{\prime}, q^{\prime}\right]=\emptyset
$$

ProOF. All claims follow from Theorem 4.2 and Prop. 4.4, except for the existence of $p^{\prime}$ and $q^{\prime}$ which follows directly from Hirsch [20, Proof of Thm. 10.5] or Takác [43, Lemma 3.1] or [45, Prop. 2.3]. 


\section{REFERENCES:}

[1] ADAMS, R. A.: Sobolev Spaces. New York: Academic Press 1975

(2) ALIKAKOS, N. D., and HESS, P.: On stabilization of discrete monotone dynamical systems. Israel J. Math. 59 (1987), 185 - 194

[3] AlikAKOS, N. D., HESS, P., and MATANO, H.: Discrete order preserving semigroups and stability for periodic parabolic differential equations. J. Differential Equations 82 (1989), 322 - 341.

[4] AMANN, H.: Fixed point equations and nonlinear eigenvalue problems in ordered Banach spaces. SIAM Review 18(4) (1976), 620 - 709.

[5] AMANN, H.: Existence and regularity for semilinear parabolic evolution equations. Ann. Scuola Norm. Sup. Pisa (4)11 (1984), 593 - 676.

[6] AMANN, H.: Global existence for semilinear parabolic systems. J. reine angew. Math. 360 (1985), $47-83$

[7] AMANN, H.: Quasilinear parabolic systems under nonlinear boundary conditions. Arch. Rat. Mech. Anal. 92 (1986), 153 - 192.

[8] ARONSSON, G., and MELlander, I.: A deterministic model in biomathematics. Asymptotic behavior and threshold conditions. Math. Biosci. 49 (1980), 207 - 222.

[9] ARONSZAJN, N.: Differentiability of Lipschitzian mappings between Banach spaces. Studia Math. 57 (1976), $147-190$.

[10] Deimling, K.: Nonlinear Functional Analysis. Berlin - Heidelberg - New York: Springer-Verlag 1985.

(11) FIFE, P. C.: Mathematical Aspects of Reacting and Diffusing Systems (Lect. Notes in Biomath. 28). Berlin - Heidelberg - New York: Springer-Verlag 1979.

[12] Freedman, H. 1.: Deterministic Mathematical Models in Population Ecology. New York: M. Dekker 1980.

[13] HENRY, D.: Geometric Theory of Semilinear Parabiolic Equations (Lect. Notes Math. 840). Berlin - Heidelberg - New York: Springer-Verlag 1981.

[14] HESS, P.: On stabilization of discrete strongly order-preserving semigroups and dynamical processes. In: Proc. of Trends in Semigroup Theory and Applications, Trieste 1987 (Lect. Notes Pure Appl. Math.). New York: M. Dekker 1988.

[15] HETHCOTE, H. W., and YORKE, J. A.: Gonorrhea Transmission Dynamics and Control (Lect. Notes in Biomath. 56). Berlin - Heidelberg - New York: Springer-Verlag 1985.

[16] HIRSCH, M. W.: Systems of differential equations which are competitive or cooperative. I: Limit sets. SIAM J. Math. Anal. 13 (1982), 167 - 179.

[17] HIRSCH, M. W.: Systems of differential equations that are competitive or cooperative. II: Convergence almost everywhere. SIAM J. Math. Anal. 16 (1985), 423 - 439.

[18] HIRSCH, M. W.: Differential equations and convergence almost everywhere in strongly monotone semiflows. Contemp. Math. 17 (1983), 267 - 285.

[19] HiRSCH, M. W.: The dynamical systems approach to differential equations. Bull. Amer. Math Soc. $11(1984), 1-64$.

[20] HIRSCH, M. W.: Stability and convergence in strongly monotone dynamical systems. J. reine angew Math. 383 (1988), 1 - 53. 
[21] HIRSCH, M. W.: Systems of differential equations that are competitive or cooperative. III: Competing species. Nonlinearity 1 (1988), $51-71$.

[22] HIRSCH, M. W.: Systems of differential equations that are competitive or cooperative. IV: Structural stability in threedimensional systems. SIAM J. Math. Anal. 21 (1990), 1225 - 1234.

[23] HIRSCH, M. W.: Systems of differential equations that are competitive or cooperative. V: Convergence in 3-dimensional systems. J. Diff. Equations 80 (1989), $94-106$.

[24] Hirsch, M. W., PUGh, C. C., and ShUB, M.: Invariant Manifolds (Lect. Notes Math. 403). Berlin - Heidelberg - New York: Springer-Verlag 1977.

[25] KUO, H-H.: Gaussian Measures in Banach Spaces (Lect. Notes Math. 463). Berlin - Heidelberg New York: Springer-Verlag 1975.

[26] Ladyzhenskaya, O. A., SOlonNikov, V. A., and UraltSeva, N. N.: Linear and Quasilinear Equations of Parabolic Type (Transl. Math. Mon. Vol. 23). Providence, R.I.: Amer. Math. Soc. 1968

[27] LAJMANOVICH, A, and YORKE, J. A.: A deterministic model for gonorrhea in a nonhomogeneous population. Math. Biosci. 28 (1976), 221 - 236.

[28] MORA, X.: Semilinear parabolic problems define semifiows on $C^{k}$ spaces. Trans. Amer. Math. Soc. 278(1) (1983), 21 - 55.

[.29] OTHMER, H.: The qualitative dynamics of a class of biochemical control circuits. J. Math. Biology 3 (1976), 53 - 78.

[30] PAZY, A.: Semigroups of Linear Operators and Applications to Partial Differential Equations. Berlin - Heidelberg - New York: Springer-Verlag 1983.

[31] PHELPS, R. R.: Gaussian null sets and differentiability of Lipschitz map on Banach spaces. Pacific J. Math. 77(2) (1878), 523 - 531.

[32] POLÁCIK, P.: Convergence in smooth strongly monotone flows defined by semilinear parabolic equations. J. Diff. Equations 79 (1989), $89-110$.

[33] PolÁĆIK, P.: Domains of attraction of equilibria and monotonicity properties of convergent trajectories in parabolic systems admitting strong comparison principle. J. reine angew. Math. 400 (1989), 32 - 56.

[34) Protter, M. H., and Weinberger, H. F.: Maximum Principles in Diffenential Equations. Englewood Cliffs, New Jersey: Prentice-Hall Inc. 1967.

[35] SCHAEFER, H. H.: Topological Vector Spaces. Berlin - Heidelberg - New York: Springer-Verlag 1971 .

[36] SELGRADE, J. F.: Asymptotic behavior of solutions to single loop positive feedback systems. J. Diff. Equations 38 (1980), 80 - 103.

[37] SMALE, S.: On the differential equations of species in competition. J. Math. Biology 3 (1976), 5 7.

[38] SMITH, H. L.: Periodic solutions of periodic competitive and cooperative systems. SIAM J. Math. Anal. 17 (1986), 1289 - 1318.

[39] SMITH, H. L.: Cooperative systems of differential equations with concave nonlinearitics. Nonlin. Anal. 10 (1986), 1037 - 1052.

[40] SMITH, H. L.: Systems of ordinary differential equations which generate an order preserving flow. A survey of results. SIAM Review 30(1) (1988), 87-113. 
(41) SMITH, H. L., and THIEME, H. R.: Quasiconvergence and stability for strongly order preserving semifiows. SIAM J. Math. Anal. 21 (1990), 673 - 692.

[42] SMITH, H. L., and THIEME, H. R.: Convergence for strongly order-preserving semiflows. SIAM J. Math. Anal. (to appear).

[43] TAKÁC, P.: Convergence to equilibrium on invariant d-hypersurfaces for strongly increasing discrete-time semigroups. J. Math. Anal. Appl. 148 (1990), 223 - 244.

[44] TAKA்C, P.: Asymptotic behavior of discrete-time semigroups of sublinear, strongly increasing mappings with applications in biology. Nonlin. Anal. 14 (1990), 35 - 42.

[45] TAKÁC, P.: Domains of attraction of generic $\omega$-limit sets for strongly monotone discrete-time semigroups. J. reine angew. Math. (to appear).

[46] TRIEBEL; H.: Interpolation Theory, Function Spaces, Differential Operators. Amsterdam - New York - Oxford: North-Holland Publ. Co. 1978.

[47] WILDER, R. L.: Topology of Manifolds. Providence, R.I.: Amer. Math. Soc. 1949.

\section{Received 23.01.1990}

Author's address:

Prof. Dr. PETER TAKÁC

Mathematics Department

Vanderbilt University

Nashville, TN 37235, USA

\section{Book reviews}

K. Chadan and P. C. Sabatier: Inverse Problems in Quantum Scattering Theory (Texts and Monographs in Physics). New York - Berlin - u.a.: Springer-Verlag 1989, XXXI + 499 p. , 24 fig.

This monograph describes the theoretical foundations and the actual state of the "classical" theory of inverse problems in quantum theory and the historical development of this field. The content consists of 19 chapters: Some.Results from Scattering Theory. Bound States- Eigenfunction Expansions. The Gel'fand-Levitan-Jost-Kohn Method, Applications of the Gel'fand-Levitan Equation. The Marchenko Method. Examples, Special Classes of Potentials. Nonlocal Separable Interactions. Miscellaneous Approaches to the Inverse Problems at Fixed I, Scattering Amplitudes from Elastic Cross Sections. Potentials from the Scattering Amplitude at Fixed Energy: General Equation and Mathematical Tools, Potentials from the Scattering Amplitude at Fixed Energy: Matrix Methods, Potentials from the Scattering Amplitude of Fixed Energy: Operator Methods. The Three-Dimensional Inverse Problem. Miscellaneous Approaches to Inverse Problems at Fixed Energy, Approximate Methods, Inverse Problems in One Dimension, Problems Connected with Discrete Sprectra. Nu merical Problem. 